\title{
Sedentary behaviour, physical activity, and fitness
}

Citation for published version (APA):

van der Velde, J. H. P. M. (2017). Sedentary behaviour, physical activity, and fitness: associations with cardio-metabolic health. [Doctoral Thesis, Maastricht University]. Maastricht University. https://doi.org/10.26481/dis.20171110jvdv

Document status and date:

Published: 01/01/2017

DOI:

10.26481/dis.20171110jvdv

Document Version:

Publisher's PDF, also known as Version of record

\section{Please check the document version of this publication:}

- A submitted manuscript is the version of the article upon submission and before peer-review. There can be important differences between the submitted version and the official published version of record.

People interested in the research are advised to contact the author for the final version of the publication, or visit the DOI to the publisher's website.

- The final author version and the galley proof are versions of the publication after peer review.

- The final published version features the final layout of the paper including the volume, issue and page numbers.

Link to publication

\footnotetext{
General rights rights.

- You may freely distribute the URL identifying the publication in the public portal. please follow below link for the End User Agreement:

www.umlib.nl/taverne-license

Take down policy

If you believe that this document breaches copyright please contact us at:

repository@maastrichtuniversity.nl

providing details and we will investigate your claim.
}

Copyright and moral rights for the publications made accessible in the public portal are retained by the authors and/or other copyright owners and it is a condition of accessing publications that users recognise and abide by the legal requirements associated with these

- Users may download and print one copy of any publication from the public portal for the purpose of private study or research.

- You may not further distribute the material or use it for any profit-making activity or commercial gain

If the publication is distributed under the terms of Article $25 \mathrm{fa}$ of the Dutch Copyright Act, indicated by the "Taverne" license above, 
Sedentary behaviour, physical activity, and

fitness: associations with cardio-metabolic health

Jeroen H.P.M. van der Velde 
(C) Jeroen H.P.M. van der Velde, Maastricht, 2017

Production: Gildeprint - Enschede

ISBN: 978-94-6233748-0 


\title{
Sedentary behaviour, physical activity, and
}

\section{fitness: associations with cardio-metabolic health}

\author{
PROEFSCHRIFT
}

Ter verkrijging van de graad van doctor aan de Universiteit Maastricht, op gezag van de Rector Magnificus, prof. dr. Rianne M. Letschert, volgens het besluit van het College van Decanen, in het openbaar te verdedigen op vrijdag 10 november 2017 om 14:00 uur

$$
\text { door }
$$

Jeroen Henricus Paulus Maria van der Velde 


\section{Promotores:}

Prof. dr. N.C. Schaper

Prof. dr. H.H.C.M Savelberg

\section{Copromotor:}

Dr. ir. A. Koster

\section{Beoordelingscommissie:}

Prof. dr. ir. M.P. Weijenberg (voorzitter)

Dr. J.B.J. Bussmann (Erasmus universitair medisch centrum, Rotterdam)

Prof. dr. M.K.C. Hesselink

Prof. dr. J.P. Kooman

Prof. dr. ir. M. Visser (VU medisch centrum, Amsterdam) 


\section{Table of contents}

Chapter 1 General introduction 7

Chapter 2 Associations of sedentary behaviour and physical activity with 19 physical function - The Maastricht Study Chapter 3 Sedentary behaviour, physical activity and fitness - The
Maastricht Study

Moderate activity and fitness, not sedentary time, are

Chapter 4 independently associated with cardio-metabolic risk in U.S. adults aged 18-49 - NHANES

Sedentary time and higher intensity physical activity versus

Chapter 5 cardio respiratory fitness; what is more important for cardio77 metabolic health for adults aged 40-75? - The Maastricht Study

Chapter 6 Replacement effects of sedentary time on metabolic outcomes - The Maastricht Study

Chapter 7 Summary and general discussion

Appendices $\quad \mathbf{S}$ amenvatting (NL)

Valorisation addendum

Dankwoord (NL)

Curriculum $\mathbf{V}_{\text {itae }}$ 155 



\section{Chapter 1}

General introduction 


\section{Increase in non-communicable diseases globally}

The prevalence of non-communicable diseases (NCDs), such as cardiovascular disease and type 2 diabetes mellitus (T2DM) has increased significantly over the last decades. NCDs are currently responsible for more deaths than all other causes combined: 38 million deaths per year, as reported by the WHO. ${ }^{1}$ In high income countries, $28 \%$ of these NCD deaths occur before the age of 70, leading to loss of productivity. In addition, the complications and comorbidities of NCDs impose a great burden on patients, health care systems, and society. Therefore, the prevention of NCDs is critical.

In many persons NCDs could be prevented, as several risk factors, such as tobacco use and dietary intake, are modifiable. ${ }^{2}$ Another key modifiable risk factor is lack of physical activity, which is estimated to be the main cause of about one-third of T2DM and coronary heart disease cases. ${ }^{3}$ Therefore, in the prevention of NCDs physical activity is important.

\section{Physical activity}

Physical activity is defined as "any bodily movement produced by skeletal muscles that requires energy expenditure". 4 The first study that established the importance of physical activity in preventing disease was performed already more than sixty years ago by Morris et al. in 1953.5 By comparing bus drivers (who sat all day and had low occupational physical activity) with bus conductors (who walked around in the bus all day and had high occupational physical activity), Morris et al. noticed that bus drivers had higher rates of coronary heart diseases, arguably due to the lack of physical activity. Since this land mark publication, decades of physical activity research have led to a broad attention for lack of physical activity as a risk factor for diseases and mortality.6-8 Nowadays, national physical activity guidelines have been established in order to provide guidance in the prevention of NCDs. Although these guidelines differ between countries, most guidelines state to engage at least 150 minutes per week in moderate to vigorous physical activity (MVPA).,49

MVPA is defined as activities $\geq 3.0$ metabolic equivalents (METs) and includes activities such as brisk walking, cycling, heavy housework, and most sports. A MET is a physiological measure to express the energy expended during a certain activity as a ratio to the energy expended when a person is seated and at rest (=1 MET). ${ }^{10,11}$

Biological mechanisms through which MVPA reduces the risk for NCDs have been studied extensively. These mechanisms include improvements in body composition, enhancements in lipid lipoprotein profiles (e.g. improvements in HDL-cholesterol), reductions in blood pressure and systemic inflammation, and improved glucose metabolism. ${ }^{12-14}$ In addition, regular MVPA promotes vascular, muscular, and 
respiratory adaptations that lead to improved muscle quality, muscular strength and cardiorespiratory fitness (CRF). ${ }^{15}$ In particular for older adults, engaging in muscle strengthening activities is important for maintaining a sufficient level of physical function, i.e. the ability to undertake the physical tasks of everyday living. ${ }^{12,16}$ Deterioration in physical function has been associated with loss of independence, a reduced quality of life, disability, and mortality. ${ }^{17}$

\section{Cardio-respiratory fitness (CRF)}

CRF is defined as "the capacity of the cardiovascular and respiratory systems to supply fuel and oxygen during sustained physical activity". ${ }^{18}$ Thus, it's a reflection of the efficiency of the lungs, heart, vascular system and exercising muscles in the transport and use of nutrients and oxygen. As CRF depends strongly on the amount of MVPA, CRF has often been used as an objective measure for level of physical activity. However, differences in frequency and intensity of engagement in physical activity only partly explain differences in CRF between individuals. An estimated $10-50 \%$ of CRF is explained by other factors than physical activity, including genetic differences, behavioural (such as smoking and overweight), and external elements (such as medical condition). ${ }^{19-21}$ Consequently, level of CRF may differ between individuals with similar patterns of physical activity.

CRF is often determined from an exercise tolerance test performed on a treadmill or cycle ergometer. Prospective studies have demonstrated that CRF is important in the aetiology of the metabolic syndrome, cardiovascular disease, and premature mortality. ${ }^{21,22}$ Although health benefits of MVPA are mediated by increased CRF, MVPA also has acute health effects (without improving CRF), which include improvements in blood pressure, blood lipids, and glucose metabolism. ${ }^{23}$ Consequently, despite being interrelated, MVPA and CRF appear to have distinct health benefits.

\section{Total daily activities}

Even though the aforementioned study of Morris et al. from 1953 reported the beneficial effect of light to moderate physical activities, until recently most research and guidelines were focused on MVPA. However, MVPA only comprises a small part of all daily activities in most individuals. To illustrate, US adults engaged on average 23 minutes per day in MVPA. ${ }^{24}$ European adults engaged slightly more in MVPA, but similar to the Americans, the majority of European adults doesn't meet the 150 minutes per week physical activity guidelines. ${ }^{25}$ 
Physical activity of light intensities, such as standing activities and walking, are considerably more prevalent. The health benefits of light intensity physical activity are markedly less described than that of MVPA. ${ }^{26}$ Recently, the importance of light intensity physical activity has been demonstrated in experimental trials. In sedentary individuals, regular intervals of light intensity activities showed similar or even greater effects on several markers of cardio-metabolic health than one daily interval of MVPA, under similar energy expenditure. ${ }^{27,28}$ Moreover, people spend most time of the day in sedentary behaviour. ${ }^{29-31}$

\section{Sedentary behaviour}

Sedentary behaviour is defined as "any waking behaviour, characterized by a low energy expenditure ( $\leq 1.5 \mathrm{METs}$ ), while being in a sitting or reclining posture". ${ }^{32}$ Examples are TV viewing, driving a car, or working at a computer. Sedentary behaviour has been identified as a risk factor for health, regardless of the amount of MVPA. Even people who meet physical activity guidelines appear to be at increased risk for cardiometabolic complications when being highly sedentary. ${ }^{33}$ Therefore, sedentary behaviour is not merely the absence of MVPA (or lack of physical activity), but should be considered as a distinct behaviour with its own physiological pathways. ${ }^{30,33,34}$ To date, sedentary behaviour has been associated with many disease outcomes: (markers of) cardio-metabolic health, ${ }^{35-38}$ cardiovascular disease, ${ }^{26,37,39,40}$ mortality, ${ }^{26,40,41}$ T2DM,37,40,42 and cancer. ${ }^{43}$

While biological mechanisms through which MVPA affects health have been studied extensively, pathways through which sedentary behaviour affect health are largely unidentified. For instance, whether or not sedentary behaviour is associated with CRF or physical function, independent of MVPA, is largely unknown. Although experimental studies suggest that biological mechanisms of sedentary behaviour involve signalling pathways for lipid and glucose metabolism, the exact mechanisms are unclear. $^{44}$

\section{Measurement of physical activity and sedentary behaviour}

For many years, questionnaires have been used to assess physical activity in large epidemiological studies. Usually, questionnaires collect data on specific or structured types of activities, such as sports participation, running, and cycling. Unfortunately, questionnaires are prone to measurement error as they depend on a participants' recall of past activities. As a result, with questionnaires it is challenging to measure light and moderate intensity activities of short duration that occur as part of everyday tasks, which are difficult to recall. ${ }^{45,46}$ 
The first studies that reported harmful associations of sedentary behaviour, used selfreported time spent watching TV as proximate for sedentary behaviour. For example, time spent watching TV has been associated with weight gain and higher risk for T2DM. ${ }^{47,48}$ However, time spent watching TV comprises only a part of total daily sedentary behaviour. More recent questionnaires that aim to assess sedentary behaviour include other sedentary activities, such as reading, sitting at work or motorized travel. Overall, due to recall and reporting bias, self-reported measures of physical activity and sedentary behaviour have limited validity and reliability. ${ }^{45,46,49,50}$

To overcome the issues of measurement of physical activity and sedentary behaviour based on self-report, accelerometers can be used to objectively measure daily activities. Accelerometers, measure the frequency, duration, and amplitude of acceleration of the body segment to which they are attached. ${ }^{49}$ Commonly they are placed on the hip with an elastic belt, although placement on other sites such as the wrist is becoming more commonplace. These acceleration data are translated into 'counts' summed over a specific time period, typically one minute. More counts per minute indicate higher intensity activity. Commonly, when using hip-worn accelerometers, a score $<100$ counts per minute is considered to be sedentary behaviour, ${ }^{50}$ and MVPA has been defined as a score $>2020$ counts per minute. ${ }^{51}$

Although using cut points for counts per minute provides reasonably accurate estimates of physical activity intensity, it is less accurate in determining posture. For example, standing is often misclassified as sedentary behaviour. ${ }^{52,53}$ Standing should not be considered as a sedentary behaviour as muscle activity during standing is about 2.5 times higher than during sitting. ${ }^{54}$ Posture based accelerometers, such as the ActivPAL, overcome this issue. The ActivPAL is placed directly to the skin on the thigh. By combining position of the thigh with acceleration data, posture is more accurately determined. ${ }^{55}$ For example, a horizontal thigh position indicates a sitting or lying posture and a vertical thigh position without activity indicates a standing posture. Consequently, with posture based accelerometers sedentary behaviour is more accurately measured.

\section{Patterns of sedentary behaviour}

In addition to total sedentary time, the pattern in which sedentary time is accumulated may also be of importance. For example, does a person accumulate sedentary time during the day in a few long periods or in many short periods? Means to express this pattern are the number of interruptions in sedentary time (sedentary breaks), the number of prolonged (e.g. $\geq 30$ minutes) uninterrupted sedentary bouts, and the average duration of a sedentary bout. A sedentary break is defined as an interruption from sedentary time of any duration or with any intensity. In other words, it is a 
transition from a sitting or lying position to an upright position. A sedentary bout is defined is an uninterrupted period of sitting, of any duration. To calculate average bout duration, total sedentary time is divided by the number of sedentary bouts. To illustrate, figure 1.1 shows two examples of patterns of sedentary time. In both examples total sedentary time is similar, in example I fewer sedentary breaks and fewer sedentary bouts are seen compared to example II. In addition, two prolonged sedentary bouts are seen in example I. Example II would be associated with more favourable health outcomes, as several observational and experimental studies have shown that more sedentary breaks, are associated with (markers of) cardio-metabolic health. ${ }^{56,57}$

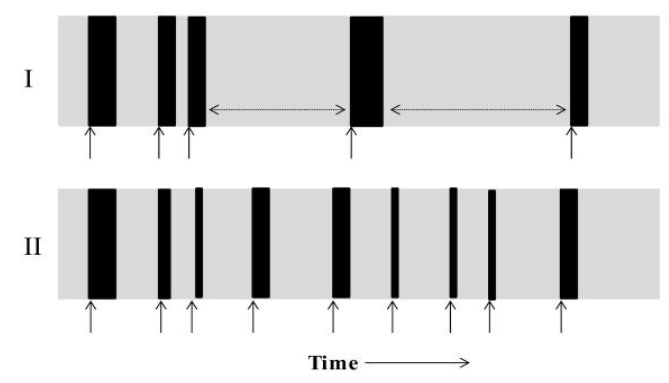

Figure 1.1: Two examples of patterns of sedentary behaviour. Grey bars represent sedentary time, black bars represent non-sedentary time, arrows indicate sedentary breaks, and horizontal arrow lines indicate prolonged sedentary bouts

\section{Aims of this thesis}

As mentioned earlier, engaging in MVPA is important to improve or maintain CRF and physical function. Further, although interrelated, MVPA and CRF are both important in the prevention of cardio-metabolic diseases. Sedentary behaviour has also been associated with cardio-metabolic diseases. However, at this stage it is not clear if the associations between sedentary behaviour and cardio-metabolic health are independent of CRF. In addition, it is uncertain whether or not sedentary behaviour is associated with CRF and physical function. Possibly, if sedentary behaviour is associated with these outcomes, this may aid in understanding the pathways through which sedentary behaviour affects health. Further, a limitation of the majority of physical activity and sedentary behaviour research is the use of self-report to assess daily activities, which has limited validity and reliability. Therefore, the overall aim of this thesis was to improve the insight into sedentary behaviour, physical activity, and CRF and their interrelationship, as risk factors for cardio-metabolic health. These additional insights aid in expanding public health messages and policies aimed at preventing NCDs. We have addressed this aim in two parts. 
In the first part of this thesis we examined the associations of sedentary time and patterns of sedentary behaviour (sedentary breaks etc.) with several measures of physical function, and CRF using a posture based activity monitor (chapters 2 and 3). Importantly, we examined if these associations were independent of the amount of higher intensity physical activity (an approximation of MVPA). In addition, we examined the associations of (theoretically) replacing sedentary time with standing or physical activity on CRF (chapter 3).

In the second part of this thesis we examined the associations of sedentary time, higher intensity physical activity, and CRF with cardio-metabolic health. First, we examined the mutual independent associations of sedentary time, higher intensity physical activity, and CRF with cardio-metabolic health (chapters 4 and 5). Second, the strength of the associations of objectively measured sedentary time, higher intensity physical activity and CRF with several cardio-metabolic risk factors was compared to establish the relative importance of each exposure variable (chapters 4 and 5). Third, we evaluated the combined associations of sedentary time, higher intensity physical activity, and CRF with cardio-metabolic health (chapter 5). These approaches would enable us to provide insight into what is more important for a healthy cardio-metabolic risk profile: the level of CRF or the level of physical activity, either expressed as low amount of sedentary time or high amount of higher intensity physical activity? Last, we examined the associations of (theoretically) replacing sedentary time with standing or physical activity on cardio-metabolic risk factors and the risk for the metabolic syndrome and T2DM (chapter 6). These results provide insight into potential health benefits through reducing sedentary time. The associations investigated in this thesis are illustrated in figure 1.2.

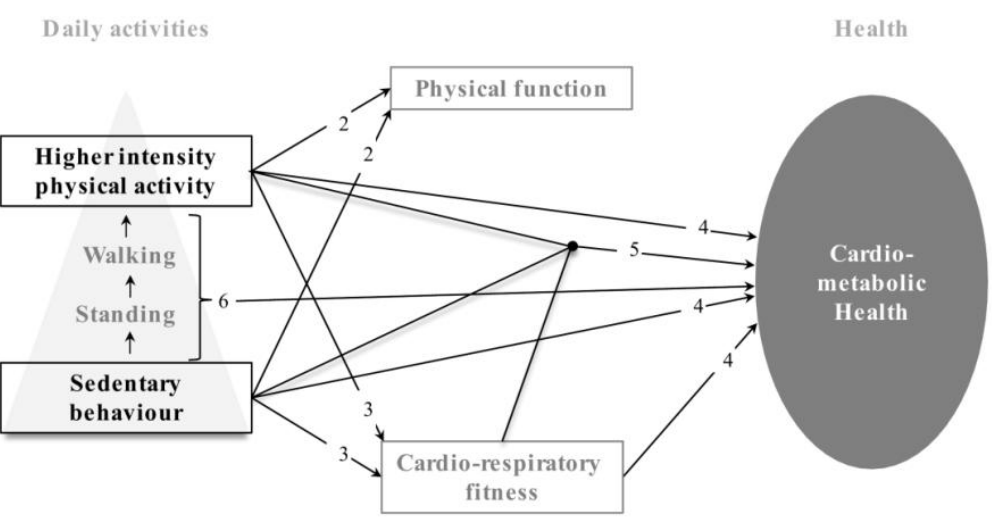

Figure 1.2: Schematic representation of the associations studied in this thesis. Numbers represent corresponding chapters 


\section{Datasets used in this thesis}

In this thesis data were used from two population-based studies: The Maastricht Study and The National Health and Nutrition Examination Survey (NHANES). The Maastricht Study is an ongoing observational population-based cohort study. The rationale and methodology have been described in more detail elsewhere. ${ }^{58}$ In brief, The Maastricht Study focuses on the aetiology, pathophysiology, complications and comorbidities of type 2 diabetes mellitus and is characterized by an extensive phenotyping approach. Cross-sectional data from the first 3,451 participants, who completed the baseline survey between November 2010 and September 2013, was used in this thesis. NHANES is a program of studies designed to assess the health and nutritional status of adults and children in the United States. The purpose of the NHANES initiative is to determine the prevalence of major diseases and risk factors for diseases in the United States. ${ }^{59}$ NHANES data are collected in 2-year cycles using a continuous stratified sampling technique. In this thesis a selection of data from the 2003-2004 cycle was used, as this was the only cycle where data on both objectively measured physical activity and CRF was available. ${ }^{60}$

\section{Outline of this thesis}

In chapter 2 we examined the associations of sedentary time, patterns of sedentary behaviour, and physical activity with measures of physical function and strength. (The Maastricht Study)

In chapter 3 we examined the associations of sedentary time, patterns of sedentary behaviour, and physical activity with cardio-respiratory fitness. (The Maastricht Study)

In chapter 4 we examined the mutually independent associations of sedentary time, physical activity, and cardio-respiratory fitness with cardio-metabolic health in adults aged 18-49 years. (NHANES)

In chapter 5 we examined the independent and combined associations of sedentary time, higher intensity physical activity, and cardio-respiratory fitness with cardiometabolic health, the metabolic syndrome and T2DM. (The Maastricht Study)

In chapter 6 we estimated the associations of the theoretical replacement of sedentary time to standing or physical activity with cardio-metabolic health, the metabolic syndrome and T2DM. (The Maastricht Study)

Chapter 7 provides a discussion of the main findings of this thesis and their implications. Further, this chapter addresses several methodological considerations and proposes directions for future research. 


\section{References}

1. World Health Organization. Global status report on noncommunicable diseases 2014.

2. Perk J, De Backer G, Gohlke H, Graham I, Reiner Ž, Verschuren M, Albus C, Benlian P, Boysen G, Cifkova R. European Guidelines on cardiovascular disease prevention in clinical practice (version 2012). Eur. Heart J. 2012;33(13):1635-1701.

3. World Health Organization \& UNAIDS. Prevention of cardiovascular disease. 2007.

4. World Health Organization. Global recommendations on physical activity for health. 2010.

5. Morris JN, Heady J, Raffle P, Roberts C, Parks J. Coronary heart-disease and physical activity of work. The Lancet 1953;262(6796):1111-1120.

6. Warburton DE, Nicol CW, Bredin SS. Health benefits of physical activity: the evidence. CMAJ 2006;174(6):801-9.

7. Lee I-M, Shiroma EJ, Lobelo F, Puska P, Blair SN, Katzmarzyk PT, Lancet-Physical-Activity-SeriesWorking-Group. Effect of physical inactivity on major non-communicable diseases worldwide: an analysis of burden of disease and life expectancy. The Lancet 2012;380(9838):219-229.

8. Das P, Horton R. Physical activity--time to take it seriously and regularly. The Lancet 2016;388(10051):1254.

9. Kahlmeier S, Wijnhoven TMA, Alpiger P, Schweizer C, Breda J, Martin BW. National physical activity recommendations: systematic overview and analysis of the situation in European countries. BMC Public Health 2015;15(1):1-14.

10. Jette M, Sidney K, Blümchen G. Metabolic equivalents (METS) in exercise testing, exercise prescription, and evaluation of functional capacity. Clin. Cardiol. 1990;13(8):555-565.

11. Ainsworth BE, Haskell WL, Whitt MC, Irwin ML, Swartz AM, Strath SJ, O Brien WL, Bassett DR, Schmitz KH, Emplaincourt PO. Compendium of physical activities: an update of activity codes and MET intensities. Med. Sci. Sports Exerc. 2000;32(9; SUPP/1):S498-S504.

12. Bauman A, Merom D, Bull FC, Buchner DM, Singh MAF. Updating the evidence for physical activity: summative reviews of the epidemiological evidence, prevalence, and interventions to promote "Active Aging". The Gerontologist 2016;56(Suppl 2):S268-S280.

13. Lin X, Zhang X, Guo J, Roberts CK, McKenzie S, Wu WC, Liu S, Song Y. Effects of Exercise Training on Cardiorespiratory Fitness and Biomarkers of Cardiometabolic Health: A Systematic Review and Meta-Analysis of Randomized Controlled Trials. J. Am. Heart. Assoc. 2015;4(7):e002014.

14. Warburton DE, Charlesworth S, Ivey A, Nettlefold L, Bredin SS. A systematic review of the evidence for Canada's Physical Activity Guidelines for Adults. Int. J. Behav. Nutr. Phys. Act. 2010;7(1):1.

15. McArdle WD, Katch FI, Katch VL. Exercise physiology: nutrition, energy, and human performance Lippincott Williams \& Wilkins, 2010.

16. Paterson DH, Warburton DE. Physical activity and functional limitations in older adults: a systematic review related to Canada's Physical Activity Guidelines. Int. J. Behav. Nutr. Phys. Act. 2010;7(38):38.

17. Cooper R, Kuh D, Cooper C, Gale CR, Lawlor DA, Matthews F, Hardy R, Falcon, Teams HAS. Objective measures of physical capability and subsequent health: a systematic review. Age Ageing 2011;40(1):14-23.

18. American College of Sports Medicine. ACSM's guidelines for exercise testing and prescription Lippincott Williams \& Wilkins, 2013.

19. Bouchard C, Rankinen T. Individual differences in response to regular physical activity. Med. Sci. Sports Exerc. 2001;33(6 Suppl):S446-51; discussion S452-3.

20. Gill JM, Celis-Morales CA, Ghouri N. Physical activity, ethnicity and cardio-metabolic health: does one size fit all? Atherosclerosis 2014;232(2):319-333.

21. Lee DC, Artero EG, Sui X, Blair SN. Review: Mortality trends in the general population: the importance of cardiorespiratory fitness. J. Psychopharmacol. 2010;24(4 suppl):27-35.

22. Kodama S, Saito K, Tanaka S, Maki M, Yachi Y, Asumi M, Sugawara A, Totsuka K, Shimano H, Ohashi Y, Yamada N, Sone H. Cardiorespiratory Fitness as a Quantitative Predictor of All-Cause Mortality and Cardiovascular Events in Healthy Men and Women A Meta-analysis. Jama-Journal of the American Medical Association 2009;301(19):2024-2035. 
23. Thompson PD, Crouse SF, Goodpaster B, Kelley D, Moyna N, Pescatello L. The acute versus the chronic response to exercise. Med. Sci. Sports Exerc. 2001;33(6 Suppl):S438-45; discussion S452-3.

24. Schuna JM, Johnson WD, Tudor-Locke C. Adult self-reported and objectively monitored physical activity and sedentary behavior: NHANES 2005-2006. Int. J. Behav. Nutr. Phys. Act. 2013;10(1):126.

25. Marsaux CF, Celis-Morales C, Hoonhout J, Claassen A, Goris A, Forster H, Fallaize R, Macready AL, Navas-Carretero S, Kolossa S. Objectively Measured Physical Activity in European Adults: Cross-Sectional Findings from the Food4Me Study. PLoS ONE 2016;11(3):e0150902.

26. Young DR, Hivert M-F, Alhassan S, Camhi SM, Ferguson JF, Katzmarzyk PT, Lewis CE, Owen N, Perry CK, Siddique J. Sedentary Behavior and Cardiovascular Morbidity and Mortality: A Science Advisory From the American Heart Association. Circulation 2016;134(13):e262-e279.

27. Duvivier BM, Schaper NC, Bremers MA, van Crombrugge G, Menheere PP, Kars M, Savelberg HH. Minimal Intensity Physical Activity (Standing and Walking) of Longer Duration Improves Insulin Action and Plasma Lipids More than Shorter Periods of Moderate to Vigorous Exercise (Cycling) in Sedentary Subjects When Energy Expenditure Is Comparable. PLoS ONE 2013;8(2):e55542.

28. Duvivier BM, Schaper NC, Hesselink MK, van Kan L, Stienen N, Winkens B, Koster A, Savelberg $\mathrm{HH}$. Breaking sitting with light activities vs structured exercise: a randomised crossover study demonstrating benefits for glycaemic control and insulin sensitivity in type 2 diabetes. Diabetologia 2017;60(3):490-498.

29. Matthews CE, Chen KY, Freedson PS, Buchowski MS, Beech BM, Pate RR, Troiano RP. Amount of time spent in sedentary behaviors in the United States, 2003-2004. Am. J. Epidemiol. 2008;167(7):875881.

30. Dunstan DW, Howard B, Healy GN, Owen N. Too much sitting-a health hazard. Diabetes Res. Clin. Pract. 2012;97(3):368-376.

31. Bennie JA, Chau JY, van der Ploeg HP, Stamatakis E, Do A, Bauman A. The prevalence and correlates of sitting in European adults-a comparison of 32 Eurobarometer-participating countries. Int. J. Behav. Nutr. Phys. Act. 2013;10(1):1.

32. Sedentary Behaviour Research Network. (2102). Letter to the editor: standardized use of the terms "sedentary" and "sedentary behaviours". Appl. Physiol. Nutr. Metab 2012;37(3):540-542.

33. Owen N, Healy GN, Matthews CE, Dunstan DW. Too much sitting: the population health science of sedentary behavior. Exerc. Sport Sci. Rev. 2010;38(3):105-13.

34. Hamilton MT, Healy GN, Dunstan DW, Zderic TW, Owen N. Too little exercise and too much sitting: inactivity physiology and the need for new recommendations on sedentary behavior. Curr Cardiovasc Risk Rep 2008;2(4):292-298.

35. Brocklebank LA, Falconer CL, Page AS, Perry R, Cooper AR. Accelerometer-measured sedentary time and cardiometabolic biomarkers: A systematic review. Prev. Med. 2015;76:92-102.

36. Saunders TJ, Larouche R, Colley RC, Tremblay MS. Acute sedentary behaviour and markers of cardiometabolic risk: a systematic review of intervention studies. J Nutr Metab 2012;2012:712435.

37. Rezende LFM, Rey-López JP, Matsudo VKR, Luiz OC. Sedentary behavior and health outcomes among older adults: a systematic review. BMC Public Health 2014;14(1):333.

38. Edwardson CL, Gorely T, Davies MJ, Gray LJ, Khunti K, Wilmot EG, Yates T, Biddle SJ. Association of sedentary behaviour with metabolic syndrome: a meta-analysis. PLoS ONE 2012;7(4):e34916.

39. Ford ES, Caspersen CJ. Sedentary behaviour and cardiovascular disease: a review of prospective studies. Int. J. Epidemiol. 2012;41(5):1338-53.

40. Wilmot EG, Edwardson CL, Achana FA, Davies MJ, Gorely T, Gray LJ, Khunti K, Yates T, Biddle SJ. Sedentary time in adults and the association with diabetes, cardiovascular disease and death: systematic review and meta-analysis. Diabetologia 2012;55(11):2895-2905.

41. Chau JY, Grunseit AC, Chey T, Stamatakis E, Brown WJ, Matthews CE, Bauman AE, van der Ploeg HP. Daily sitting time and all-cause mortality: a meta-analysis. PLoS ONE 2013;8(11):e80000.

42. van der Berg JD, Stehouwer CD, Bosma H, van der Velde JH, Willems PJ, Savelberg HH, Schram MT, Sep SJ, van der Kallen CJ, Henry RM. Associations of total amount and patterns of sedentary behaviour with type 2 diabetes and the metabolic syndrome: The Maastricht Study. Diabetologia 2016;59(4):709-718.

43. Lynch BM. Sedentary behavior and cancer: a systematic review of the literature and proposed biological mechanisms. Cancer Epidemiol. Biomark. Prev. 2010:1055-9965. EPI-10-0815. 
44. Hamilton MT, Hamilton DG, Zderic TW. Role of low energy expenditure and sitting in obesity, metabolic syndrome, type 2 diabetes, and cardiovascular disease. Diabetes 2007;56(11):2655-67.

45. Shephard RJ. Limits to the measurement of habitual physical activity by questionnaires. Br. J. Sports Med. 2003;37(3):197-206.

46. Janz K. Physical activity in epidemiology: moving from questionnaire to objective measurement. Br. J. Sports Med. 2006;40(3):191-192.

47. Tucker LA, Friedman GM. Television viewing and obesity in adult males. Am. J. Public Health 1989;79(4):516-518.

48. Hu FB, Leitzmann MF, Stampfer MJ, Colditz GA, Willett WC, Rimm EB. Physical activity and television watching in relation to risk for type 2 diabetes mellitus in men. Arch. Intern. Med. 2001;161(12):1542-1548.

49. Atkin AJ, Gorely T, Clemes SA, Yates T, Edwardson C, Brage S, Salmon J, Marshall SJ, Biddle SJ. Methods of measurement in epidemiology: sedentary behaviour. Int. J. Epidemiol. 2012;41(5):14601471.

50. Healy GN, Clark BK, Winkler EA, Gardiner PA, Brown WJ, Matthews CE. Measurement of adults' sedentary time in population-based studies. Am. J. Prev. Med. 2011;41(2):216-227.

51. Troiano RP, Berrigan D, Dodd KW, Masse LC, Tilert T, McDowell M. Physical activity in the United States measured by accelerometer. Med. Sci. Sports Exerc. 2008;40(1):181.

52. Bassett DR. Device-based monitoring in physical activity and public health research. Physiol. Meas. 2012;33(11):1769.

53. Schrack JA, Cooper R, Koster A, Shiroma EJ, Murabito JM, Rejeski WJ, Ferrucci L, Harris TB. Assessing daily physical activity in older adults: unraveling the complexity of monitors, measures, and methods. The Journals of Gerontology Series A: Biological Sciences and Medical Sciences 2016:glw026.

54. Tikkanen O, Haakana P, Pesola AJ, Häkkinen K, Rantalainen T, Havu M, Pullinen T, Finni T. Muscle activity and inactivity periods during normal daily life. PLoS ONE 2013;8(1):e52228.

55. Kozey-Keadle S, Libertine A, Lyden K, Staudenmayer J, Freedson PS. Validation of wearable monitors for assessing sedentary behavior. Med. Sci. Sports Exerc. 2011;43(8):1561-7.

56. Chastin SF, Egerton T, Leask C, Stamatakis E. Meta-analysis of the relationship between breaks in sedentary behavior and cardiometabolic health. Obesity 2015;23(9):1800-1810.

57. Benatti FB, Ried-Larsen M. The effects of breaking up prolonged sitting time. Med. Sci. Sports Exerc. 2015.

58. Schram MT, Sep SJ, van der Kallen CJ, Dagnelie PC, Koster A, Schaper N, Henry RM, Stehouwer $\mathrm{CD}$. The Maastricht Study: an extensive phenotyping study on determinants of type 2 diabetes, its complications and its comorbidities. Eur. J. Epidemiol. 2014;29(6):439-51.

59. National Center for Health Statistics. Introduction to NHANES. [cited February 2017] Available from: https://www.cdc.gov/Nchs/Nhanes/about_nhanes.htm

60. National health and nutrition examination survey data NHANES 2003-2004. [cited February 2017] Available from: Http://wwwn.Cdc.Gov/nchs/nhanes/search/nhanes03_04.Aspx. 



\section{Chapter 2}

\section{Associations of sedentary behaviour and physical activity with physical function \\ - The Maastricht Study -}

Jeroen HPM van der Velde, Hans HCM Savelberg, Julianne D van der Berg, Simone JS Sep, Carla JH van der Kallen, Pieter C Dagnelie, Miranda T Schram, Ronald MA Henry, Petronella LM Reijven, Tineke ACM van Geel, Coen DA Stehouwer, Annemarie Koster, Nicolaas C Schaper 


\section{Abstract}

Background: In an ageing population, regular physical activity (PA) and exercise hav been recognized as important factors in maintaining physical function and thereby preventing loss of independence and disability. However, (older) adults spent the majority of their day sedentary and therefore insight into the consequences of sedentary behaviour on physical function, independent of PA, is warranted.

Aim: To examine the associations of objectively measured sedentary time (ST), patterns of sedentary behaviour, overall PA, and higher intensity PA (HPA) with objective measures of physical function.

Methods: Included were 1,932 men and women (aged 40-75 years) participating in The Maastricht Study. The activPAL3 was used to assess daily sedentary behaviour: ST (h), sedentary breaks $(n)$, prolonged $(\geq 30 \mathrm{~min})$ sedentary bouts $(n)$, and to assess time spent in (H)PA (h). Measures of physical function included: covered distance during a six minute walk test (6MWD (meters)), timed chair rise stand test performance (TCSTtime (seconds)), grip strength $\left(\mathrm{kg} \mathrm{kg}^{-1}\right)$, and elbow flexion and knee extension strength $(\mathrm{Nm}$ $\mathrm{kg} 1$ ). Linear regression analyses were used to examine associations between daily sedentary behaviour and PA with physical function.

Results: Every additional hour ST was associated with shorter 6MWD (B = -2.69 m $(95 \% \mathrm{CI}=-4.69 ;-0.69))$ and lower relative elbow extension strength $\left(\mathrm{B}=-0.01 \mathrm{Nm} \mathrm{kg} \mathrm{N}^{-1}\right.$ $(-0.02 ; 0.00)$. More sedentary breaks were associated with faster TCST time: $_{\text {B }}=-0.55 \mathrm{~s}$ $(-0.85 ;-0.26)$. Longer average sedentary bout duration was associated with slower $\mathrm{TCST}_{\text {time }}(\mathrm{B}=0.17 \mathrm{~s}(0.09 ; 0.25))$ and lower knee extension strength $\left(\mathrm{B}=-0.01 \mathrm{Nm} \mathrm{kg}{ }^{-1}\right.$ $(-0.02 ; 0.00))$. Every hour of PA and HPA were associated with greater $6 \mathrm{MWD}\left(\mathrm{B}_{\mathrm{PA}}=\right.$ $\left.15.88 \mathrm{~m}(9.87 ; 21.89), \mathrm{B}_{\mathrm{HPA}}=40.72 \mathrm{~m}(30.18 ; 51.25)\right)$ and faster $\mathrm{TCST}_{\text {time }}\left(\mathrm{B}_{\mathrm{PA}}=-0.55 \mathrm{~s}\right.$ $\left.(-1.03 ;-0.07), \mathrm{B}_{\mathrm{HPA}}=-2.25 \mathrm{~s}(-3.09 ;-1.41)\right)$. HPA was also associated with greater elbow flexion $\left(B=0.05 \mathrm{Nm} \mathrm{kg}^{-1}(0.01 ; 0.08)\right)$ and knee extension strength $\left(B=0.13 \mathrm{Nm} \mathrm{kg}^{-1}\right.$ $(0.06 ; 0.20))$.

Conclusion: In adults aged 40-75 years, sedentary behaviour appeared to be marginally associated with lower physical function, independent of HPA. This suggests that merely reducing sedentary behaviour is insufficient to improve/maintain physical function. In contrast, engaging regularly in PA, in particular HPA, is important for physical function. 


\section{Introduction}

Physical function, or physical capability, can be defined as the degree to which a person can manage the physical tasks of daily living. This can be objectified by several performance tests such as strength, walking speed, and mobility. Deterioration in physical function has been associated with loss of independence, a reduced quality of life, disability, and mortality. ${ }^{1,2}$ Limitations in physical functioning occur more often in later stages of life. For example, in the European Union $27 \%$ of the total population reported limitations in daily activities, for adults aged $>65$ years this was $\sim 40 \%$, and for adults aged $>75$ years in excess of $60 \% .^{3}$ In an ageing population, such as in many European countries, the number of people at risk for functional limitations will increase further. Thus, identifying modifiable determinants that are important for improving or maintaining physical function is imperative.

One of the determinants important for improving or maintaining physical function is physical activity (PA). ${ }^{4}$ Particularly PA of higher intensity, often termed moderate to vigorous PA (MVPA), has been recognized as a major determinant for overall physical wellbeing. ${ }^{5}$ Positive associations of MVPA with physical function ${ }^{4,6}$ and with leg strength ${ }^{7}$ have been reported. The importance of MVPA is nowadays well recognized and worldwide most physical activity guidelines advocate to spend at least 150 minutes/week in MVPA. ${ }^{8}$ However, MVPA only comprises a small part of daily activities as most of the day is generally spent in sedentary behavior in current Westernized societies. ${ }^{9}$ In recent years, there has been a growing interest in sedentary behavior as a determinant for adverse health outcomes.

Sedentary behavior refers to any waking behavior characterized by an energy expenditure $\leq 1.5$ metabolic equivalents (METs) while in a sitting or reclining position. ${ }^{10} \mathrm{An}$ increasing number of studies have associated a larger amount of sedentary time (ST) was associated with unfavorable metabolic and cardiovascular risk markers independent of MVPA. ${ }^{11,12}$ However whether or not a larger amount of ST is associated with lower physical function less clear. Several population based studies have examined the association of ST and physical function. ${ }^{13-18}$ Findings from these studies were inconsistent as some studies have observed an association between larger amounts of ST and worse physical function, ${ }^{13-16}$ whereas other studies have not. ${ }^{17,18}$ Additionally, not only total ST, but also the pattern in which sedentary time is accumulated may be of importance for health. This pattern can be expressed by the number of interruptions in sedentary time (sedentary breaks), by the average duration of uninterrupted periods of sitting or by the number of prolonged (e.g. $\geq 30 \mathrm{~min}$ ) uninterrupted sedentary bouts. In studies with older adults (mean age $>70$ years), more sedentary breaks have been associated with a higher score on the senior fitness test and physical performance tests. ${ }^{19,20}$ 
Whether or not these patterns are associated with physical function in younger adults is uncertain.

As sedentary behavior appears to increase with age, ${ }^{21,22}$ an improved insight into the associations of ST (and the pattern in which this is accumulated) with physical function is warranted. If such associations exist, reducing sedentary behavior could be important in the prevention of functional limitations. Therefore, our objective was to examine the associations of objectively measured ST, patterns of sedentary behavior, overall PA, and higher intensity PA (HPA) with objective measures of physical function in an adult population aged $40-75$ years.

\section{Methods}

\section{Population}

We used data from The Maastricht Study, an observational prospective populationbased cohort study. The rationale and methodology have been described previously. ${ }^{23}$ In brief, the study focuses on the etiology, pathophysiology, complications and comorbidities of type 2 diabetes mellitus (T2DM) and is characterized by an extensive phenotyping approach. Eligible for participation were all individuals aged between 40 and 75 years and living in the southern part of the Netherlands. Participants were recruited through mass media campaigns and from the municipal registries and the regional Diabetes Patient Registry via mailings. Recruitment was stratified according to known T2DM status, with an oversampling of individuals with T2DM, for reasons of efficiency. The present report includes cross-sectional data from a convenience sample of the first 3,451 participants, who completed the baseline survey between November 2010 and September 2013. Data were available for 1,932 participants, after excluding participants that did not receive an accelerometer due to logistics $(n=673)$, with invalid accelerometer readings $(n=136)$, with missing/unperformed physical function testing $(n=629)$ or with missing covariates $(n=81)$. The examinations of each participant were performed within a time window of three months. The study has been approved by the institutional medical ethical committee (NL31329.068.10) and the Minister of Health, Welfare and Sports of the Netherlands (Permit 131088-105234-PG). All participants gave written informed consent.

\section{Accelerometry: sedentary behavior, PA and HPA}

Daily activity levels were measured using the activPAL3 ${ }^{\mathrm{TM}}$ physical activity monitor (PAL Technologies, Glasgow, UK). The activPAL3 is a small (53 $\times 35 \times 7 \mathrm{~mm})$, lightweight (15 g) triaxial accelerometer that records movement in the vertical, anteroposterior and mediolateral axes, and also determines posture (sitting or lying, standing and stepping) based on acceleration information. The device was attached directly to the 
skin on the front of the right thigh with transparent $3 \mathrm{M}$ Tegaderm ${ }^{\mathrm{TM}}$ tape, after the device had been waterproofed using a nitrile sleeve. Participants were asked to wear the accelerometer for 8 consecutive days, without removing it at any time. To avoid inaccurately identifying non-wear time, participants were asked not to replace the device once removed. Data were uploaded using the activPAL software and processed using customized software written in MATLAB R2013b (MathWorks, Natick, MA, USA). Data from the first day were excluded from the analysis because participants performed physical function tests at the research center after the device was attached. In addition, data from the final wear day providing $\leq 14$ waking hours of data were excluded from the analysis. Participants were included if they provided at least 1 valid day ( $\geq 10$ h of waking data).

The total amount of sedentary time was based on the sedentary posture (sitting or lying), and calculated as the mean time spent in a sedentary position during waking time per day. The method used to determine waking time has been described elsewhere. ${ }^{24}$ The total amount of standing time was based on the standing posture, and calculated as the mean time spent standing during waking time per day. The total amount of stepping was based on the stepping posture, and calculated as the mean time stepping during waking time per day. Stepping time (physical activity) was further classified into higher intensity physical activity (HPA; minutes with a step frequency $>110$ steps/min during waking time) and lower intensity physical activity (LPA; minutes with a step frequency $\leq 110$ steps/min during waking time). ${ }^{25}$

The number of sedentary breaks during waking time was determined as each transition from a sitting or lying position to standing or stepping with a duration of at least $1 \mathrm{~min}$, and the mean number of breaks per day was calculated. Sedentary time accumulated in a consecutive period $\geq 30$ min was defined as a prolonged sedentary bout, and the mean number of prolonged sedentary bouts during waking time per day was calculated.

\section{Physical function}

Physical function was assessed by four different tests: distance covered during a fast paced six minute walk test (6MWT), the timed chair stand test (TCST), hand grip strength, and isometric strength tests of the knee extensors and elbow flexors.

\section{Six minute walk test}

Participants were excluded from the 6MWT if they had experienced cardiovascular complications in the preceding three months, had severe hypertension (SBP $\geq 180$ and/or $\mathrm{DBP} \geq 110 \mathrm{mmHg}$ ), a resting heart rate of $<40$ of $>110$ beats $\mathrm{min}^{-1}$, used a walker, or had other medical conditions which prevented them from walking independently. In a hallway, two cones were placed 20 meters apart around which the participants had to make turns. Participant were instructed to walk as many laps as possible in 6 minutes 
at a fast pace without running. Standardized encouragement was given every minute during the test. After 6 minutes or when the participant was unwilling or unable to continue, the covered distance was measured. The covered distance (6MWD) in meters was used as measure for analyses.

\section{Timed chair rise stand test}

The timed chair rise stand test (TCST) was performed on a $46 \mathrm{~cm}$ high chair with a straight back and no arm-rests. The test started with the participant in a sitting position with his/her arms crossed over the chest. Participants were instructed to stand up to a full up-right position and to sit down again as quickly as possible, without using their arms or hands to support. The time (in seconds) needed for ten repetitions (TCST time $_{\text {) }}$ was measured to the nearest of one decimal and was used for analyses.

\section{Handgrip strength}

Handgrip strength was measured with the Jamar handheld dynamometer (SEHAN Corp., Korea- Biometrics Europe BV, Almere). During the test the participant was standing straight against the wall with the upper arm along the trunk and the elbow in $90^{\circ}$ flexion. Participants were instructed to squeeze as hard as possible in the dynamometer for 3-5 seconds, while given standard encouragement. The measurement was performed three times on each hand, alternating hands. Maximal strength $(\mathrm{kg})$ from every trial was recorded. Maximum strength (in $\mathrm{kg}$ ) out of all trials was normalized for body mass and was used for analyses.

\section{Isometric muscle strength test}

Isometric muscle strength of the knee extensors and elbow flexors was assessed in a customized set-up with 2 dynamometers (Futek LSB302, FUTEK Advanced Sensor Technology Inc, Irvine, CA, USA) and recorded with the M-PAQ (Maastricht Instruments, Maastricht, the Netherlands). Measurements were performed on the right leg and arm. Participants were (partly) excluded from the test if they had undergone surgery on the right arm or leg in the preceding three months, or reported relevant injuries on the right arm or leg.

Participants were positioned up-right in the chair (hip angle $110^{\circ}$ ) with their knees flexed in a $90^{\circ}$ angle and the upper leg fixated. A strap connected to the dynamometer (with the axis of the dynamometer corresponding to the knee-joint axis) was secured 2 $\mathrm{cm}$ above the lateral malleolus. Participants were instructed to extend their knee as powerful as possible for five seconds. Three trials were performed. For the measurement of elbow flexion strength the participant remained up-right in the chair with the elbow flexed in a $90^{\circ}$ angle. A strap connected to the dynamometer was secured $2 \mathrm{~cm}$ proximally from the wrist (with the axis of the dynamometer corresponding to the elbow-joint axis). Participants were instructed to flex their elbow as powerful as possi- 
ble for five seconds. Three trials were performed. Participants were able to see the force generated on a monitor. During the trials participants were instructed to refrain from compensatory movements.

To calculate joint torques (Nm) for elbow and knee the force applied on the dynamometers $(\mathrm{N})$ was multiplied by the corresponding moment-arm (distance from the strap of the dynamometer to the rotation point of the knee joint and elbow joint, respectively). The joint torques were normalized for body mass $(\mathrm{Nm} / \mathrm{kg})$. The maximal normalized joint torques out of three trials for knee extension and elbow flexion was used in the analyses.

\section{Covariates}

Questionnaires were conducted to collect information on age (in years), sex, educational level, smoking behavior, alcohol consumption, cardiovascular disease history (CVD), self-reported physical functioning, and health status. Educational level was divided into low, middle, and high. Smoking behavior was divided into three categories: nonsmoker, former smokers, and current smokers. Alcohol consumption was divided into three categories: non-consumers, low-consumers (for women $\leq 7$ glasses alcohol per week; for men $\leq 14$ glasses alcohol per week), and high-consumers (for women $>7$ glasses per week; for men $>14$ glasses alcohol per week). CVD was defined as a (selfreported) history of any of the following conditions: myocardial infarction, cerebrovascular infarction or hemorrhage, percutaneous artery angioplasty of, or vascular surgery on, the coronary, abdominal, peripheral or carotid arteries. Self-reported physical functioning was based on the physical function score, ranging from 0-100, as obtained from the 36-Item Short Form Health Survey (SF-36). Health status was obtained from selfreported general health status on a 5-point scale ranging from 'weak' to 'excellent'. BMI was calculated as: body mass $(\mathrm{kg}) /$ height $(\mathrm{m})^{2}{ }^{2}$. For this, mass and height were measured to the nearest of $0.5 \mathrm{~kg}$ or $0.1 \mathrm{~cm}$ during physical examination. To determine type 2 diabetes, all participants (except those who use insulin) underwent a standardized seven-point oral glucose tolerance test after an overnight fast as described elsewhere. ${ }^{23}$ Type 2 diabetes was defined according to the World Health Organization 2006 criteria. ${ }^{26}$ Participants, without type 1 diabetes, currently using glucose lowering medication were also considered as having type 2 diabetes.

\section{Statistical analyses}

Descriptive statistics were presented for the included population and according to sex. Normally distributed variables were presented as mean (SD), skewed variables were presented as median [25-75\%]. Percentages were provided for categorical variables.

Linear regression analyses were performed to assess the associations of ST, number of sedentary breaks, average sedentary bout duration, and number of prolonged seden- 
tary bouts, total PA and HPA with the physical function measures. Associations were expressed as regression coefficients (B) with 95\% confidence intervals. The associations in models 1 were adjusted for waking time, age, sex, education level, and type 2 diabetes (to account for oversampling in the study design). To assess if the associations were mutually independent, in models 2 HPA, was added to the models describing ST, ST was added in the models describing the associations of HPA (due to collinearity models of total PA were not adjusted for ST), and ST and HPA were both added in the models describing sedentary breaks, mean sedentary bout duration and number of prolonged sedentary bouts. Models 3 were additionally adjusted for several healthrelated factors: BMI, alcohol use, smoking status, CVD history, and health status. We chose to add these health-related factors in models 3 as some of these factors may cause overadjustment bias (in particular BMI and health status). For the ease of interpretation we chose to express the associations of ST, total PA and HPA per one hour. In additional analyses, we have standardized these three exposure variables to allow a better comparison of strengths of the associations. Additionally, the analyses were repeated after excluding all participants with $<4$ valid days of activPAL data $(n=78)$ and after excluding participants who reported functional limitations, defined as having difficulty walking $500 \mathrm{~m}$ or climbing one flight of stairs as reported on the SF-36 ( $\mathrm{n}=328)$. All analyses were performed using IBM SPSS Statistics for Windows, Version 22.0. (Armonk, NY, USA: IBM Corp).

\section{Results}

Of the 1,932 participants, $51.4 \%$ were men. The mean ( \pm SD) age was 59.7(8.2) years and BMI was 26.8(4.4) (Table 1). In over 95\% of participants four or more days with valid accelerometer data were obtained. During waking time 9.4(1.6) hours/day were spent in sedentary positions and 2.0(0.7) hours/day were spent in PA. The remainder of time was spent standing. Women spent less time in sedentary behavior and more time in HPA than men. Mean TCSTtime was similar between men and women. Mean 6MWD and strength measures were greater for men compared with women. When strength measures were adjusted for body mass the differences between sexes were reduced, but relative measures of strength were still greater in men. 
Table 2.1: Descriptive characteristics of the study population $(\mathrm{N}=1,932)$

\begin{tabular}{|c|c|c|c|c|c|c|}
\hline & \multicolumn{2}{|c|}{ Total population } & \multicolumn{2}{|c|}{ Men $(\mathrm{n}=993)$} & \multicolumn{2}{|c|}{ Women $(\mathrm{n}=939)$} \\
\hline Age & 59.7 & $(8.2)$ & 60.8 & $(8.1)$ & 58.6 & $(8.1)$ \\
\hline Educational level (\% high ) & 39.3 & & 43.5 & & 34.9 & \\
\hline Smoking status (\% current) & 12.5 & & 13.5 & & 11.5 & \\
\hline Alcohol consumption (\% high) & 26.3 & & 23.7 & & 29.2 & \\
\hline BMI & 26.8 & (4.4) & 27.5 & $(4.0)$ & 26.1 & (4.6) \\
\hline History of CVD (\%) & 15.7 & & 18.8 & & 12.4 & \\
\hline Type 2 diabetes mellitus (\%) & 26.0 & & 36.0 & & 15.5 & \\
\hline SF-36 physical function score & 95 & {$[85-100]$} & 95 & {$[85-100]$} & 95 & [80-100] \\
\hline Valid days accelerometer data (n) & 6.3 & 1.2 & 6.3 & 1.2 & 6.4 & 1.1 \\
\hline Waking time $(\mathrm{h} /$ day $)$ & 15.7 & $(0.9)$ & 15.8 & $(0.9)$ & 15.7 & $(0.9)$ \\
\hline Sedentary time (h/day) & 9.4 & (1.6) & 9.9 & (1.5) & 8.8 & (1.6) \\
\hline Total PA (h/day) & 2.0 & $(0.7)$ & 2.0 & $(0.7)$ & 2.1 & $(0.6)$ \\
\hline High intensity PA (min/day) & 19.2 & [9.6-32.0] & 14.1 & [6.9-26.5] & 23.6 & [14.5-35.7] \\
\hline Sedentary breaks (N/day) & 37.6 & $(8.5)$ & 37.7 & $(9.0)$ & 37.5 & $(8.0)$ \\
\hline $\begin{array}{l}\text { Average sedentary bout duration } \\
\text { (min) }\end{array}$ & 11.1 & 3.4 & 11.8 & $(3.7)$ & 10.4 & $(2.9)$ \\
\hline Sedentary bouts $\geq 30 \mathrm{~min}(\mathrm{~N} /$ day $)$ & 4.8 & $(1.5)$ & 5.1 & $(1.6)$ & 4.5 & $(1.4)$ \\
\hline $6 \mathrm{MWT}$ distance $(\mathrm{m})$ & 585.1 & $(80.5)$ & 594.1 & $(86.0)$ & 575.5 & (73.0) \\
\hline Timed chair stand test (s) & 23.8 & $(5.5)$ & 23.8 & $(5.7)$ & 23.7 & $(5.2)$ \\
\hline Grip strength $(\mathrm{kg})$ & 35.7 & (10.6) & 43.6 & $(8.1)$ & 27.4 & $(5.4)$ \\
\hline Normalized grip strength $\left(\mathrm{kg} \mathrm{kg}^{-1}\right)$ & 0.45 & $(0.12)$ & 0.50 & $(0.11)$ & 0.39 & $(0.09)$ \\
\hline Elbow flexion strength $(\mathrm{Nm})$ & 59.2 & (23.5) & 73.2 & $(21.4)$ & 44.2 & (14.5) \\
\hline Normalized elbow flexion $\left(\mathrm{Nm} \mathrm{kg}^{-1}\right)$ & 0.75 & $(0.27)$ & 0.86 & $(0.26)$ & 0.64 & $(0.22)$ \\
\hline Knee extension strength (Nm) & 134.9 & $(44.8)$ & 161.5 & $(39.8)$ & 106.9 & $(30.4)$ \\
\hline Normalized knee extension $\left(\mathrm{Nm} \mathrm{kg}^{-1}\right)$ & 1.72 & $(0.48)$ & 1.88 & $(0.46)$ & 1.54 & $(0.45)$ \\
\hline
\end{tabular}

BMI, body mass index; CVD, cardiovascular disease; PA, physical activity; 6MWT, six minute walk test. Values expressed as mean (SD), median [25-75\%], or percentages.

Table 2.2 describes the associations of the sedentary behavior variables (sedentary time, sedentary breaks, average sedentary bout duration and prolonged sedentary bouts) with measures of physical function. An additional hour of ST was associated with shorter 6MWD (B = -2.69 m (95\% CI $=-4.69 ;-0.69)$ and lower elbow flexion strength (B $\left.=-0.01 \mathrm{Nm} \mathrm{kg}^{-1}(-0.02 ; 0.00)\right)$ independent of HPA and other potential confounders (model 3). Every 10 additional sedentary breaks per day were associated with better $\mathrm{TCST}_{\text {time }}(\mathrm{B}=-0.55 \mathrm{~s}(-0.85 ;-0.26))$ in model 3 , but not with the other measures of physical function. A longer average sedentary bout duration was associated with poorer performance on the $\operatorname{TCST}_{\text {time }}(\mathrm{B}=0.17 \mathrm{~s}(0.09 ; 0.25))$ and with lower relative knee extension strength $\left(\mathrm{B}=-0.01 \mathrm{Nm} \mathrm{kg}^{-1}(-0.02 ; 0.00)\right)$. 


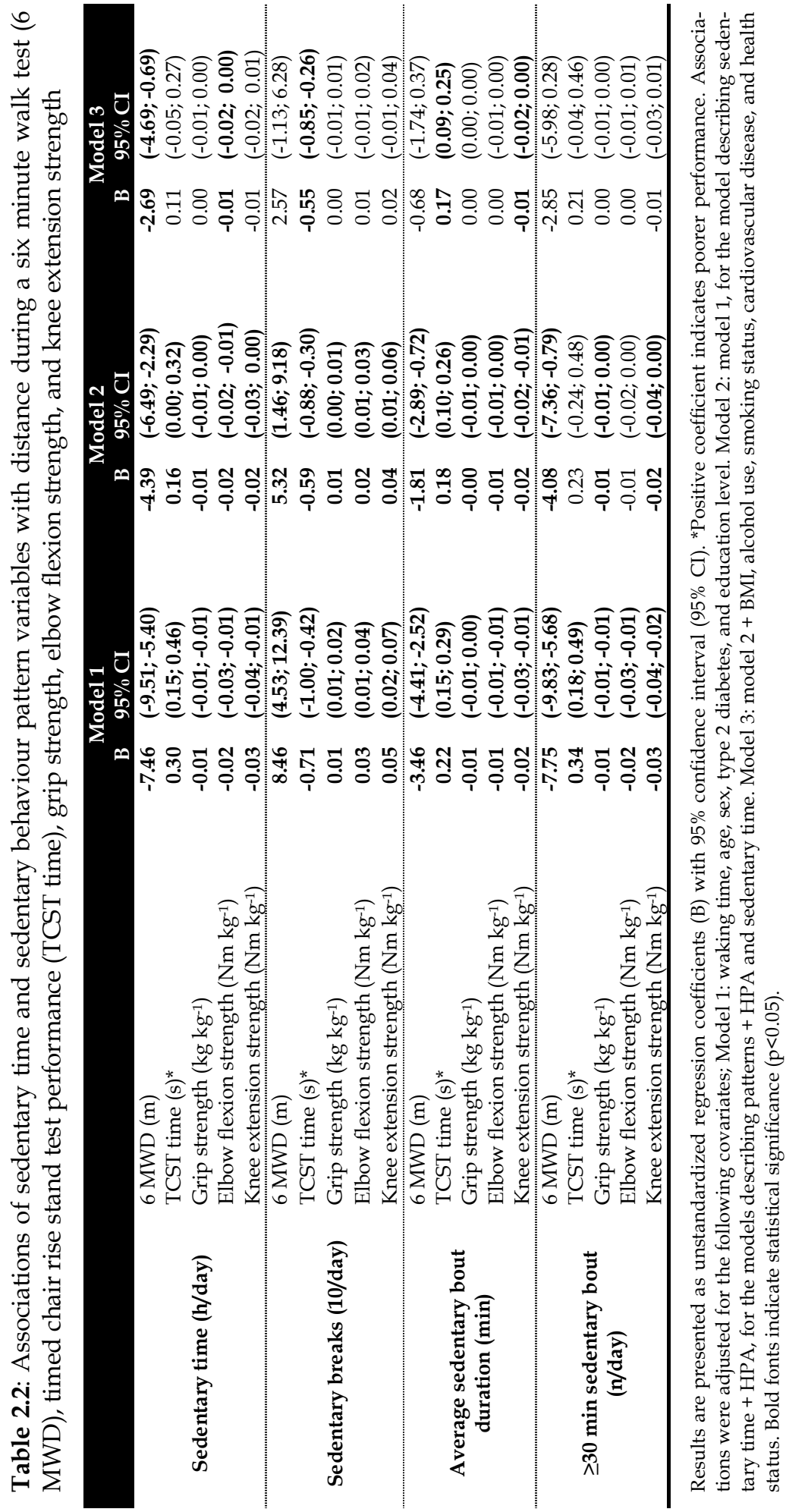


Table 2.3 describes the associations of total PA and HPA with measures of physical function. Total PA was associated with all the different physical function outcome measures in models 1. After additional adjustment for BMI, alcohol use, smoking status, cardiovascular disease, and health status (models 3 ) an additional hour of total PA was statistically significant associated with longer 6MWD (B=16.45 m (11.89; 21.02), better TCSTtime $(B=-0.67 \mathrm{~s}(-1.03 ;-0.30))$, and greater elbow flexion strength $(B=0.03$ $\mathrm{Nm} \mathrm{kg-1}(0.01 ; 0.07))$ and knee extension strength $(B=0.04 \mathrm{Nm} \mathrm{kg-1}(0.01 ; 0.07))$. Associations between HPA and physical function were observed independent of ST in models 2 . In the fully adjusted models (models 3 ) an additional hour of HPA was associated with longer 6MWD $(B=40.72 \mathrm{~m}(30.18 ; 51.25))$, TCSTtime $(\mathrm{B}=-2.25 \mathrm{~s}(-3.09 ;-1.41))$, and greater relative elbow flexion strength $(B=0.05 \mathrm{Nm} \mathrm{kg}-1(0.01 ; 0.08))$ and knee extension strength $\left(B=0.13 \mathrm{Nm} \mathrm{kg}^{-1}(0.06 ; 0.20)\right)$.

To allow a better comparison of the strength of the associations of ST, total PA, and HPA with the physical function outcomes, differences in physical function outcomes were expressed per one standard deviation (SD) of ST, total PA, and HPA. Results are presented in the supplement as table S2.1 and underline that associations of total PA and HPA with physical function were stronger than associations of ST with physical function.

All analyses were repeated after excluding participants with manifest functional limitations $(n=328)$. The association between ST and 6MWD was attenuated and no longer significant $(\mathrm{B}=-2.42(-6.72 ; 1.86))$. Other results were similar as described above (data not tabulated). Additionally, results were similar after excluding participants with $<4$ valid days of accelerometer monitoring $(n=78)$. 


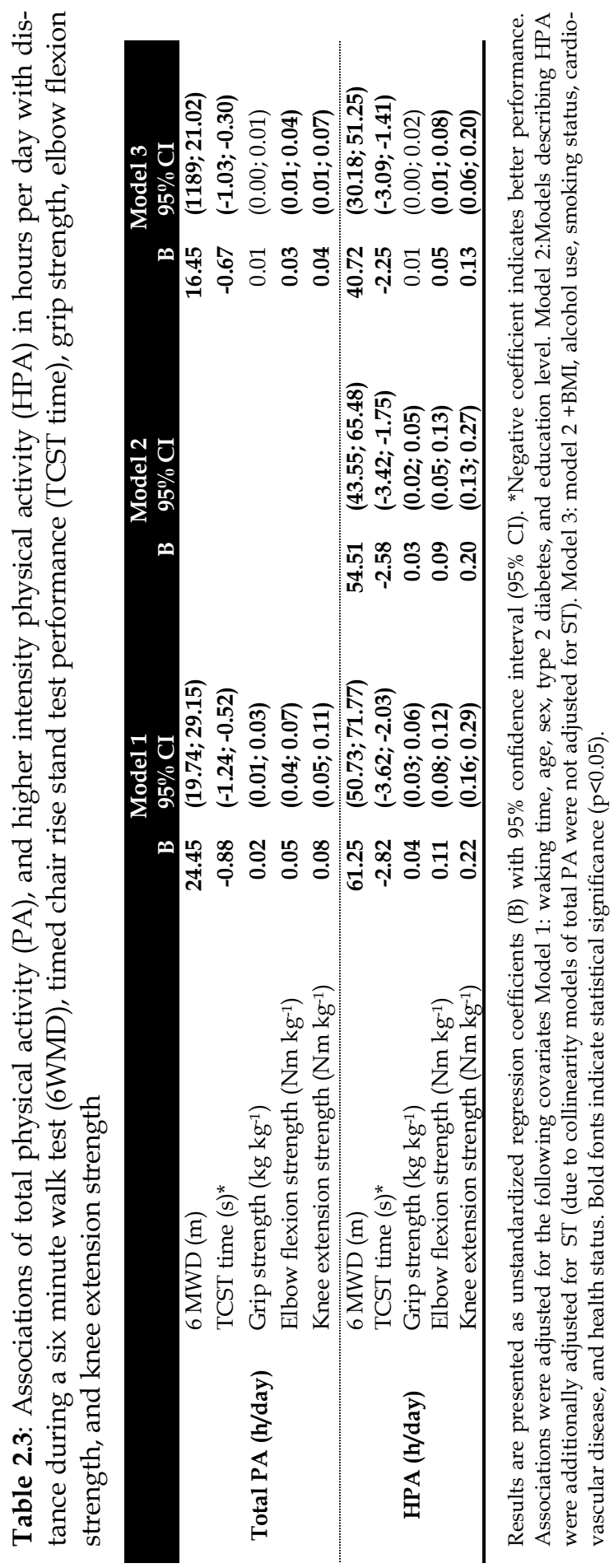




\section{Discussion}

This study examined the associations of objectivity measured (patterns of) sedentary behavior, PA and HPA with physical function in a large sample of adults aged 40-75 years. Our results showed that a larger amount of ST was associated with shorter $6 \mathrm{MWD}$, and lower grip strength and elbow flexion strength. Additionally, more sedentary breaks were associated with faster $\mathrm{TCST}_{\text {time. }}$ Longer average sedentary bout dura-

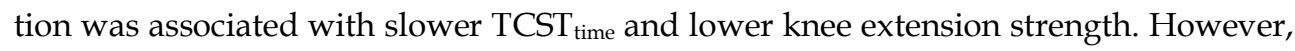
the strength of these associations was relatively weak. PA and HPA were associated

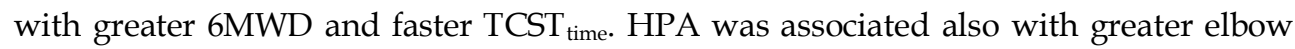
flexion and knee extension strength. The associations of PA and HPA with physical function were stronger than the associations of sedentary behavior variables with physical function.

In our study, we observed a weak association between a large amount of ST and lower physical function. Several other epidemiological studies have examined objectively measured ST as a determinant of physical function expressed as gait speed or chair rise test. ${ }^{13-18,20}$ Findings from these studies were inconsistent as in some studies an association was observed between larger amounts of ST and worse physical function, 13-16,20 whereas in other studies no association was observed. 17,18 To our knowledge, three studies examined associations between objectively measured ST and knee extension strength. ${ }^{8,27,28}$ In agreement with our results, these studies reported no association between ST and knee extension strength. Two other studies reported on associations between objectively measured ST and hand grip strength with different results. Cooper $e t$ al. ${ }^{13}$ did observe an association between ST and grip strength, while Keevil et al..$^{17}$ did not. A difference between our study and the others was that we normalized measures of strength for body mass. Normalization for body mass allows better comparisons of strength measures between individuals of different body sizes. ${ }^{29}$ We argued that an individual with greater body mass needs more strength to carry his/her own weight, thus a relative measure of strength would better reflect physical function. In addition, compared with absolute measures, normalized measures of hand grip strength and knee extension strength have been associated more strongly with functional limitations. 30,31

In this study we observed some associations between the patterns of sedentary behavior with physical function. However, strength of these associations was rather weak. Few other studies have examined patterns of sedentary behavior and associations with physical function. In a small study ( $\mathrm{n}=44$, mean age $70 \pm 8$ years), Genusso et al. reported that the number of sedentary breaks were positively and prolonged sedentary bouts were negatively associated physical function. ${ }^{32}$ In addition, Sardinha et al. reported a 
positive association between sedentary breaks and physical function in a study with older adults (mean age $73 \pm 6$ years). ${ }^{19}$ In contrast, in the study by Reid et al. (mean age $58 \pm 10$ years) sedentary breaks and prolonged sedentary bouts were not associated with physical function. ${ }^{18}$ In our study, which was comparable to the study by Reid et al. in terms of age, we did however observe a small, beneficial association between the number of sedentary breaks and TCST time. $_{\text {. }}$

Inconsistencies in outcomes between studies may have resulted from a difference in study populations. For example, the study by Reid et al., ${ }^{18}$ who reported no association between sedentary behavior and physical function, had the youngest study population (mean age $58 \pm 10$ years). The majority of the studies in which a negative association between large amounts of ST and physical function was reported comprised an older population, with mean age $>65$ years. ${ }^{14-16,20}$ A younger population would generally be healthier and have a higher physical functioning. In our study this was seen by a very high median[25\%-75\%] SF-36 physical function score: 95[85-100]. Consequently the measures of physical function may have a limited range due to a ceiling effect.

Positive associations between PA, in particular HPA, and physical function are in line with the literature as summarized in reviews reviews. ${ }^{4,7}$ Both reviews incorporated longitudinal and/or intervention studies based on self-reported measures of PA. In addition, more recent studies that cross-sectionally examined associations between objectively measured PA and/or MVPA reported a positive association with physical function as well. ${ }^{16,18}$ As mentioned, the strength of the associations of sedentary behavior was small compared with the associations of PA and HPA. It is unlikely that the associations of sedentary behavior represent clinically meaningful differences. For example, in a population of COPD patients, $\sim 30$ meters was found to be the minimal clinically important difference in 6WMD. ${ }^{33}$ In our study each additional hour of sedentary time was associated with 2.69 meters shorter 6MWD.

Future studies in populations of different ages should examine the associations between objectively measured sedentary behavior and physical function. Preferably these studies should have a longitudinal design to establish temporality. Importantly, future studies should provide answer to the important question: how much ST is too much? For instance in bed-rest studies, regarded as extreme conditions of ST, substantial muscle mass loss has been observed. ${ }^{34}$ In our study (and others), ${ }^{18,328,30}$ prolonged bouts of 30 minutes were used, but perhaps 30 minutes is not long enough to negatively affect physical function.

A strength of this study was the use of a posture based accelerometer. The activPAL3 has been found to measure ST and posture transitions (sedentary breaks) more accurately than accelerometers that determine ST based on acceleration data, which have 
been used in the majority of the studies. 35,36 Therefore, estimations of ST were probably more accurate than those in studies using other types of accelerometers. Further, we used multiple objective measures of physical function that reflect upper and lower body function including several measures for muscle strength, but this study is not without limitations. Importantly, due to the cross-sectional study design, caution is required with regard to causal inferences. It cannot be excluded that due to physical limitations, people engage less time in (H)PA and/or more in sedentary behaviors. However, in additional analyses we have demonstrated that after excluding individuals with mobility limitations the majority of the associations persisted. In addition, step frequency was used to determine HPA. This method may be less precise to determine intensity of PA compared with estimations based on acceleration data. However, we used a step frequency of $>110$ steps/minute which has been reported to correspond to a MET score > 3.0 (a commonly used as cut-off value for MVPA). Further, although the actviPAL3 may capture movement and intensity (based on step frequency), it does not provide context of activities. For example, the activPAL3 will classify (strength) training exercises as sedentary when performed in a sitting or lying position. Finally, our study population consisted of a highly functioning population aged 40-75 years of predominantly Caucasians from European descent. This was partly a result from the exclusion of participants that were unable to perform any of the physical function tests. Therefore, generalizability of our results to other populations and ages may be limited. It is not unlikely that associations of sedentary behavior and PA with physical function are different in, for example, frail or institutionalized populations, which have other activity patterns and lower physical function.

In conclusion, in adults aged 40-75 years, sedentary behavior appeared to be marginally associated with lower physical function, independent of HPA. This suggests that merely reducing sedentary behavior is insufficient to improve/maintain physical function. On the other hand, engaging regularly in PA, and in particular HPA, is important for physical function. 


\section{References}

1. Cooper R, Kuh D, Cooper C, Gale CR, Lawlor DA, Matthews F, Hardy R, Falcon, Teams HAS. Objective measures of physical capability and subsequent health: a systematic review. Age Ageing 2011;40(1):14-23.

2. Cooper R, Kuh D, Hardy R, Mortality-Review-Group. Objectively measured physical capability levels and mortality: systematic review and meta-analysis. BMJ 2010;341:c4467.

3. Statistical-Office-of-the-European-Communities. Functional and activity limitations statistics 2015 Accessed 09 Jan, 2017.

4. Paterson DH, Warburton DE. Physical activity and functional limitations in older adults: a systematic review related to Canada's Physical Activity Guidelines. Int. J. Behav. Nutr. Phys. Act. 2010;7(38):38.

5. Warburton DE, Charlesworth S, Ivey A, Nettlefold L, Bredin SS. A systematic review of the evidence for Canada's Physical Activity Guidelines for Adults. Int. J. Behav. Nutr. Phys. Act. 2010;7(1):1.

6. Bauman A, Merom D, Bull FC, Buchner DM, Singh MAF. Updating the evidence for physical activity: summative reviews of the epidemiological evidence, prevalence, and interventions to promote "Active Aging". The Gerontologist 2016;56(Suppl 2):S268-S280.

7. Volkers KM, de Kieviet JF, Wittingen HP, Scherder EJA. Lower limb muscle strength (LLMS): Why sedentary life should never start? A review. Arch. Gerontol. Geriatr. 2012;54(3):399-414.

8. Kahlmeier S, Wijnhoven TMA, Alpiger P, Schweizer C, Breda J, Martin BW. National physical activity recommendations: systematic overview and analysis of the situation in European countries. BMC Public Health 2015;15(1):1-14.

9. Owen N, Healy GN, Matthews CE, Dunstan DW. Too much sitting: the population health science of sedentary behavior. Exerc. Sport Sci. Rev. 2010;38(3):105-13.

10. Sedentary Behaviour Research Network. (2102). Letter to the editor: standardized use of the terms "sedentary" and "sedentary behaviours". Appl. Physiol. Nutr. Metab;37:540-542.

11. Wilmot EG, Edwardson CL, Achana FA, Davies MJ, Gorely T, Gray LJ, Khunti K, Yates T, Biddle SJ. Sedentary time in adults and the association with diabetes, cardiovascular disease and death: systematic review and meta-analysis. Diabetologia 2012;55(11):2895-2905.

12. Brocklebank LA, Falconer CL, Page AS, Perry R, Cooper AR. Accelerometer-measured sedentary time and cardiometabolic biomarkers: A systematic review. Prev. Med. 2015;76:92-102.

13. Cooper AJ, Simmons RK, Kuh D, Brage S, Cooper R, scientific N, data collection t. Physical activity, sedentary time and physical capability in early old age: British birth cohort study. PLoS ONE 2015;10(5):e0126465.

14. Lee J, Chang RW, Ehrlich-Jones L, Kwoh CK, Nevitt M, Semanik PA, Sharma L, Sohn MW, Song J, Dunlop DD. Sedentary behavior and physical function: objective evidence from the Osteoarthritis Initiative. Arthritis Care Res (Hoboken) 2015;67(3):366-73.

15. Rosenberg DE, Bellettiere J, Gardiner PA, Villarreal VN, Crist K, Kerr J. Independent Associations Between Sedentary Behaviors and Mental, Cognitive, Physical, and Functional Health Among Older Adults in Retirement Communities. J. Gerontol. A. Biol. Sci. Med. Sci. 2016;71(1):78-83.

16. Santos DA, Silva AM, Baptista F, Santos R, Vale S, Mota J, Sardinha LB. Sedentary behavior and physical activity are independently related to functional fitness in older adults. Exp. Gerontol. 2012;47(12):908-12.

17. Keevil VL, Cooper AJ, Wijndaele K, Luben R, Wareham NJ, Brage S, Khaw KT. Objective Sedentary Time, Moderate-to-Vigorous Physical Activity, and Physical Capability in a British Cohort. Med. Sci. Sports Exerc. 2016;48(3):421-9.

18. Reid N, Daly RM, Winkler EA, Gardiner PA, Eakin EG, Owen N, Dunstan DW, Healy GN. Associations of Monitor-Assessed Activity with Performance-Based Physical Function. PLoS ONE 2016;11(4):e0153398.

19. Sardinha LB, Santos DA, Silva AM, Baptista F, Owen N. Breaking-up Sedentary Time Is Associated With Physical Function in Older Adults. Journals of Gerontology Series a-Biological Sciences and Medical Sciences 2015;70(1):119-124. 
20. Davis MG, Fox KR, Stathi A, Trayers T, Thompson JL, Cooper AR. Objectively measured sedentary time and its association with physical function in older adults. J. Aging Phys. Act. 2014;22(4):474-81.

21. Matthews CE, Chen KY, Freedson PS, Buchowski MS, Beech BM, Pate RR, Troiano RP. Amount of time spent in sedentary behaviors in the United States, 2003-2004. Am. J. Epidemiol. 2008;167(7):875881.

22. Evenson KR. Objective measurement of physical activity and sedentary behavior among US adults aged 60 years or older. Prev. Chronic. Dis. 2012;9.

23. Schram MT, Sep SJ, van der Kallen CJ, Dagnelie PC, Koster A, Schaper N, Henry RM, Stehouwer $\mathrm{CD}$. The Maastricht Study: an extensive phenotyping study on determinants of type 2 diabetes, its complications and its comorbidities. Eur. J. Epidemiol. 2014;29(6):439-51.

24. van der Berg JD, Willems PJ, van der Velde JHPM, Savelberg HHCM, Schaper NC, Schram MT, Sep SJ, Dagnelie PC, Bosma H, Stehouwer CDA. Identifying waking time in 24-h accelerometry data in adults using an automated algorithm. J. Sports Sci. 2016;34(19):1867-73.

25. Tudor-Locke C, Craig CL, Brown WJ, Clemes SA, De Cocker K, Giles-Corti B, Hatano Y, Inoue S, Matsudo SM, Mutrie N, Oppert JM, Rowe DA, Schmidt MD, Schofield GM, Spence JC, Teixeira PJ, Tully MA, Blair SN. How many steps/day are enough? For adults. Int. J. Behav. Nutr. Phys. Act. 2011;8(1):79.

26. World Health Organization. Definition and Diagnosis of Diabetes Mellitus and Intermediate Hyperglycemia. 2006.

27. Willoughby $\mathrm{T}$, Copeland JL. Sedentary time is not independently related to postural stability or leg strength in women 50-67 years old. Applied Physiology Nutrition and Metabolism 2015;40(11):11231128.

28. Foong YC, Chherawala N, Aitken D, Scott D, Winzenberg T, Jones G. Accelerometer-determined physical activity, muscle mass, and leg strength in community-dwelling older adults. J Cachexia Sarcopenia Muscle 2016;7(3):275-83.

29. Jaric S. Muscle strength testing: use of normalisation for body size. Sports Med. 2002;32(10):615-31.

30. Dong R, Wang X, Guo Q, Wang J, Zhang W, Shen S, Han P, Ma Y, Kang L, Wang M, Fu L, Jia L, Wang L, Niu K. Clinical Relevance of Different Handgrip Strength Indexes and Mobility Limitation in the Elderly Adults. J. Gerontol. A. Biol. Sci. Med. Sci. 2016;71(1):96-102.

31. Barbat-Artigas S, Rolland Y, Cesari M, Abellan van Kan G, Vellas B, Aubertin-Leheudre M. Clinical relevance of different muscle strength indexes and functional impairment in women aged 75 years and older. J. Gerontol. A. Biol. Sci. Med. Sci. 2013;68(7):811-9.

32. Gennuso KP, Thraen-Borowski KM, Gangnon RE, Colbert LH. Patterns of sedentary behavior and physical function in older adults. Aging Clin. Exp. Res. 2016;28(5):943-50.

33. Polkey MI, Spruit MA, Edwards LD, Watkins ML, Pinto-Plata V, Vestbo J, Calverley PM, TalSinger R, Agusti A, Bakke PS, Coxson HO, Lomas DA, MacNee W, Rennard S, Silverman EK, Miller BE, Crim C, Yates J, Wouters EF, Celli B, Evaluation of CLtIPSESI. Six-minute-walk test in chronic obstructive pulmonary disease: minimal clinically important difference for death or hospitalization. Am. J. Respir. Crit. Care. Med. 2013;187(4):382-6.

34. Dirks ML, Wall BT, van de Valk B, Holloway TM, Holloway GP, Chabowski A, Goossens GH, van Loon LJ. One week of bed rest leads to substantial muscle atrophy and induces whole-body insulin resistance in the absence of skeletal muscle lipid accumulation. Diabetes 2016:db151661.

35. Berendsen BAJ, Hendriks MRC, Meijer K, Plasqui G, Schaper NC, Savelberg HHCM. Which activity monitor to use? Validity, reproducibility and user friendliness of three activity monitors. BMC Public Health 2014;14(749).

36. Kozey-Keadle S, Libertine A, Lyden K, Staudenmayer J, Freedson PS. Validation of wearable monitors for assessing sedentary behavior. Med. Sci. Sports Exerc. 2011;43(8):1561-7. 
Supplement to chapter 2 


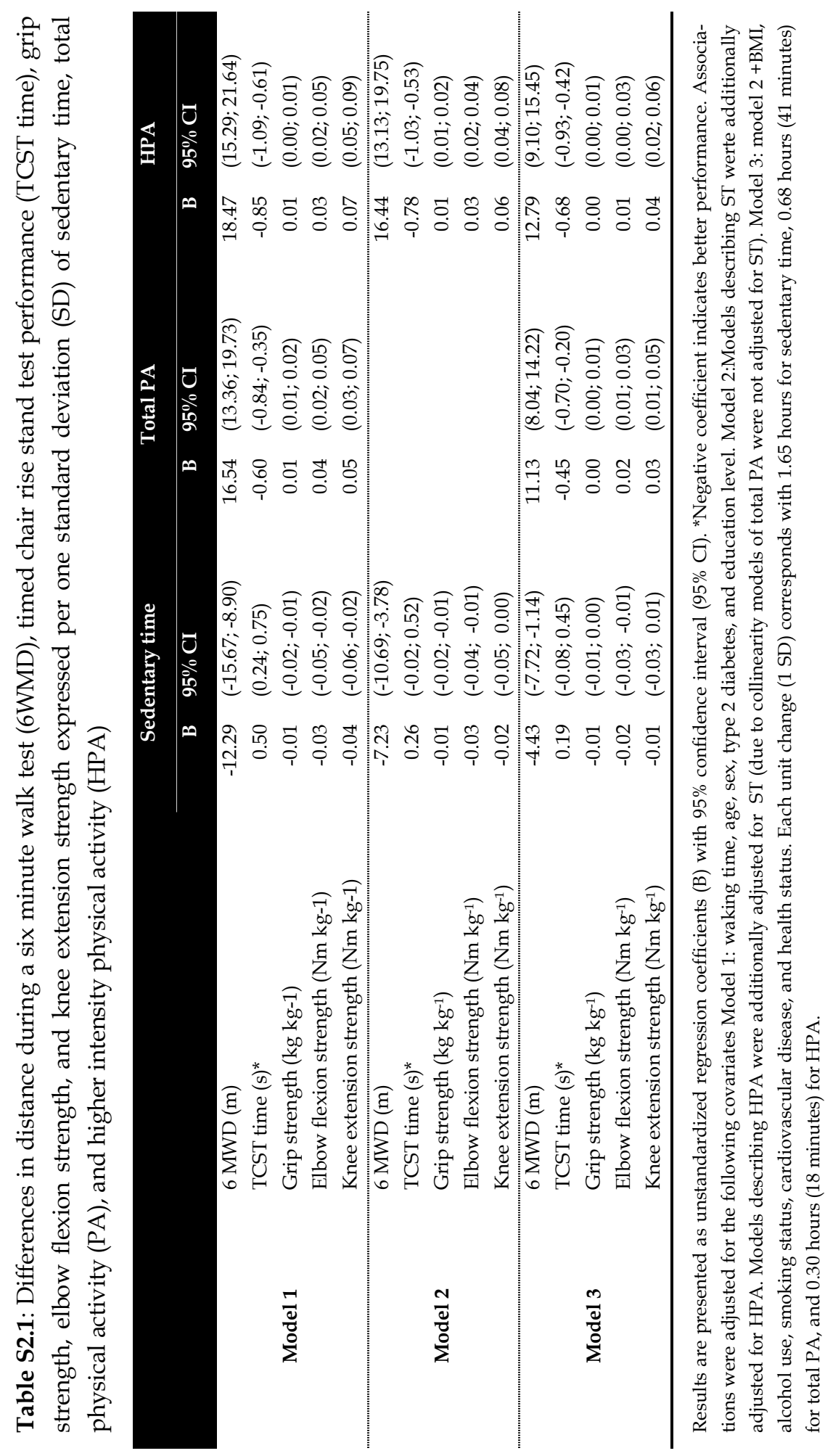





\section{Chapter 3}

\section{Sedentary behavior, physical activity and fitness - The Maastricht Study -}

Jeroen HPM van der Velde, Annemarie Koster, Julianne D van der Berg, Simone JS Sep, Carla JH van der Kallen, Pieter C Dagnelie, Miranda T Schram, Ronald MA Henry, Simone JPM Eussen Martien CJM van Dongen, Coen DA Stehouwer, Nicolaas C Schaper, Hans HCM Savelberg 


\section{Abstract}

Purpose: This cross-sectional study examined the mutual independent associations of sedentary behaviour, lower intensity physical activity (LPA) and higher intensity physical activity HPA (an approximation of moderate to vigorous physical activity (MVPA) with cardio-respiratory fitness (CRF).

Methods: 2,024 participants were included from The Maastricht Study (mean \pm SD age: $59.7 \pm 8.1$ years, $49.6 \% \mathrm{men}$ ). With the activPAL3 activity monitor we assessed sedentary time (ST), sedentary pattern variables (number of sedentary breaks, average sedentary bout duration, and number of prolonged sedentary bouts ( $\geq 30 \mathrm{~min})$ ), LPA, and HPA. CRF was calculated as maximum power output per $\mathrm{kg}$ body mass $\left(\mathrm{W}_{\max } \mathrm{kg}^{-1}\right)$ estimated from a sub-maximal cycle ergometer test. Linear regression analyses and isotemporal substitution analyses were used to examine associations of ST, sedentary pattern variables, and HPA with CRF. Analyses were stratified by sex.

Results: One hour of ST per day was associated with a lower $W_{\max } \mathrm{kg}^{-1}: B_{\text {men }}=-0.03$ $(95 \%$ CI: $-0.05 ;-0.01)$ and $B_{\text {women }}=-0.02(-0.04 ; 0.00)$, independent of HPA. No statistically significant associations between sedentary patterns variables and CRF were observed. LPA was associated with a higher $\mathrm{W}_{\max } \mathrm{kg}^{-1}: \mathrm{B}_{\operatorname{men}}=0.12(0.07 ; 0.17)$ and $\mathrm{B}_{\text {women }}=$ $0.12(0.07 ; 0.18)$. HPA was associated with a higher $W_{\max } \mathrm{kg}^{-1}: B_{\operatorname{men}}=0.48(0.38 ; 0.58)$ and $\mathrm{B}_{\text {women }}=0.27(0.18 ; 0.36)$. Replacing ST with LPA ( $\mathrm{B}_{\text {men }}=0.08(0.03 ; 0.14), \mathrm{B}_{\text {women }}=0.10$ $(0.05 ; 0.16))$ or with HPA $\left(B_{\text {men }}=0.49(0.39 ; 0.59), B_{\text {women }}=0.28(0.19 ; 0.36)\right)$, but not with standing was associated with higher CRF.

Conclusion: Modest associations between sedentary behaviour and CRF were observed. Replacing ST with LPA was associated with higher CRF, which could be of particular importance for individuals who cannot engage in HPA. Nonetheless, replacing ST with HPA was associated with greatest estimated change in CRF. 


\section{Introduction}

Cardio-respiratory fitness (CRF), defined as the capacity of the cardiovascular and respiratory systems to supply fuel and oxygen during sustained physical activity (PA), has shown to be an important determinant of health. Impaired CRF has been recognized in the aetiology of the metabolic syndrome and cardiovascular disease, and may predict premature mortality. ${ }^{1-4}$ Although CRF is determined to a certain extent by factors such as sex, genetics and environment, physical activity (PA) has been identified as a key modifiable determinant. ${ }^{5}$ Specifically, more time engaged in moderate to vigorous physical activity (MVPA), has been associated with higher CRF.6,7

Despite the well-documented health benefits of MVPA, PA guidelines are frequently not met and the majority of the adult population appears to be sedentary most time of the day. ${ }^{8,9}$ In addition, even individuals who do regularly engage in MVPA may spend the majority of the day in sedentary behaviour. Sedentary behaviour is defined as any waking behaviour that is characterized by an energy expenditure $\leq 1.5$ METs (metabolic equivalent of task) while in a sitting or reclining position. ${ }^{10}$ More daily sedentary time (ST) has been associated with several adverse health outcomes including an increased risk for the metabolic syndrome, type 2 diabetes and cardiovascular disease.11,12 Importantly, these associations were observed independent of MVPA. Even individuals who do adhere to PA guidelines appear to be at increased risk for detrimental health effects from sitting too much. ${ }^{9}$ These findings are supported by the observation that MVPA may not compensate the detrimental associations of ST on markers of metabolic health. ${ }^{13}$

Not only total ST has shown to be a determinant of several adverse health outcomes, the pattern in which ST is accumulated may be relevant as well. Patterns of ST can be expressed by sedentary breaks (interruptions of periods of sitting), the length of sedentary bouts (uninterrupted periods of sedentary time) and the mean duration of a sedentary bout. For instance, more sedentary breaks have been associated with favourable cardio-metabolic outcomes. ${ }^{14,15}$ Since both sedentary behaviour and CRF have been associated with detrimental health, associations between these constructs should be examined. Possibly, the positive effect of MVPA on CRF may be attenuated by increased amounts of ST. To date, only a few studies have considered the associations of sedentary behaviour with CRF. Studies using self-reported measures of ST, observed that larger amounts of ST were associated with lower CRF.16,17 One study that used objectively measured ST and CRF in a population aged 12-49 years, also found that more ST was associated with a lower CRF. ${ }^{18}$ 
To obtain a better insight into the associations between sedentary behaviour and CRF, we examined the mutually independent associations of objectively measured ST, sedentary behaviour patterns and PA with CRF in a large adult population. PA was divided into lower intensity (LPA) and higher intensity physical activity (HPA) (corresponding approximately with MVPA). We hypothesized that more daily ST, a longer average sedentary bout duration and more prolonged sedentary bouts per day would be associated with lower CRF. On the other hand, more daily sedentary breaks and more time engaged in LPA and HPA would be associated with higher CRF.

\section{Methods}

\section{Population}

We used data from The Maastricht Study, an observational prospective populationbased cohort study. The rationale and methodology have been described previously. ${ }^{19}$ In brief, the study focuses on the aetiology, pathophysiology, complications and comorbidities of type 2 diabetes mellitus (T2DM) and is characterized by an extensive phenotyping approach. Eligible for participation were all individuals aged between 40 and 75 years and living in the southern part of the Netherlands. Participants were recruited through mass media campaigns and from the municipal registries and the regional Diabetes Patient Registry via mailings. Recruitment was stratified according to known T2DM status, with an oversampling of individuals with T2DM, for reasons of efficiency. The present report includes cross-sectional data from a selection of the first 3451 participants, who completed the baseline survey between November 2010 and September 2013. For this study data were available for 2,024 participants. Main reasons for missing data were: medical exclusion for the sub-maximal cycle ergometer test $(n=425)$, invalid ergometer test $(n=180)$, missing or invalid accelerometry data $(n=629)$ and missing data in covariates $(n=193)$. The examinations of each participant were performed within a time window of three months. The study has been approved by the institutional medical ethical committee (NL31329.068.10) and the Minister of Health, Welfare and Sports of the Netherlands (Permit 131088-105234-PG). All participants gave written informed consent.

\section{Accelerometry: ST, patterns of sedentary behaviour, and HPA}

Daily activity levels were measured using the activPAL3 ${ }^{\mathrm{TM}}$ physical activity monitor (PAL Technologies, Glasgow, UK). The activPAL3 is a small $(53 \times 35 \times 7 \mathrm{~mm})$, lightweight (15 g) triaxial accelerometer that records movement in the vertical, anteroposterior and mediolateral axes, and also determines posture (sitting or lying, standing and stepping) based on acceleration information. The device was attached directly to the skin on the front of the right thigh with transparent $3 \mathrm{M}$ Tegaderm ${ }^{\mathrm{TM}}$ tape, after the device had been waterproofed using a nitrile sleeve. Participants were instructed to 
wear the accelerometer for 8 consecutive days, without removing it at any time. To avoid inaccurately identifying non-wear time, participants were asked not to replace the device once removed. Data were uploaded using the activPAL software and processed using customized software written in MATLAB R2013b (MathWorks, Natick, MA, USA). Data from the first day were excluded from the analysis because participants performed physical function tests at the research centre after the device was attached. In addition, data from the final wear day providing $\leq 14$ waking hours of data were excluded from the analysis. Participants were included if they provided at least 1 valid day ( $\geq 10 \mathrm{~h}$ of waking data).

The total amount of ST was based on the sedentary posture (sitting or lying), and calculated as the mean time spent in a sedentary position during waking time per day. The method used to determine waking time has been described elsewhere. ${ }^{20}$ The number of sedentary breaks during waking time was determined as each transition from a sitting or lying position to standing or stepping with a duration of at least $1 \mathrm{~min}$, and the mean number of breaks per day was calculated. Sedentary time accumulated in a consecutive period $\geq 30 \mathrm{~min}$ was defined as a prolonged sedentary bout, and the mean number of prolonged sedentary bouts during waking time per day was calculated. Average bout duration was calculated by dividing total sedentary time by total number of sedentary bouts of any duration. The total amount of standing time was based on the standing posture, and calculated as the mean time spent standing during waking time per day. The total amount of stepping (physical activity) was based on the stepping posture, and calculated as the mean time stepping during waking time per day. Stepping time (physical activity) was further classified into higher intensity physical activity (HPA; minutes with a step frequency $>110$ steps/min during waking time) and lower intensity physical activity (LPA; minutes with a step frequency $\leq 110$ steps/min during waking time). ${ }^{21}$

\section{Sub-maximal cycle ergometer test: CRF}

As an objective measure of CRF estimated maximum power output adjusted for body mass $\left(\mathrm{W}_{\max } \mathrm{kg}^{-1}\right)$ was used. ${ }^{22,23} \mathrm{~W}_{\max }$ was estimated from a graded sub-maximal exercise protocol performed on a cycle ergometer system (CASETM version 6.6 in combination with e-bike, GE Healthcare, Milwaukee, WI, USA). Participants were excluded from the sub-maximal cycle ergometer test if they had experienced cardiovascular complications in the preceding 3 months, had an abnormal resting ECG, were known with cardiovascular complications such as pericarditis and hypertrophic cardiomyopathy, had severe hypertension (SBP $\geq 180$ and/or DBP $\geq 110$ ), renal failure or an ICD/pacemaker. Participants eligible for the test were fitted with a blood pressure cuff on the upper left arm (Suntech Tango+TM, SunTech Medical, Inc. Morisville, NC, USA) and electrodes on the thorax to provide continuously a 12-leads ECG. 
The protocol consisted of a short warm-up period and at most 7 stages with increasing work load. Participants were instructed to cycle at a cadence of 60-70 rotation per minute (rpm) during a short familiarization period without any external workload. For the first exercise stage, external workload was set at $25 \mathrm{~W}$. Every consecutive 2 minutes external workload was increased with $25 \mathrm{~W}$. At the end of each stage, heart rate (HR) and blood pressure were measured. Further, the participant was asked to provide a rating of perceived exertion (RPE) on the 15-point Borg-scale; an interval scale ranging from 6 ('no exertion at all') up to 20 ('maximal exertion'). The exercise protocol was considered as 'completed' when HR reached $\geq 85 \%$ of the estimated maximum HR $\left(\mathrm{HR}_{\max }, 220\right.$-age) or when a $\mathrm{RPE} \geq 17$ was scored by the participant. If $\mathrm{HR}<85 \%$ or $\mathrm{RPE}$ $<17$ by the end of stage 7 (work load of $175 \mathrm{~W}$ ), the test was also stopped. The test could also be prematurely terminated on medical grounds or when the participant was unwilling to continue.

Submaximal values of HR and RPE with workload from each stage were extrapolated to $100 \%$ of maximum HR or an RPE of 20 and corresponding workload ( $\left.=W_{\max }\right)$ using individual linear regression models. Using RPE to predict $W_{\max }$ overcomes the issue that certain medical conditions, such as autonomous neuropathy and medication use (e.g. beta blockers) may affect the linear association of HR with power output. Consequently, this protocol is suitable for participants who otherwise would have been excluded from exercise testing. ${ }^{24}$ Analyses demonstrated that estimated $W_{\max }$ using $\mathrm{HR}$ $\left(\mathrm{W}_{\max } \mathrm{HR} 85 \%\right.$ ) was comparable to $\mathrm{W}_{\max }$ based on RPE ( $\mathrm{W}_{\max }$ RPE17) in this study (see text and figure S3.1 in supplement S3.1).

$W_{\max }$ was calculated from $H R$ values if the test was completed based on HR, i.e. HR $\geq 85 \%$ of estimated $\mathrm{HR}_{\max }\left(\mathrm{W}_{\max } \mathrm{HR} 85 \% ; \mathrm{N}=1,201\right)$. $\mathrm{W}_{\max }$ was calculated from RPE values if the test was completed based on RPE, i.e. RPE $\geq 17$ ( $\left.\mathrm{W}_{\max } \mathrm{RPE} 17 ; \mathrm{N}=350\right)$. In addition to completed tests, $W_{\max }$ from uncompleted tests was calculated from HR if $\geq 75 \%$ of $\mathrm{HR}_{\max }$ was achieved $\left(\mathrm{W}_{\max } \mathrm{HR} 75 \%\right.$; $\left.\mathrm{N}=375\right)$ and $\mathrm{W}_{\max }$ was calculated from RPE values if an $R P E \geq 15$ was scored ( $\left.W_{\max } R P E 15 ; N=98\right)$. Estimations of $W_{\max }$ from these lower ranges of HR and RPE were found to be similar to completed tests (methods and results of the analyses between $W_{\max } \mathrm{HR} 85 \%$ versus $W_{\max } H R 75 \%$ and $W_{\max } R P E 17$ versus $W_{\text {max }} R P E 15$ are shown as text and table in supplement S3.2). Tests where both $75 \%$ of $\mathrm{HR}_{\max }$ and RPE15 were not achieved were considered as invalid.

\section{Covariates}

The following variables were considered as potential confounders: BMI, age, education level, alcohol use, smoking status, cardiovascular disease, energy intake, mobility limitations, beta-blocker use, and type 2 diabetes. BMI was calculated from weight and height measured in a physical examination to the nearest of $0.5 \mathrm{~kg}$ or $0.1 \mathrm{~cm}$. Question- 
naires were conducted to collect information on age (in years), sex, educational level, smoking status, alcohol consumption, cardiovascular disease (CVD) history, energy intake and mobility limitations. Educational level was divided into low, middle, and high. Smoking status was divided into current, former, and never smokers. Alcohol consumption was divided into three categories: non-consumers, low-consumers (for women $\leq 7$ glasses alcohol per week; for men $\leq 14$ glasses alcohol per week), and highconsumers (for women $>7$ glasses per week; for men $>14$ glasses alcohol per week). ${ }^{25}$ CVD history was derived from the Rose questionnaire and defined as a self-reported history of any of the following conditions: myocardial infarction, cerebrovascular infarction or haemorrhage, percutaneous artery angioplasty of, or vascular surgery on, the coronary, abdominal, peripheral or carotid arteries. Energy intake was derived from a food frequency questionnaire, which was developed for The Maastricht Study, and calculated as the mean energy intake per day (kcal). Mobility limitation was acquired from the Dutch version of the Short Form Health Survey (SF-36) and was defined as having difficulty with stair climbing and/or walking $500 \mathrm{~m} .{ }^{26}$ The use of diabetes medication and beta blockers was obtained from a medication interview. To determine glucose metabolism status, all participants (except those who use insulin or with a fasting plasma glucose $>11.0 \mathrm{mmol} / \mathrm{L}$ ) underwent a standardized seven-point oral glucose tolerance test after an overnight fast as described elsewhere ${ }^{19}$. Glucose metabolism was defined according to the World Health Organization 2006 criteria, and participants were categorized as having a normal glucose metabolism, prediabetes, type 2 diabetes, or type 1 diabetes mellitus (and other types). Participants on diabetes medication and without type 1 diabetes were also considered as having type 2 diabetes. ${ }^{27}$

\section{Statistical analyses}

CRF $\left(\mathrm{W}_{\max } \mathrm{kg}^{-1}\right.$ ) was used as a continuous measure and was categorized into tertiles (low, medium, and high fit) based on sex and age; (40-49, 50-59, 60-69, and >70 years). Descriptive statistics are presented for the total population and by sex. Normally distributed variables are presented as mean (SD), skewed variables are presented as median [Interquartile range (IQR) 25-75\%]. Percentages are provided for categorical variables.

Linear regression analyses were performed to assess the association between ST, sedentary breaks, average sedentary bout duration, number of prolonged sedentary bouts, LPA and HPA with estimated $W_{\max } \mathrm{kg}^{-1}$. Associations were expressed as regression coefficients (B) with 95\% confidence intervals. The associations in models 1 were adjusted for waking time, age, education level, and type 2 diabetes (to account for oversampling in the study design). In models 2 HPA was added to the model describing ST, and vice versa. ST and HPA were both added in the models describing sedentary breaks, average sedentary bout duration and number of prolonged sedentary bouts. In 
models describing LPA no other activity was added due to collinearity. Models 3 were additionally adjusted for BMI, alcohol use, smoking status, cardio vascular disease, beta-blocker use, energy intake and mobility limitations. Interaction terms with sex were significant for ST, prolonged sedentary bouts and HPA $(p<0.10)$. Analyses were therefore stratified by sex. Additional analyses were performed with participants that provided $\geq 4$ valid days of activPAL data $(n=1,940)$ and with participants that had complete ergometer tests $(n=1,551)$.

An isotemporal substitution modelling approach was applied to explore the effect of theoretical replacement of ST to other types of activity. For this, three models were created: a single effect model for each type of activity (no adjustments for other activities, nor waking time), a partition model (each type of activity was adjusted for all other activities, but not waking time) and a substitution model (dropping ST from the model and introducing waking time). By doing so, in the substitution model, the regression coefficient of each type of activity represents the (independent) estimated change in $W_{\max } \mathrm{kg}^{-1}$ of replacing 1 hour of ST (the only type of activity not included in the model) by this activity. The associations in all models were adjusted for: age, education level, and type 2 diabetes, BMI, alcohol use, smoking status, cardio vascular disease, beta-blocker use, energy intake and mobility limitations. In these substitution model there was no indication for collinearity (all VIF <1.5, Pearson correlation coefficients between exposures: standing-LPA: 0.35, standing-HPA: 0.17, LPA-HPA:0.22).All analyses were performed using IBM SPSS Statistics for Windows, Version 22.0 (Armonk, NY, USA: IBM Corp).

\section{Results}

Compared with the excluded population $(\mathrm{N}=1,427)$, the included population of this study was generally healthier. For instance, the study population had a lower BMI and there were fewer participants with type 2 diabetes, a history of CVD and mobility limitations (data not tabulated). This was largely due to the exclusion criteria applied for the exercise test. Table 3.1 presents the characteristics of the total study population $(\mathrm{N}=2,024)$ and stratified by sex. The participants, of whom $49.3 \%$ were men, had a mean $\pm S D$ age of $59.7 \pm 8.1$ years. More than $95 \%$ of the study population provided 4 or more valid days of activPAL data with, on average, 15.7 hours of waking time. With $9.8 \pm 1.5$ hours, men spent on average one hour per day more in a sedentary position during waking hours than women. Men had a higher estimated $\mathrm{W}_{\max }$ than women: $2.62 \pm 0.60$ versus $2.02 \pm 0.52 \mathrm{~W} \mathrm{~kg}^{-1}$.

Figure 3.1 presents the proportion of the day spent sedentary, standing, in LPA and HPA for each (age specific) CRF category. Proportion of time per day spent in a seden 
tary position was $65.3 \%$ for men and $59.2 \%$ for women with low level of CRF. This was $60.0 \%$ for men and $54.4 \%$ for women with high CRF. Standing time was higher in men and lower in women with increasing CRF level. Both men and women in the highest CRF category spent more time in LPA and HPA compared to those with lower CRF level.

Table 3.1: Descriptive characteristics of the total study population, and according to sex

\begin{tabular}{|c|c|c|c|c|c|c|}
\hline & \multicolumn{2}{|c|}{$\begin{array}{l}\text { Total population } \\
(\mathrm{n}=2,024)\end{array}$} & \multicolumn{2}{|c|}{$\begin{array}{l}\text { Men } \\
(\mathrm{n}=997)\end{array}$} & \multicolumn{2}{|c|}{$\begin{array}{l}\text { Women } \\
(\mathrm{n}=1,027)\end{array}$} \\
\hline Age (years) & 59.7 & (8.1) & 60.9 & (7.9) & 58.6 & $(8.2)$ \\
\hline \multicolumn{7}{|l|}{ Education level (\%) } \\
\hline - $\quad$ high & 39.6 & & 43.5 & & 35.8 & \\
\hline - $\quad$ medium & 28.5 & & 29.0 & & 27.9 & \\
\hline$-\quad$ low & 31.9 & & 27.5 & & 36.2 & \\
\hline Smoking status (\% current smokers) & 12.1 & & 13.1 & & 11.1 & \\
\hline $\begin{array}{l}\text { Alcohol consumption } \\
\text { (\% high consumers) }\end{array}$ & 25.4 & & 23.1 & & 27.8 & \\
\hline Energy intake (kcal/day) & 2167.7 & $(596.4)$ & 2358.8 & $(618.5)$ & 1981.6 & $(509.9)$ \\
\hline Mobility limitation (\% with) & 17.1 & & 15.2 & & 18.9 & \\
\hline BMI (kg/m2) & 26.7 & $(4.3)$ & 27.4 & $(3.9)$ & 26.0 & $(4.5)$ \\
\hline (History of) cardiovascular disease (\%) & 13.9 & & 15.9 & & 12.0 & \\
\hline \multicolumn{7}{|l|}{ Glucose metabolism status (\%) } \\
\hline - $\quad$ normal glucose metabolism & 58.3 & & 47.3 & & 68.8 & \\
\hline - $\quad$ prediabetes & 15.5 & & 16.5 & & 14.4 & \\
\hline - $\quad$ type 2 diabetes & 25.3 & & 35.3 & & 15.6 & \\
\hline - $\quad$ type 1 diabetes or other & 0.9 & & 0.8 & & 1.2 & \\
\hline$\beta$-blockers (\%) & 15.8 & & 19.9 & & 11.9 & \\
\hline \multicolumn{7}{|l|}{ Number of valid days } \\
\hline$-\quad<4$ valid days $(\%)$ & 4.2 & & 4.8 & & 3.5 & \\
\hline - $\quad \geq 4$ valid days (\%) & 95.8 & & 95.2 & & 96.5 & \\
\hline Waking time (h/day) & 15.7 & $(0.9)$ & 15.8 & $(0.9)$ & 15.7 & $(0.9)$ \\
\hline Sedentary time (h/day) & 9.3 & (1.6) & 9.8 & (1.5) & 8.8 & $(1.6)$ \\
\hline Lower intensity physical activity (h/day) & 1.6 & $(0.5)$ & 1.7 & $(0.6)$ & 1.6 & $(0.5)$ \\
\hline $\begin{array}{l}\text { Higher intensity physical activity } \\
\text { (min/day) }\end{array}$ & 19.5 & [9.9-32.0] & 14.7 & {$[7.2-26.9]$} & 23.5 & {$[14.5-35.3]$} \\
\hline Sedentary breaks (N/day) & 37.6 & $(8.5)$ & 37.8 & $(9.0)$ & 37.4 & $(8.1)$ \\
\hline Average sedentary bout duration (min) & 11.1 & $(3.5)$ & 11.7 & (3.7) & 10.5 & $(3.1)$ \\
\hline Sedentary bouts $\geq 30 \mathrm{~min}$ (N/day) & 4.8 & $(1.5)$ & 5.1 & $(1.6)$ & 4.5 & $(1.4)$ \\
\hline CRF (Wmax) & 164.8 & $(48.3)$ & 191.3 & $(46.6)$ & 139.2 & $(34.1)$ \\
\hline CRF adjusted for body mass $\left(\mathrm{W}_{\max } \mathrm{kg}^{-1}\right)$ & 2.14 & $(0.58)$ & 2.26 & $(0.60)$ & 2.02 & $(0.52)$ \\
\hline
\end{tabular}

Values are means (SD), median [25-75\%], or percentages. BMI, body mass index; CRF, cardio-respiratory fitness; Wmax, estimated maximum work load (W). 


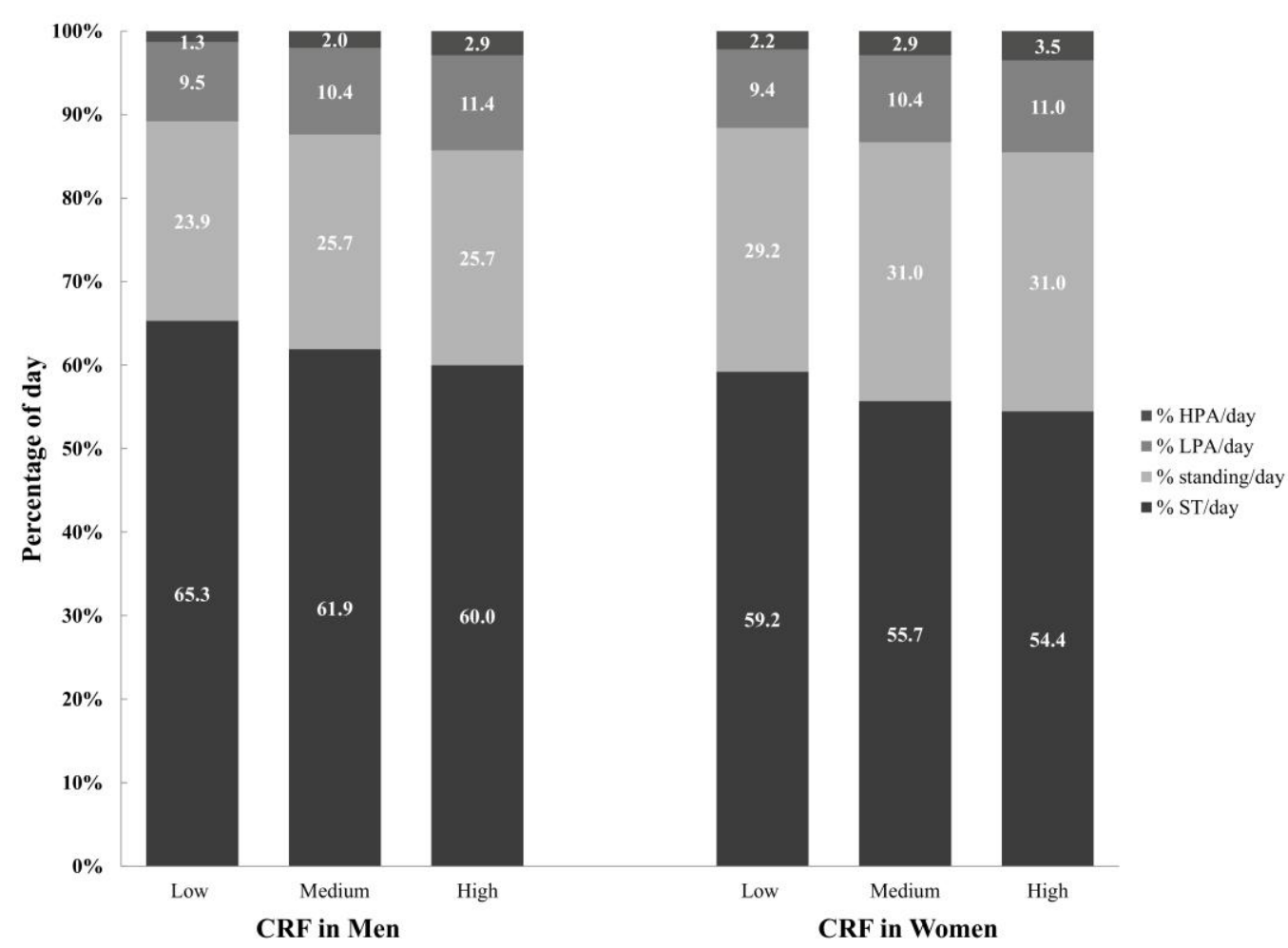

Figure 3.1: Percentage sedentary time (ST), standing time, lower intensity PA (LPA), and higher intensity PA (HPA) per day, according to CRF category (low, medium, and high) and sex.

Table 3.2 presents the waking time adjusted associations of ST and sedentary behaviour pattern variables with CRF $\left(\mathrm{W}_{\max } \mathrm{kg}^{-1}\right)$ stratified by sex. More ST per day was associated with lower CRF after adjustment for waking time, age, education level, type 2 diabetes and HPA (model 2): $\mathrm{B}_{\text {men }}=-0.05 \mathrm{~W} \mathrm{~kg}^{-1}(95 \% \mathrm{CI}=-0.07 ;-0.03)$ and $\mathrm{B}_{\text {women }}=-0.03 \mathrm{~W}$ $\mathrm{kg}^{-1}(-0.05 ;-0.01)$. As we adjusted for waking time and HPA, these regression coefficients should be interpreted as the associations of engaging 1 hour in ST instead of standing and/or LPA. After adjustment for BMI, alcohol use, smoking status, cardiovascular disease, beta-blocker use, energy intake, and mobility limitations (model 3) associations remained statistically significant. More sedentary breaks per day were associated with higher CRF independent of HPA and ST (model 2): $\mathrm{B}_{\text {men }}=0.04 \mathrm{~W} \mathrm{~kg}^{-1}$ $(0.01 ; 0.08)$ and $B_{\text {women }}=0.06 \mathrm{~W} \mathrm{~kg}^{-1}(0.02 ; 0.09)$. A longer average sedentary bout duration was associated with lower CRF independent of HPA and ST (model 2): $\mathrm{B}_{\text {men }}=-0.02$ $\mathrm{W} \mathrm{kg}^{-1}(-0.03 ;-0.01)$ and $\mathrm{B}_{\text {women }}=-0.02 \mathrm{~W} \mathrm{~kg}^{-1}(-0.03 ;-0.01)$. After adjustment for additional potential confounders (model 3 ) these associations became statistically nonsignificant. Associations between prolonged sedentary bouts and CRF were statistically sig- 
nificant in model 1 but this was no longer seen after additional adjustment for HPA and ST (model 2); $\mathrm{B}_{\text {men }}=-0.02 \mathrm{~W} \mathrm{~kg}^{-1}(-0.05 ; 0.01)$ and $\mathrm{B}_{\text {women }}=-0.03 \mathrm{~W} \mathrm{~kg}^{-1}(-0.06 ; 0.00)$.

Table 3.2: Associations of sedentary time and sedentary behavior pattern variables with estimated CRF (W kg -1) stratified by sex

\begin{tabular}{|c|c|c|c|c|c|c|c|c|}
\hline & \multicolumn{2}{|c|}{ Sedentary time } & \multicolumn{2}{|c|}{$\begin{array}{l}\text { Sedentary breaks } \\
\text { (10/day) }\end{array}$} & \multicolumn{2}{|c|}{$\begin{array}{l}\text { Average sedentary } \\
\text { bout duration } \\
\text { (min) }\end{array}$} & \multicolumn{2}{|c|}{$\begin{array}{l}\text { Prolonged sedentary } \\
\text { bouts (n/day) }\end{array}$} \\
\hline Men & B & $(95 \% \mathrm{CI})$ & B & $(95 \%$ CI $)$ & B & $(95 \% \mathrm{CI})$ & B & $(95 \%$ CI $)$ \\
\hline Model 1 & -0.08 & $(-0.10 ;-0.06)$ & 0.09 & $(0.05 ; 0.13)$ & -0.03 & $(-0.04 ;-0.02)$ & -0.07 & $(-0.09 ;-0.05)$ \\
\hline Model 2 & -0.05 & $(-0.07 ;-0.03)$ & 0.04 & $(0.01 ; 0.08)$ & -0.02 & $(-0.03 ;-0.01)$ & -0.02 & $(-0.05 ; 0.01)$ \\
\hline Model 3 & -0.03 & $(-0.05 ;-0.01)$ & 0.02 & $(-0.02 ; 0.05)$ & -0.01 & $(-0.02 ; 0.00)$ & -0.01 & $(-0.04 ; 0.02)$ \\
\hline Women & & & & & & & & \\
\hline Model 1 & -0.06 & $(-0.08 ;-0.04)$ & 0.08 & $(0.04 ; 0.11)$ & -0.03 & $(-0.04 ;-0.02)$ & -0.06 & $(-0.08 ;-0.04)$ \\
\hline Model 2 & -0.03 & $(-0.05 ;-0.01)$ & 0.06 & $(0.02 ; 0.09)$ & -0.02 & $(-0.03 ;-0.01)$ & -0.03 & $(-0.06 ; 0.00)$ \\
\hline Model 3 & -0.02 & $(-0.04 ; 0.00)$ & 0.02 & $(-0.01 ; 0.06)$ & -0.01 & $(-0.02 ; 0.00)$ & -0.01 & $(-0.04 ; 0.02)$ \\
\hline
\end{tabular}

Results are presented as unstandardized regression coefficients (B), with 95\% confidence interval (95\% CI). The associations in models 1 adjusted for: waking time, age, education level, and type 2 diabetes. Model 2 : model 1 + higher intensity physical activity for sedentary time; + higher intensity physical activity and sedentary time for sedentary breaks, mean bout duration and prolonged sedentary bouts. Model 3: model $2+$ BMI, alcohol use, smoking status, cardio vascular disease, beta-blocker use, energy intake and mobility limitations. Bold fonts indicate statistical significance $\mathrm{p}<0.05$.

Table 3.3 presents the waking time adjusted associations of LPA and HPA with CRF $\left(\mathrm{W}_{\max } \mathrm{kg}^{-1}\right)$ stratified by sex. More time spent in LPA and HPA were associated with higher CRF. Statistically significant associations between LPA and CRF were observed after adjustment for all potential confounders (model 3): $\mathrm{B}_{\text {men }}=0.12 \mathrm{~W} \mathrm{~kg}^{-1}(0.07 ; 0.17)$ and $B_{\text {women }}=0.12 \mathrm{~W} \mathrm{~kg}^{-1}(0.07 ; 0.18)$. Associations between HPA and CRF in models 3 were: $B_{\text {men }}=0.48 \mathrm{~W} \mathrm{~kg}^{-1}(0.38 ; 0.58)$ and $B_{\text {women }}=0.27 \mathrm{~W} \mathrm{~kg}^{-1}(0.18 ; 0.36)$. One may interpret these regression coefficients as the associations of engaging 1 hour in LPA and $\mathrm{HPA}$, respectively, as a replacement for engaging in an activity not included in the model.

Table 3.4 shows that each type of activity was associated with CRF in the single effect models (similar as in tables 2 and 3 but without adjustment for waking time). Further it shows that, when all activities were adjusted for each other (partition model), only LPA and HPA were associated with CRF. The substitution model shows the effect on CRF of the theoretical replacement of ST by other types of activity (standing, LPA and HPA). After adjustment for potential confounders, replacing 1 hour of ST with standing was not associated with higher CRF. Replacing 1 hour of ST with 1 hour of LPA was associ- 
ated with higher CRF: $\mathrm{B}_{\text {men }}=0.08 \mathrm{~W} \mathrm{~kg}^{-1}(0.03 ; 0.14)$ and $\mathrm{B}_{\text {women }}=0.10 \mathrm{~W} \mathrm{~kg}^{-1}(0.05 ; 0.15)$. Replacing 1 hour of ST with 1 hour of HPA was associated with higher CRF: $\mathrm{B}_{\text {men }}=0.49$ $\mathrm{W} \mathrm{kg}^{-1}(0.39 ; 0.59)$ and $\mathrm{B}_{\text {women }}=0.28 \mathrm{~W} \mathrm{~kg}^{-1}(0.10 ; 0.36)$.

Table 3.3: Associations of lower intensity activity and higher intensity physical with estimated CRF (W kg -1) stratified by sex

\begin{tabular}{lllll} 
& \multicolumn{2}{c}{$\begin{array}{l}\text { Lower intensity physical activity } \\
\text { (h/day) }\end{array}$} & \multicolumn{2}{c}{ Higher intensity physical activity (h/day) } \\
Men & \multicolumn{2}{c}{ B } & $(95 \%$ CI $)$ & \multicolumn{2}{c}{ B } & $(95 \%$ CI $)$ \\
\hline Model 1 & 0.22 & $(0.16 ; 0.27)$ & 0.68 & $(0.58 ; 0.79)$ \\
Model 2 & n.a. & & 0.61 & $(0.50 ; 0.72)$ \\
Model 3 & 0.12 & $(0.07 ; 0.17)$ & 0.48 & $(0.38 ; 0.58)$ \\
Women & & & & \\
\hline Model 1 & $\mathbf{0 . 2 0}$ & $(0.14 ; 0.25)$ & 0.45 & $(0.36 ; 0.54)$ \\
Model 2 & n.a. & & 0.40 & $(0.31 ; 0.50)$ \\
Model 3 & 0.12 & $(0.07 ; 0.18)$ & 0.27 & $(0.18 ; 0.36)$ \\
\hline
\end{tabular}

Results are presented as unstandardized regression coefficients (B), with 95\% confidence interval (95\% CI). The associations in models 1 adjusted for: waking time, age, education level, and type 2 diabetes. Model 2: model $1+$ sedentary time. n.a.: No additional adjustment for other type(s) of activity in the model for lower intensity physical activity due to collinearity. Model 3: model 2 + BMI, alcohol use, smoking status, cardio vascular disease, beta-blocker use, energy intake and mobility limitations. Bold fonts indicate statistical significance $\mathrm{p}<0.05$.

Table 3.4: Single, partition, and isotemporal substitution models examining the associations of sedentary time, standing time, lower- and higher intensity physical actvity with $\mathrm{CRF}$ and the theoretical replacement of 1 hour sedentary time with other activities, stratified by sex

\begin{tabular}{|c|c|c|c|c|c|c|c|c|}
\hline & \multicolumn{2}{|c|}{ Sedentary time } & \multicolumn{2}{|c|}{ Standing } & \multicolumn{2}{|c|}{$\begin{array}{l}\text { Lower intensity } \\
\text { physical activity }\end{array}$} & \multicolumn{2}{|c|}{$\begin{array}{l}\text { Higher intensity } \\
\text { physical actvity }\end{array}$} \\
\hline Men & B & $(95 \% \mathrm{CI})$ & B & $(95 \% \mathrm{CI})$ & B & $(95 \% \mathrm{CI})$ & B & $(95 \% \mathrm{CI})$ \\
\hline Model A & -0.04 & $(-0.11 ;-0.06)$ & 0.03 & $(0.01 ; 0.06)$ & 0.14 & $(0.09 ; 0.19)$ & 0.53 & $(0.43 ; 0.63)$ \\
\hline Model B & 0.02 & $(-0.0 ; 0.06)$ & 0.03 & $(-0.00 ; 0.07)$ & 0.11 & $(0.05 ; 0.17)$ & 0.52 & $(0.41 ; 0.62)$ \\
\hline Model C & & Dropped & 0.01 & $(-0.02 ; 0.04)$ & 0.08 & $(0.03 ; 0.14)$ & 0.49 & $(0.39 ; 0.59)$ \\
\hline Women & & & & & & & & \\
\hline Model A & -0.02 & $(-0.04 ;-0.01)$ & 0.02 & $(0.00 ; 0.04)$ & 0.14 & $(0.09 ; 0.19)$ & 0.32 & $(0.23 ; 0.40)$ \\
\hline Model B & 0.03 & $(0.00 ; 0.06)$ & 0.03 & $(0.00 ; 0.06)$ & 0.13 & $(0.08 ; 0.20)$ & 0.31 & $(0.22 ; 0.40)$ \\
\hline Model C & & Dropped & -0.00 & $(-0.02 ; 0.02)$ & 0.10 & $(0.05 ; 0.16)$ & 0.28 & $(0.19 ; 0.36)$ \\
\hline
\end{tabular}

The associations in the all models were adjusted for age, education level, and type 2 diabetes, BMI, alcohol use, smoking status, cardio vascular disease, beta-blocker use, energy intake and mobility limitations. Further: In model A (single effect model): no adjustment for other type of activity nor for wear time. In model B (partition model), each activity was adjusted for all other types of activity, no adjustment for wear time. In model C (substitution model), sedentary time was dropped from the model, and additionally adjusted for wear time. Bold fonts indicate statistical significance $p<0.05$. 
Results were similar when analyses were repeated after excluding participants with $<4$ valid days of activPAL measurement (data not shown). Additionally, results were similar after excluding participants where Wmax was calculated from completed ergometer tests (data not shown).

\section{Discussion}

This study is one of the first to evaluate the mutually independent associations of objectively measured ST, sedentary behaviour patterns, LPA, and HPA with CRF. Our results show that more ST per day was associated with lower CRF, independent from HPA. Sedentary breaks and average sedentary bout duration were associated also with CRF. However, these associations were attenuated and no longer statistically significant in the fully adjusted models. Further, both daily LPA and HPA were positively associated with CRF, independent of potential confounders. The theoretical replacement of ST with HPA or with LPA was associated with higher CRF. The greatest estimated change in CRF was observed when ST was replaced with HPA.

In our study, one additional hour of ST corresponded with, on average, $\sim 1.2 \%$ lower $\mathrm{W}_{\max } \mathrm{kg}^{-1}$ for men and $\sim 0.9 \%$ lower $\mathrm{W}_{\max } \mathrm{kg}^{-1}$ for women. The strength of these associations is clearly less than strength of the association observed between LPA and HPA with CRF; reducing one hour of ST approximately resembles an increase of five minutes of HPA. However, small improvements in CRF have been associated with reduced risk for cardiovascular disease and mortality in particularly the least-fit part of the adult population. ${ }^{28}$ Therefore, reductions in ST may improve CRF, which could be beneficial for health. This conclusion is supported by the results from the isotemporal substitution analyses that show that replacing ST with LPA was associated with higher CRF (independent of time engaged in standing and HPA). Of note, replacing ST with HPA was associated with a greater estimated change in CRF. Consequently, engaging in HPA is most important for CRF than increasing time spent with LPA. However, for many people (elderly or those with functional limitations) replacing ST by LPA may be more feasible than replacing ST by HPA.

To our knowledge, only two studies have previously reported on associations between objectively measured ST and CRF in adults. ${ }^{18,29}$ In the study by Kulinski et al., a small, but statistically significant inverse association between objectively measured ST and CRF was reported in a population aged 12-49 years, after adjustment for MVPA. ${ }^{18}$ In the study by Prince et al. an inverse association between ST and CRF was reported in a post-cardiac rehabilitation population. ${ }^{29}$ Both studies are in line with our findings. Several other studies have evaluated the association between ST and CRF using selfreported measures of ST. Results from these studies were inconsistent. Tucker et al. 
reported that female frequent TV viewers had a lower $\mathrm{CRF}$, but after adjustment for PA and BMI this effect was attenuated and no longer statistically significant. ${ }^{17}$ A large scale population based study by Eriksen et al. reported inverse associations between ST and CRF in participants who reported low levels of physical activity, while no association between ST and CRF was reported in those who were classified as being moderately or vigorously active. ${ }^{16}$ Lastly, Barlow et al. found a detrimental association between more self-reported sitting time and CRF in women independent of levels of PA. ${ }^{30}$

This study is, to the best of our knowledge, the first to examine pattern variables of sedentary behaviour with CRF. Sedentary breaks have previously been associated with improved cardio-metabolic health in some studies. ${ }^{14,15}$ In our study, an association was observed between sedentary breaks and CRF independent of ST and HPA, but not independent of health related variables in model 3. Similar results were observed for average sedentary bout duration. Prolonged sedentary bouts lasting $\geq 30$ minutes were not associated with CRF independent of HPA and ST. Although it has been suggested that long uninterrupted periods of ST are detrimental for health, ${ }^{13,14}$ it is unknown at what duration a prolonged sedentary bout becomes detrimental. Possibly this is not at 30 minutes, but after 60 or 90 minutes or even longer. Based on our results, total ST seems to be more important for CRF than SB patterns. The mechanisms through which sedentary behaviour affects CRF are not yet fully understood. Partly it could be explained by vascular changes in response to sedentary behaviour. The pathways through which these changes occur, appear to be distinct from pathways that are involved in vascular changes in response to PA. ${ }^{31}$ Clearly, more research in this area is warranted to further investigate how sedentary behaviour affects CRF.

Our finding that LPA and HPA were strongly associated with CRF elaborates on the existing body of evidence linking PA to CRF.6,7 Mechanisms through which HPA improves CRF have been studied comprehensively and include vascular, muscular, and respiratory adaptations. ${ }^{32}$ These responses are found to be largest when PA is of higher intensity and/or longer duration. In our study, one additional hour of HPA per day corresponded with, on average, $\sim 21 \%$ higher $W_{\max } \mathrm{kg}^{-1}$ for men and $\sim 13 \%$ higher $W_{\max }$ $\mathrm{kg}^{-1}$ for women. Similar amounts have been associated with reduced mortality risk. ${ }^{4}$ The difference between men and women in our study may be explained by a larger range in $W_{\max }$ for men compared with women, as men are physiologically capable of achieving higher levels of $W_{\max }$.

A strength of this study was the use of a posture based accelerometer in a large sample of adults. The activPAL3 has been found to accurately measure (patterns of) sedentary behaviour. $33-35$ Therefore, our estimations of ST were probably better than those in previous studies using other types of accelerometers. Further, CRF was estimated from a 
sub-maximal exercise protocol incorporating both HR and RPE. Extrapolating submaximal values of RPE, similarly as HR, has been shown to be an alternative and valid method to estimate CRF. ${ }^{36}$ By incorporating RPE in addition to HR, estimates of CRF were also obtained from individuals who otherwise would have been excluded from exercise testing due to medication use or medical conditions. Nevertheless, exclusion for exercise testing based on medical condition has introduced selection bias in this study, though to a lesser extent than in many other epidemiological studies measuring CRF. It should be noted that the analyses to compare $W_{\operatorname{maxHR}}$ with $W_{\text {maxRPE }}$ (see supplement) were only performed in a subset of the Maastricht Study population. Furthermore, the use of a sub-maximal exercise test instead of a maximal exercise test to estimate Wmax may be considered as a limitation, since this may have resulted in underor overestimation of actual Wmax. Nevertheless, high correlations ( $r=0.76-0.98)$ between estimated CRF and measured CRF have been reported in previous studies. ${ }^{37}$ Further, the associations in this study were adjusted for a series of potential confounders. As The Maastricht Study is enriched with participants with type 2 diabetes, adjustments were made for type 2 diabetes in the associations of models 1 . However, type 2 diabetes may lie in the pathway between sedentary behaviour and CRF, as ST has previously been associated with increased odds for T2DM in this population. ${ }^{38}$ Consequently, these adjustments may have resulted in over adjustment bias. Over adjustment may also be an issue in the fully adjusted models (models 3). For instance, sedentary behaviour has been associated with increased BMI and increased risk for CVD, which both have been associated with lower CRF. Consequently, the strength of the associations as reported in this study may actually be greater.

Some other limitations should be considered as well. Most importantly, the crosssectional design of the study requires caution with regard to causal inferences. In addition, at least one valid day of accelerometer wear time was considered sufficient in this study, but one day may not be representative for habitual behaviour. However, $95 \%$ of the study population provided at least 4 valid days of accelerometer data and results were similar when participants with less than 4 valid days of wear time were excluded from analyses. Further, LPA and HPA was based on step frequency which may be less precise to determine intensity levels compared with estimations of LPA and HPA based on acceleration data. However, we used a step frequency of $>\sim 100$ steps/minute which has been reported to correspond to a MET score of $\sim 3.0$ (a frequently used cutoff value for moderate to vigorous PA). ${ }^{39}$ Finally, our study population consisted of a relatively healthy population of predominantly Caucasians from European descent with well controlled participants with type 2 diabetes. Therefore, generalizability of our results may be limited. 
In conclusion, more daily ST appeared to be modestly associated with lower CRF, independent of HPA. Both LPA and HPA were associated with higher CRF. Replacing ST with LPA was associated with a positive estimated change in CRF, which is particularly helpful for people who are unable to engage in HPA. Further, replacing ST with HPA was associated with greatest estimated change in CRF. Therefore, engaging regularly in HPA appears to be the best strategy to improve CRF. 


\section{References}

1. Lee DC, Artero EG, Sui X, Blair SN. Review: Mortality trends in the general population: the importance of cardiorespiratory fitness. J. Psychopharmacol. 2010;24(4 suppl):27-35.

2. Blair SN, Kampert JB, Kohl HW, Barlow CE, Macera CA, Paffenbarger RS, Gibbons LW. Influences of cardiorespiratory fitness and other precursors on cardiovascular disease and all-cause mortality in men and women. JAMA 1996;276(3):205-210.

3. Katzmarzyk PT, Church TS, Blair SN. Cardiorespiratory fitness attenuates the effects of the metabolic syndrome on all-cause and cardiovascular disease mortality in men. Arch. Intern. Med. 2004;164(10):1092-1097.

4. Kodama S, Saito K, Tanaka S, Maki M, Yachi Y, Asumi M, Sugawara A, Totsuka K, Shimano H, Ohashi Y. Cardiorespiratory fitness as a quantitative predictor of all-cause mortality and cardiovascular events in healthy men and women: a meta-analysis. JAMA 2009;301(19):2024-2035.

5. Bouchard C, Rankinen T. Individual differences in response to regular physical activity. Med. Sci. Sports Exerc. 2001;33(6 Suppl):S446-51; discussion S452-3.

6. Lin X, Zhang X, Guo J, Roberts CK, McKenzie S, Wu WC, Liu S, Song Y. Effects of Exercise Training on Cardiorespiratory Fitness and Biomarkers of Cardiometabolic Health: A Systematic Review and Meta-Analysis of Randomized Controlled Trials. J Am Heart Assoc 2015;4(7):e002014.

7. Laukkanen JA, Laaksonen D, Lakka TA, Savonen K, Rauramaa R, Mäkikallio T, Kurl S. Determinants of cardiorespiratory fitness in men aged 42 to 60 years with and without cardiovascular disease. Am. J. Cardiol. 2009;103(11):1598-1604.

8. Tucker JM, Welk GJ, Beyler NK. Physical activity in US adults: compliance with the physical activity guidelines for Americans. Am. J. Prev. Med. 2011;40(4):454-461.

9. Owen N, Healy GN, Matthews CE, Dunstan DW. Too much sitting: the population health science of sedentary behavior. Exerc. Sport Sci. Rev. 2010;38(3):105-13.

10. Sedentary Behaviour Research Network. (2102). Letter to the editor: standardized use of the terms "sedentary" and "sedentary behaviours". Appl. Physiol. Nutr. Metab;37:540-542.

11. Brocklebank LA, Falconer CL, Page AS, Perry R, Cooper AR. Accelerometer-measured sedentary time and cardiometabolic biomarkers: A systematic review. Prev. Med. 2015;76:92-102.

12. Wilmot EG, Edwardson CL, Achana FA, Davies MJ, Gorely T, Gray LJ, Khunti K, Yates T, Biddle SJ. Sedentary time in adults and the association with diabetes, cardiovascular disease and death: systematic review and meta-analysis. Diabetologia 2012;55(11):2895-2905.

13. Duvivier BM, Schaper NC, Bremers MA, van Crombrugge G, Menheere PP, Kars M, Savelberg HH. Minimal Intensity Physical Activity (Standing and Walking) of Longer Duration Improves Insulin Action and Plasma Lipids More than Shorter Periods of Moderate to Vigorous Exercise (Cycling) in Sedentary Subjects When Energy Expenditure Is Comparable. PLoS ONE 2013;8(2):e55542.

14. Dunstan DW, Kingwell BA, Larsen R, Healy GN, Cerin E, Hamilton MT, Shaw JE, Bertovic DA, Zimmet PZ, Salmon J. Breaking up prolonged sitting reduces postprandial glucose and insulin responses. Diabetes Care 2012;35(5):976-983.

15. Healy GN, Dunstan DW, Salmon J, Cerin E, Shaw JE, Zimmet PZ, Owen N. Breaks in sedentary time beneficial associations with metabolic risk. Diabetes Care 2008;31(4):661-666.

16. Eriksen L, Grønbæk M, Helge J, Tolstrup J. Cardiorespiratory fitness in 16025 adults aged 18-91 years and associations with physical activity and sitting time. Scand. J. Med. Sci. Sports 2015.

17. Tucker LA, Arens PJ, LeCheminant JD, Bailey BW. Television viewing time and measured cardiorespiratory fitness in adult women. Am. J. Health Promot. 2015;29(5):285-290.

18. Kulinski JP, Khera A, Ayers CR, Das SR, de Lemos JA, Blair SN, Berry JD. Association between cardiorespiratory fitness and accelerometer-derived physical activity and sedentary time in the general population. Mayo Clin. Proc. 2014;89(8):1063-1071.

19. Schram MT, Sep SJ, van der Kallen CJ, Dagnelie PC, Koster A, Schaper N, Henry RM, Stehouwer $\mathrm{CD}$. The Maastricht Study: an extensive phenotyping study on determinants of type 2 diabetes, its complications and its comorbidities. Eur. J. Epidemiol. 2014;29(6):439-51.

20. van der Berg JD, Willems PJ, van der Velde JHPM, Savelberg HHCM, Schaper NC, Schram MT, Sep SJ, Dagnelie PC, Bosma H, Stehouwer CDA. Identifying waking time in 24-h accelerometry data in adults using an automated algorithm. J. Sports Sci. 2016;34(19):1867-73. 
21. Tudor-Locke C, Rowe DA. Using cadence to study free-living ambulatory behaviour. Sports Med. 2012;42(5):381-398.

22. Storer TW, Davis JA, Caiozzo VJ. Accurate prediction of VO2max in cycle ergometry. Med. Sci. Sports Exerc. 1990;22(5):704-12.

23. Bovens A, Van Baak M, Vrencken J, Wijnen J, Saris W, Verstappen F. Maximal aerobic power in cycle ergometry in middle-aged men and women, active in sports, in relation to age and physical activity. Int. J. Sports Med. 1993;14(2):66-71.

24. Sartor F, Vernillo G, de Morree HM, Bonomi AG, La Torre A, Kubis H-P, Veicsteinas A. Estimation of maximal oxygen uptake via submaximal exercise testing in sports, clinical, and home settings. Sports Med. 2013;43(9):865-873.

25. Health. Council of the Netherlands. Guidelines for a healthy diet 2006. The Hague: Health Council of the Netherlands, 2006; publication no. 2006/21.

26. Aaronson NK, Muller M, Cohen PD, Essink-Bot M-L, Fekkes M, Sanderman R, Sprangers MA, te Velde A, Verrips E. Translation, validation, and norming of the Dutch language version of the SF36 Health Survey in community and chronic disease populations. J. Clin. Epidemiol. 1998;51(11):1055-1068.

27. World Health Organization. Definition and Diagnosis of Diabetes Mellitus and Intermediate Hyperglycemia. 2006.

28. Blair SN, Connelly JC. How much physical activity should we do? The case for moderate amounts and intensities of physical activity. Res. Q. Exerc. Sport 1996;67(2):193-205.

29. Prince SA, Blanchard CM, Grace SL, Reid RD. Objectively-measured sedentary time and its association with markers of cardiometabolic health and fitness among cardiac rehabilitation graduates. Eur J Prev Cardiol 2015;23:818-825.

30. Barlow CE, Shuval K, Balasubramanian BA, Kendzor DE, Gabriel KP. Sitting Time, Physical Activity and Cardiorespiratory Fitness: CCLS Cohort. J. Phys. Act. Health 2016;13(1):17-23.

31. Thijssen DH, Green DJ, Hopman MT. Blood vessel remodeling and physical inactivity in humans. J. Appl. Physiol. 2011;111(6):1836-1845.

32. McArdle WD, Katch FI, Katch VL. Exercise physiology: nutrition, energy, and human performance Lippincott Williams \& Wilkins, 2010.

33. Kozey-Keadle S, Libertine A, Lyden K, Staudenmayer J, Freedson PS. Validation of wearable monitors for assessing sedentary behavior. Med. Sci. Sports Exerc. 2011;43(8):1561-7.

34. Lyden K, Kozey-Keadle SL, Staudenmayer JW, Freedson PS. Validity of two wearable monitors to estimate breaks from sedentary time. Med. Sci. Sports Exerc. 2012;44(11):2243.

35. Berendsen BAJ, Hendriks MRC, Meijer K, Plasqui G, Schaper NC, Savelberg HHCM. Which activity monitor to use? Validity, reproducibility and user friendliness of three activity monitors. BMC Public Health 2014;14(749).

36. Coquart JB, Garcin M, Parfitt G, Tourny-Chollet C, Eston RG. Prediction of maximal or peak oxygen uptake from ratings of perceived exertion. Sports Med. 2014;44(5):563-578.

37. Akalan C, Robergs RA, Kravitz L. Prediction of VO2max from an individualized submaximal cycle ergometer protocol. J Exerc Physiol Online 2008;11(2):1-17.

38. van der Berg JD, Stehouwer CD, Bosma H, van der Velde JH, Willems PJ, Savelberg HH, Schram MT, Sep SJ, van der Kallen CJ, Henry RM. Associations of total amount and patterns of sedentary behaviour with type 2 diabetes and the metabolic syndrome: The Maastricht Study. Diabetologia 2016;59(4):709-718.

39. Tudor-Locke C, Craig CL, Brown WJ, Clemes SA, De Cocker K, Giles-Corti B, Hatano Y, Inoue S, Matsudo SM, Mutrie N, Oppert JM, Rowe DA, Schmidt MD, Schofield GM, Spence JC, Teixeira PJ, Tully MA, Blair SN. How many steps/day are enough? For adults. Int J Behav Nutr Phys Act 2011;8:79. 
Supplement to chapter 3 


\section{Supplement S3.1}

To compare estimated Wmax based on HR (WmaxHR85\%) and Wmax based on RPE (WmaxRPE17) the difference in Wmax between the two methods was calculated. This was done only with those participants that attained both $85 \%$ of HRmax and an RPE of 17 at test end $(n=317)$. Mean difference was calculated and presented as $95 \%$ limits of agreement (Mean difference in $\mathrm{W} \pm 2 \mathrm{SD}$ ) and plotted using the Bland-Altman technique (see figure): $15.6 \pm 37.4 \mathrm{~W}$. Additionally, intraclass correlation coefficient (ICC) with 95\% confidence interval was calculated: 0.89 [0.86-0.91].

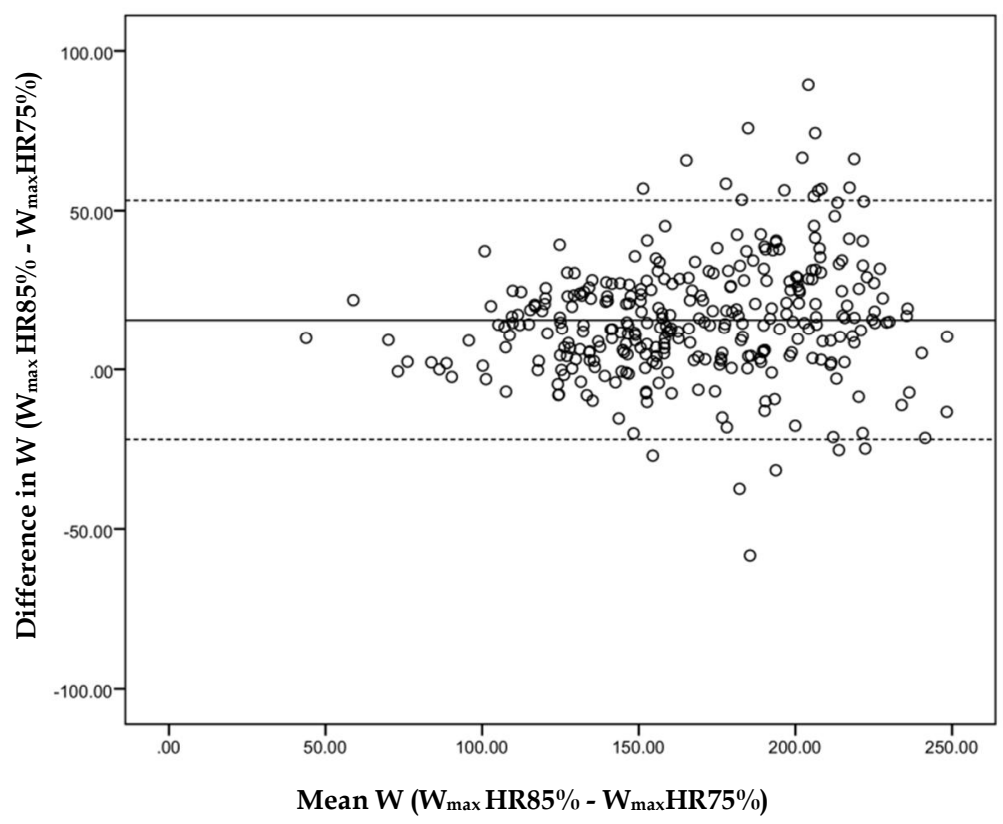

Figure S3.1: Bland Atman-plot of WmaxHR85\% versus WmaxRPE17. Solid line indicates bias, dashed lines indicate $95 \%$ limits of agreement. 


\section{Supplement S3.2}

\section{Comparison of HR}

First we selected participants that achieved $85 \%$ of $\operatorname{HR}_{\max }(n=1,728)$ and estimated $W_{\text {Max }}$ $\left(=\mathrm{W}_{\operatorname{maxHR} 85 \%}\right)$ using individual linear regression models. Next we deleted all stages with a $H R$ value $\geq 81 \%$ of $H_{\max }$.

After removal of these data there were 342 participants that now had $80 \pm 1 \%$ of $H R_{\max }$ as highest $H R$ value. We estimated $W_{\max }$ again but this time using only $H R$ values up to $80 \pm 1 \%$ of $\mathrm{HR}_{\max }\left(=\mathrm{W}_{\max 80 \% \mathrm{HR}}\right)$. NB: The small $\mathrm{N}$ in the new group is due to stepwise increase of $\mathrm{HR}$ values (one HR value per 2-minute stage).

Moreover, we deleted all stages with a $H R$ value $\geq 76 \%$ of $\mathrm{HR}_{\max }$. There were 354 participants with $75 \pm 1 \%$ of $\mathrm{HR}_{\max }$ as new highest $H R$ value. We estimated $W_{\max }$ again in this group, this time using only $\mathrm{HR}$ values up to $75 \pm 1 \%$ of $\mathrm{HR}_{\max }\left(=\mathrm{W}_{\operatorname{maxHR} 75 \%}\right)$.

Finally, we deleted all stages with a $H R$ value $\geq 71 \%$ of $H_{\max }$. There were 338 participants with $70 \pm 1 \%$ of $\mathrm{HR}_{\max }$ as new highest $H R$ value. We estimated $W_{\max }$ again in this group, now using only $\mathrm{HR}$ values up to $70 \pm 1 \%$ of $\mathrm{HR}_{\max }\left(=\mathrm{W}_{\max 70 \% \mathrm{HR}}\right)$.

Differences of $W_{\operatorname{maxHR} 85 \%}$ vs. $W_{\operatorname{maxHR} 8 \%}, W_{\operatorname{maxHR} 85 \%}$ vs. $W_{\operatorname{maxHR75}} \%$ and $W_{\operatorname{maxHR} 85} \%$ vs. $\mathrm{W}_{\text {maxHR70 }} \%$ were calculated and presented as 95\% limits of agreement (Mean difference \pm 2SD). Additionally, (two-way mixed effects) intraclass correlation coefficients (ICC) with $95 \%$ confidence intervals were calculated to quantify consistency between estimates.

Table S3.1: Comparison of estimated Wmax from HR85\% with estimates of Wmax from lower HR values

95\% Limits of Agreement

ICC $[95 \% \mathrm{CI}]$

$\begin{array}{ccc}\mathrm{W}_{\operatorname{maxHR} 85 \%}-\mathrm{W}_{\operatorname{maxHR} 80 \%} & -9.99 \pm 21.90 \mathrm{~W} & 0.97[0.96-0.97] \\ \mathrm{W}_{\operatorname{maxHR} 85 \%}-\mathrm{W}_{\operatorname{maxHR75} \%} & -16.12 \pm 40.54 \mathrm{~W} & 0.90[0.87-0.92] \\ \mathrm{W}_{\operatorname{maxHR} 85 \%-\mathrm{W}_{\operatorname{maxHR70} \%}} & -29.43 \pm 82.08 \mathrm{~W} & 0.68[0.61-0.73]\end{array}$




\section{Comparison of RPE}

First we selected the participants that achieved RPE $17(n=788)$ and estimated $W_{\max }$ $\left(=\mathrm{W}_{\text {maxRPE17 }}\right)$ using individual linear regression models (as in the figure on page 1$)$. Next we deleted all stages with $\mathrm{RPE} \geq 17$.

After removal of these data there were 196 participants that now had RPE 16 as highest Borg-score. We estimated $W_{\max }$ again but now only using RPE values up 16 $\left(=\mathrm{W}_{\text {maxRPE16) }}\right.$.

Moreover, we deleted all stages with a RPE value $\geq 16$. There were 169 participants with RPE 15 as new highest Borg-score. We estimated $W_{\max }$ again in this group, now only using RPE values up to 15 (= $\mathrm{W}_{\text {maxRPE15) }}$.

Finally, we deleted all stages with a RPE value $\geq 15$. There were 202 participants with RPE 14 as new highest Borg-score. We estimated $W_{\max }$ again in this group, now only using RPE values up to $14\left(=\mathrm{W}_{\text {maxRPE14 }}\right)$.

Differences of $W_{\text {maxRPE17VS. }} W_{\text {maxRPE16, }} W_{\text {maxRPE17VS. }} W_{\text {maxRPE15 }} W_{\text {maxRPE17. }} \cdot W_{\text {maxRPE14 }}$ were calculated and presented as $95 \%$ limits of agreement (Mean difference \pm 2 SD). Additionally, intraclass correlation coefficients (two-way mixed effects) were calculated to quantify consistency between estimates.

Table S3.2: Comparison of estimated Wmax from RPE17 with estimates of Wmax from lower RPE values

\section{5\% Limits of Agreement $\quad$ ICC $[95 \% \mathrm{CI}]$}

$\begin{array}{ccc}\mathrm{W}_{\text {maxRPE17 }}-\mathrm{W}_{\text {maxRPE16 }} & 0.02 \pm 22.30 \mathrm{~W} & 0.94[0.92-0.95] \\ \mathrm{W}_{\text {maxRPE17 }}-\mathrm{W}_{\text {maxRPE15 }} & -2.36 \pm 32.31 \mathrm{~W} & 0.93[0.91-0.93] \\ \mathrm{W}_{\text {maxRPE17 }}-\mathrm{W}_{\text {maxRPE14 }} & -3.45 \pm 60.11 \mathrm{~W} & 0.76[0.70-0.81]\end{array}$




\section{Chapter 4}

Moderate activity and fitness, not sedentary time, are independently associated with cardio-metabolic risk in U.S. adults aged 18-49

\section{- NHANES -}

Jeroen HPM van der Velde, Hans HCM Savelberg, Nicolaas C Schaper, Annemarie Koster 


\section{Abstract}

This cross-sectional study is one of the first to examine and compare the independent associations of objectively measured sedentary time, moderate to vigorous physical activity (MVPA) and fitness with cardio-metabolic risk factors. We studied 543 men and women (aged 18-49 years) from the NHANES 2003-2004 survey. Sedentary time and MVPA were measured by accelerometry. Fitness was assessed with a submaximal treadmill test. Cardio-metabolic risk factors included: waist circumference (WC), BMI, blood pressure, fasting glucose, HDL- and non HDL cholesterol, triglycerides (TG), and C-reactive protein (CRP). Sedentary time, MVPA and fitness were used as predictors for the cardio-metabolic outcomes in a multiple regression analysis. Standardized regression coefficients were computed. Results show that sedentary time was associated with HDL-cholesterol $(\beta=-0.080, p=0.05)$ and TG $(\beta=0.080, p=0.03)$. These results became non-significant after adjustment for MVPA and fitness. MVPA was associated with WC $(\beta=-0.226)$, BMI $(\beta=-0.239)$, TG $(\beta=-0.108)$ and HDL-cholesterol $(\beta=$ 0.144) (all $\mathrm{p}<0.05$ ). These results remained significant after adjustment for sedentary time and fitness. Fitness was associated with WC $(\beta=-0.287)$, BMI $(\beta=-0.266)$, systolic blood pressure $(\beta=-0.159)$, TG $(\beta=-0.092)$, and CRP $(\beta=-0.130)$ (all $p<0.05)$. After adjustment for sedentary time and MVPA these results remained significant. These differences in relative importance of sedentary time, MVPA and fitness on cardiometabolic-risk are important in the design of prevention programs. In this population, the strength of the associations between MVPA and fitness with cardio-metabolic markers appeared to be similar; both MVPA and fitness showed independent associations with cardio-metabolic risk factors. In contrast, sedentary time showed no independent associations with cardio-metabolic risk after correction for fitness and MVPA. 


\section{Introduction}

Physical activity (PA) is effective in the prevention of cardiovascular disease (CVD) by improving cardio-metabolic risk factors such as hypertension, elevated LDL-cholesterol levels and increased inflammatory markers. ${ }^{1,2}$ Consequently, current guidelines for PA advocate to spend at least 150 min per week in activities with a moderate or high intensity, known as moderate to vigorous physical activity (MVPA). ${ }^{3}$ Although, MVPA is usually only a small part of a person's total PA, the majority of research on PA and health outcomes focuses on this part of the PA spectrum. ${ }^{4}$ More recent studies have examined the association between sedentary activities and health outcomes. Sedentary activities refer to activities that do not, or only marginally increase energy expenditure above the resting level and include activities such as sitting, lying down, and watching television. ${ }^{5}$ Higher sedentary time has been associated with increased risk of metabolic syndrome and increased levels of cardio-metabolic risk factors, independent of the amount of MVPA. 6,7 Even when PA guidelines are met, sitting for prolonged periods can still compromise metabolic health. ${ }^{8,9}$ Nevertheless, in Western society increasingly more time is spent in sedentary activities and this trend is expected to continue given the increasing changes in private and public environments. ${ }^{9}$

Cardiorespiratory fitness (henceforward fitness) is another important determinant of cardio-metabolic health as it is associated with a lower risk for metabolic syndrome, ${ }^{10}$ and lower CVD mortality. ${ }^{11}$ Several studies examined the relative importance of PA and fitness as predictors for CVD or (CVD) mortality. ${ }^{12-15}$ These studies found that fitness was more strongly related to CVD than MVPA. However these studies did not include sedentary time in their analyses; another limitation was the use of self-reported measures for PA.

In a cross-sectional study using NHANES 2003-2004 data we examined the strength of the associations between objectively measured sedentary time, MVPA and cardiorespiratory fitness with several cardio-metabolic risk factors: Body mass Index (BMI), waist circumference (WC), systolic and diastolic blood pressure (SBP and DBP), fasting glucose, HDL- and non HDL cholesterol, triglycerides (TG), and C-reactive protein (CRP) and metabolic syndrome. Obtaining insight in the relative importance of sedentary time, MVPA and fitness on these cardio-metabolic-risk factors will contribute to designing more adequate prevention programs. 


\section{Experimental Section}

\section{Study Population}

This study used data from the National Health and Nutrition Examination Survey (NHANES) 2003-2004. NHANES is a cross-sectional study using a complex and multistage design in order to obtain a representative sample for the US population. The survey consists of a household interview by trained professionals and an examination in mobile examination centres. Data, details of methods and procedures are found on the NHANES website. ${ }^{16}$ The study was conducted in accordance with the Declaration of Helsinki, and the protocol was approved by The National Center for Health Statistics Ethics Review Board. Written informed consent was obtained from all participants. Only participants aged from 12-49 years were eligible to perform the submaximal exercise test $(n=2809)$. From those, 2244 participants were selected with one or more valid days of accelerometry data. A valid day was defined as a day when the accelerometer was worn for ten hours or more. For the current analysis only participants aged $\geq 18$ years were included $(n=1198)$. Further, only participants with fasting blood samples were selected $(n=583$ ). Collection of fasting blood samples was performed in a randomly chosen subgroup. Subjects with one or more missing variables of cardiometabolic outcomes or confounders (BMI, WC, blood pressure, glucose, cholesterol, TG, CRP, health status and smoking) were excluded $(n=27)$. As a final step 13 people with fasting glucose $>7 \mathrm{mmol} / \mathrm{L}$ were excluded, to eliminate participants with diabetes resulting in 543 participants used in the current analysis.

\section{Accelerometry}

Physical activity was objectively measured using the uniaxial ActiGraph AM-7164 (ActiGraph, Ft. Walton Beach, FL, USA). This accelerometer was placed on an elastic belt and worn on the right hip. All participants were instructed to wear the accelerometer for seven consecutive days and to remove the device at bedtime and before showering, swimming and other water activities. After the wearing period the devices were returned to NHANES by mail, where the data were downloaded and the devices checked for calibration. The accelerometer monitors the intensity of vertical body movements in a one-minute time interval and records these intensities as "counts per minute" which were used to classify activity period in different intensity. Non-wear was defined as a period of $>60$ min with 0 counts, with allowance of 2 min of counts between 0 and 100. For our analysis, time spend in sedentary activity ( $<100$ counts per minute) and moderate to vigorous physical activity (MVPA) (>2020 counts per minute) were calculated. ${ }^{17}$ The proportion of time spent sedentary and time spent in MVPA was calculated by dividing the total time spent in each activity by total wear time. Hence, 
percent sedentary time and percent MVPA are to be interpreted as the average proportion of one day spent in this activity.

\section{Cardiorespiratory Fitness}

Participants performed a submaximal treadmill test in order to estimate maximal oxygen uptake $\left(\mathrm{VO}_{2 \max }\right)$ as a measure for cardiorespiratory fitness; based on sex, age, $\mathrm{BMI}$, and self-reported level of PA one of the eight different intensity protocols was chosen. Participants at increased risk for complications from exercise or with conditions that might affect the $\mathrm{VO}_{2 \max }$ test, were excluded. The objective of every protocol was to provoke a heart rate that was approximately $75 \%$ of the age-predicted maximum by the end of the test. The exercise test included a 2-min warm-up period, two 3-minute submaximal exercise stages and a 2-min cool down period, but varied in grade and speed depending on protocol. Heart rate was monitored continuously using four electrodes connected to thorax and abdomen. The heart rate at the end of each stage was used to estimate $\mathrm{VO}_{2 \max }$. A detailed description of test procedures can be found in the NHANES Cardiovascular Fitness Procedure Manual. ${ }^{18}$

\section{Cardio-Metabolic Outcomes}

WC was measured over the iliac crest. BMI $\left(\mathrm{kg} / \mathrm{m}^{2}\right)$ was calculated from height and body weight, which were measured with a fixed stadiometer and a Toledo digital floor scale respectively. SBP and DBP ( $\mathrm{mmHg}$ ) were measured up to four times on the upper right arm and were reported as the mean of all readings except the first, if three or four measurements were available. If participants had two valid blood pressure readings, only the last reading was used in the analysis. If participants had only one reading, that reading was used. Fasting blood samples were collected in the selected population. Glucose values were determined using the hexokinase method, with the Cobas Mira analyzer (Roche Diagnostic Systems, Inc., Montclair, NJ, USA). Serum concentrations of CRP were measured with latex-enhanced nephelometry using a Dade Behring Nephelometer II Analyzer System (Dade Behring Diagnostics, Inc., Newark, DE, USA). Serum levels total Cholesterol, HDL-Cholesterol and TG were determined with a Hitachi 704 Analyzer (Roche Diagnostics (formerly Boehringer-Mannheim Diagnostics), Indianapolis, IN, USA). Metabolic syndrome was defined as meeting three or more of the following criteria: (1) WC $\geq 102 \mathrm{~cm}$ for men and $\geq 88 \mathrm{~cm}$ for women; (2) serum triglyceride level of $\geq 1.7 \mathrm{mmol} / \mathrm{L}$; (3) HDL cholesterol level $\leq 1.03 \mathrm{mmol} / \mathrm{L}$ for men and $\leq 1.30$ $\mathrm{mmol} / \mathrm{L}$ for women; (4) fasting glucose level $\geq 5.6 \mathrm{mmol} / \mathrm{L}$ or use of antidiabetic medications (insulin or oral agents); or (5) systolic blood pressure $\geq 130 \mathrm{mmHg}$ and/or diastolic blood pressure $\geq 85 \mathrm{mmHg}$, or use of antihypertensive medications. ${ }^{19}$ 


\section{Covariates}

Age (at screening time), sex, self-reported ethnicity (non-Hispanic white, non-Hispanic black, Mexican American and other), self-reported health status (poor to excellent on 5point scale) and smoking status were included in the analyses as covariates. Participants with serum cotinine levels $>10 \mathrm{ng} / \mathrm{mL}$ were considered current smokers. ${ }^{20}$

\section{Statistical Analysis}

To obtain population representative findings, all analyses took into account the NHANES complex survey design including sample weights, stratification and clustering. The NHANES 2003-2004 fasting sample weights were used. Complex samples analyses were performed using IBM SPSS Statistics for Windows, Version 20.0. (IBM Corp., Armonk, NY, USA). Significance level was set at $<0.05$. Because of non-normal distribution, log transformations were used for TG, and CRP. Descriptive statistics included means and standard errors for continuous variables (age, WC, BMI, accelerometry data, fitness, blood pressure and blood sample values). Data are presented for total sample and for men and women separately to illustrate differences in the main characteristics.

In further analyses data from men and women were combined. The interactions between sex and sedentary time, sex and MVPA and sex and fitness with the cardiometabolic outcome measures were tested in all final models, all tested interactions were not statistically significant. Cardio-metabolic outcomes are presented as means with standard errors according to sex specific quartiles of the three main predictors (percent sedentary time, percent MVPA time and fitness). Differences in cardio-metabolic outcomes between quartiles of sedentary time, MVPA and fitness were tested with ANOVA.

Multiple linear regression analyses were performed with the cardio-metabolic markers as dependent variables and percent sedentary time, percent MVPA time and fitness as the independent variables. Model 1 was adjusted for age, sex, ethnicity, smoking status and self-rated health status. BMI was added in Model 2. Additionally, MVPA and fitness were added in the model of sedentary time; sedentary time and fitness were added in the model of MVPA; and sedentary time and MVPA were added in the model of fitness. Standardized regression coefficients are presented to allow for determining the relative importance of the independent variables. ${ }^{21}$ Logistic regression analyses were performed to assess the association between quartiles of sedentary time, MVPA, and fitness with metabolic syndrome. Analyses were adjusted for age, sex, ethnicity, smoking status and self-rated health status. Additionally, MVPA and fitness were added in the model of sedentary time; sedentary time and fitness were added in the model of MVPA; and sedentary time and MVPA were added in the model of fitness. 


\section{Results}

Participant characteristics are shown in Table 4.1. The age range of the 543 participants, of whom 55\% were men, was 18-49 years, with an average of 32 years and the mean BMI was $27.1 \mathrm{~kg} / \mathrm{m}^{2}$. The mean values for the blood samples were all within the normal range. Average percentage of sedentary time was 53\% and average percentage of time spent in MVPA was 4.1\%. Fitness (predicted $\mathrm{VO}_{2 \max }$ ) was 42.6 and 35.7 $\mathrm{mL} / \mathrm{kg} / \mathrm{min}$ for men and women, respectively. The correlation between sedentary time and MVPA was 0.38 and between sedentary and fitness was 0.11 . A correlation of 0.29 was found between MVPA and fitness.

Table 4.1. Participant demographics, accelerometry measures, fitness and cardiometabolic markers.

\begin{tabular}{|c|c|c|c|c|c|c|}
\hline \multirow{2}{*}{$\begin{array}{l}\text { Characteristic } \\
\text { Age (years), mean } \pm \text { SE }\end{array}$} & \multicolumn{2}{|c|}{$\begin{array}{c}\text { Total } \\
(N=543)\end{array}$} & \multicolumn{2}{|c|}{$\begin{array}{c}\text { Men } \\
(N=297)\end{array}$} & \multicolumn{2}{|c|}{$\begin{array}{l}\text { Women } \\
(N=246)\end{array}$} \\
\hline & 32.19 & 0.57 & 32.02 & 0.91 & 32.37 & 0.73 \\
\hline Ethnicity, \% & & & & & & \\
\hline non-Hispanic white & 70.35 & & 69.18 & & 71.61 & \\
\hline non-Hispanic black & 11.04 & & 11.13 & & 10.95 & \\
\hline Mexican American & $11.1 \%$ & & 12.72 & & 9.46 & \\
\hline Other & 7.45 & & 6.97 & & 7.98 & \\
\hline Waist Circumference $(\mathrm{cm})$, mean \pm SE & 92.74 & 0.63 & 96.74 & 0.86 & 88.40 & 0.62 \\
\hline Body Mass Index $\left(\mathrm{kg} / \mathrm{m}^{2}\right)$, mean $\pm \mathrm{SE}$ & 27.05 & 0.25 & 27.56 & 0.28 & 26.51 & 0.35 \\
\hline Average valid wear time (min), mean $\pm \mathrm{SE}$ & 851.87 & 4.50 & 861.68 & 5.82 & 841.21 & 8.62 \\
\hline Valid days, $\%$ & & & & & & \\
\hline $\mathrm{N}$ valid days $=1$ & 6.81 & & 5.72 & & 8.13 & \\
\hline $\mathrm{N}$ valid days $=2$ & 7.73 & & 8.42 & & 6.91 & \\
\hline$N$ valid days $\geq 3$ & 85.46 & & 85.86 & & 84.96 & \\
\hline Percent Sedentary Time $(\%)$, mean \pm SE & 53.20 & 0.50 & 51.44 & 0.78 & 55.11 & 0.66 \\
\hline Sedentary Time per day (min), mean \pm SE & 455.03 & 4.90 & 444.61 & 5.52 & 466.36 & 7.19 \\
\hline Percent MVPA a (\%), mean \pm SE & 4.13 & 0.17 & 5.11 & 0.23 & 3.06 & 0.14 \\
\hline MVPA a during day (min), mean \pm SE & 35.07 & 1.40 & 43.82 & 1.96 & 25.55 & 1.30 \\
\hline Fitness $(\mathrm{mL} / \mathrm{kg} / \mathrm{min})$, mean $\pm \mathrm{SE}$ & 39.31 & 0.49 & 42.62 & 0.43 & 35.71 & 0.70 \\
\hline $\mathrm{SBP}$ b $(\mathrm{mmHg})$, mean $\pm \mathrm{SE}$ & 113.66 & 0.41 & 117.18 & 1.02 & 109.83 & 0.60 \\
\hline $\mathrm{DBP}^{\mathrm{b}}(\mathrm{mmHg})$, mean $\pm \mathrm{SE}$ & 69.27 & 0.56 & 70.65 & 0.68 & 67.76 & 0.79 \\
\hline Fasting Glucose $(\mathrm{mmoL} / \mathrm{L})$, mean $\pm \mathrm{SE}$ & 5.11 & 0.03 & 5.25 & 0.04 & 4.96 & 0.04 \\
\hline HDL-cholesterol $(\mathrm{mmoL} / \mathrm{L})$, mean $\pm \mathrm{SE}$ & 1.42 & 0.02 & 1.28 & 0.04 & 1.56 & 0.04 \\
\hline non-HDL-Cholesterol $(\mathrm{mmoL} / \mathrm{L})$, mean $\pm \mathrm{SE}$ & 3.48 & 0.04 & 3.60 & 0.08 & 3.34 & 0.05 \\
\hline Triglycerides $(\mathrm{mmoL} / \mathrm{L}) \mathrm{c}$, mean $\pm \mathrm{SE}$ & 1.19 & 0.04 & 1.31 & 0.05 & 1.07 & 0.05 \\
\hline C-Reactive Protein $(\mathrm{mg} / \mathrm{L})^{c}$, mean $\pm \mathrm{SE}$ & 1.44 & 0.09 & 1.16 & 0.11 & 1.82 & 0.11 \\
\hline Metabolic syndrome, $\%$ & 16.77 & & 19.40 & & 13.91 & \\
\hline
\end{tabular}

a MVPA: Moderate to vigorous physical activity; b SBP, DBP: Systolic and diastolic blood pressure; c back transformed from log scale 
In Table 4.2 the cardio-metabolic outcomes are presented by sex-specific quartiles of sedentary time, MVPA, and fitness. Cardio-metabolic outcomes did not differ significantly between quartiles of sedentary time (all p-values > 0.05). More time spent in MVPA was associated with a healthier cardio-metabolic risk profile. For example, BMI was $28.5 \mathrm{~kg} / \mathrm{m} 2$ in the lowest MVPA quartile (least active) compared with $24.9 \mathrm{~kg} / \mathrm{m} 2$ in the highest quartile (most active). Between quartile differences were significant for all outcome measures (all $p<0.05)$, except for SBP $(p=0.13)$ and glucose $(p=0.09)$. Furthermore higher fitness was linked with advantageous values for most of the car dio-metabolic risk factors. However, differences in SBP, HDL-cholesterol, fasting glucose and TG were not significant $(p \geq 0.05)$.

To establish the relative importance of sedentary time, MVPA and fitness as predictors for the cardio-metabolic markers standardized regression coefficients are presented in Table 4.3. Sedentary time was only associated with levels of HDL-cholesterol $(\beta=$ $-0.080, p=0.05)$ and TG $(\beta=0.080, p=0.03)$ (Model 2$)$. These results became nonsignificant after adjustment for MVPA and fitness. MVPA was negatively associated with WC $(\beta=-0.226, p=0.01)$, BMI $(\beta=-0.239$, $p<0.01)$ (Model 1), and TG $(\beta=-0.108$, $\mathrm{p}=0.01$, Model 2$)$ and positively associated with HDL cholesterol $(\beta=0.144, \mathrm{p}<0.01)$ (Model 2). Significant associations for BMI, WC, HDL-cholesterol and TG remained after additional adjustment for sedentary time and fitness. Fitness was significantly associated with WC $(\beta=-0.287, \mathrm{p}<0.01)$, BMI $(\beta=-0.266, \mathrm{p}<0.01)$ (Model 1), SBP $(\beta=$ -0.159, $\mathrm{p}=0.03)$, TG $(\beta=-0.092, \mathrm{p}<0.01)$, and CRP $(\beta=-0.130, \mathrm{p}<0.01)$ (Model 2). These associations remained significant after adjustment for sedentary time and MVPA.

The strongest independent associations of MVPA and fitness were found with WC and BMI (Table 4.3). For these outcomes, the adjusted mean difference in WC and BMI between the most active and the least active and between the least fit and most fit were calculated. Results reveal a $5.6 \mathrm{~cm}$ difference in WC and $2.4 \mathrm{~kg} / \mathrm{m}^{2}$ difference in BMI between the least active (lowest quartile of MVPA) and most active (highest quartile of MVPA). Similarly, a $6.3 \mathrm{~cm}$ difference in WC and a $2.3 \mathrm{~kg} / \mathrm{m} 2$ difference in BMI was found between the least fit and the most fit individuals (not tabulated).

The associations between sedentary time, MPVA, and fitness with metabolic syndrome were similar to the results of the individual cardio-metabolic risk factors (not tabulated). Sedentary time was not associated with metabolic syndrome while MVPA and fitness were significantly associated with the presence of metabolic syndrome. Compared with the highest quartile of MVPA (most active); those with lower levels of MVPA had a significantly higher likelihood of metabolic syndrome in the fully adjusted model (quartile 3: odds ratio $(\mathrm{OR})=4.24$ (95\% confidence interval (CI): 1.49; 2.06); 
quartile 2: $\mathrm{OR}=4.43$ (95\%CI: 1.06; 18.61); quartile 1: OR = 7.96 (95\%CI: 1.91; 33.09). Similarly, compared to the most fit, a lower fitness was associated with an increased likelihood of metabolic syndrome (quartile 3: OR = 1.14 (95\%CI: 0.42; 3.08); quartile 2: $\mathrm{OR}=1.69$ (95\%CI: 0.59; 4.82); quartile $1: \mathrm{OR}=2.08$ (95\%CI: 1.12; 3.87). In additional analyses we selected participants with three or more valid; results remained similar (data not shown).

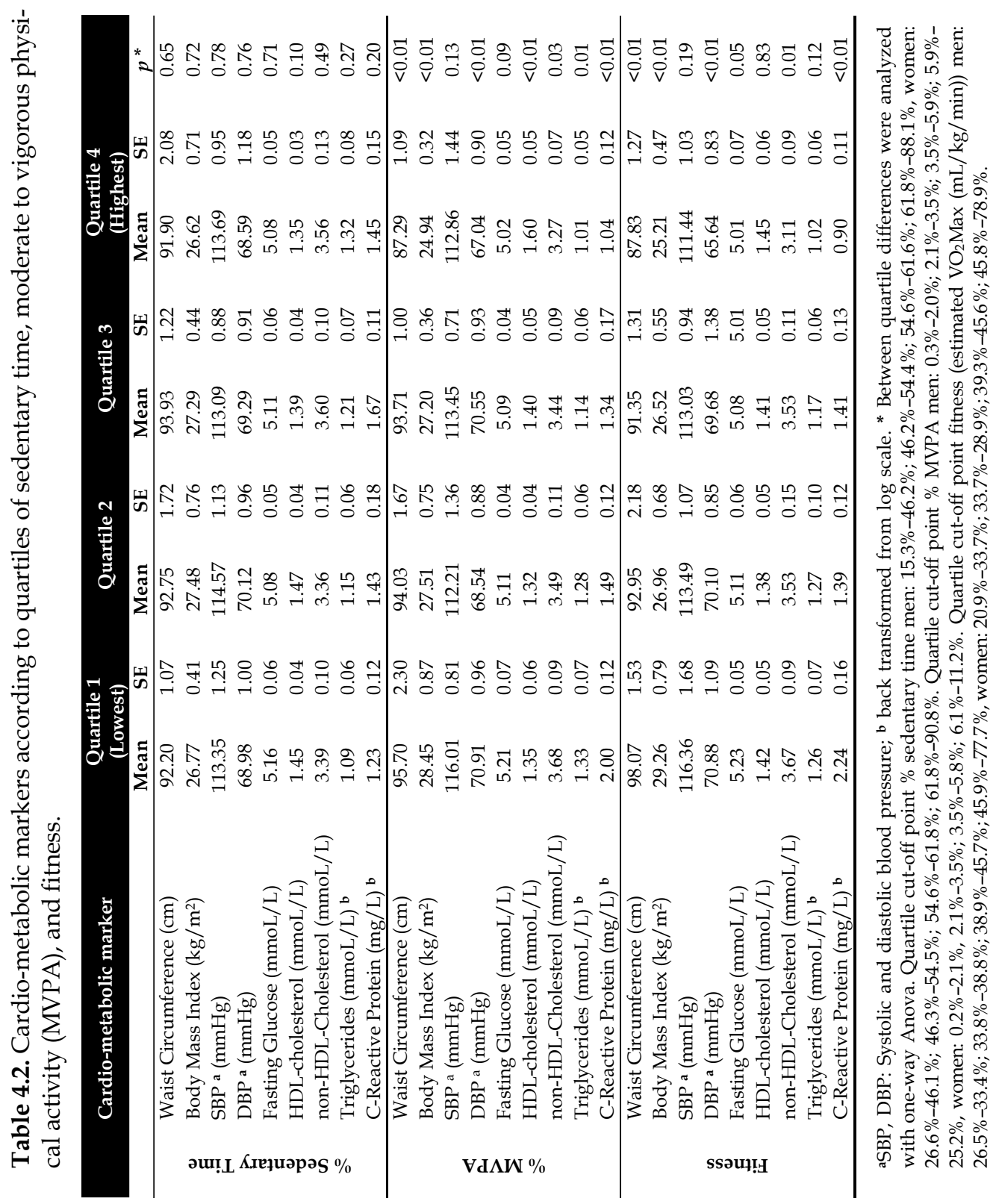




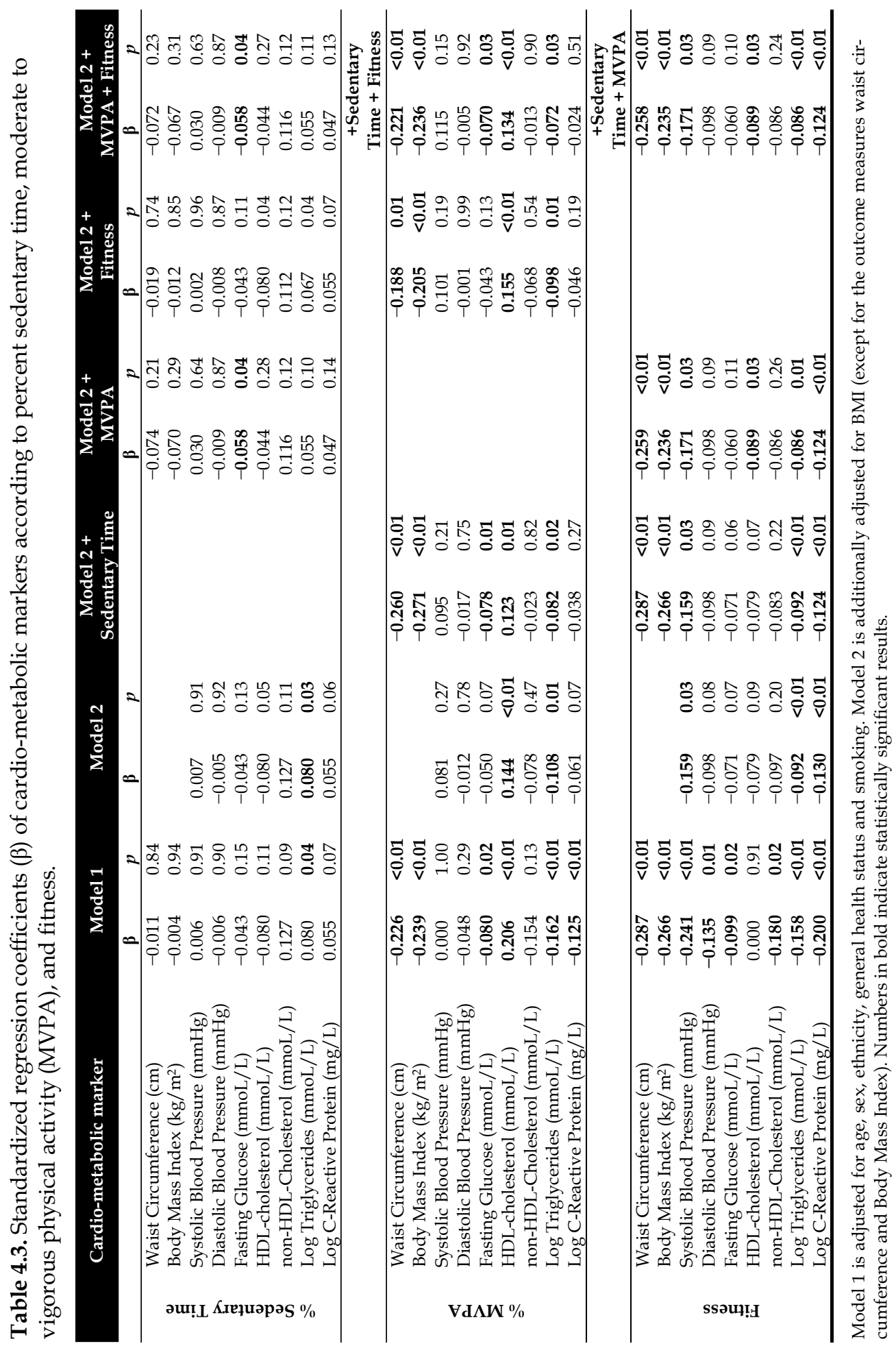




\section{Discussion}

Using data from NHANES 2003-2004, this study is one of the first studies to evaluate the independent associations between objectively measured sedentary time, MVPA and fitness with a wide range of cardio-metabolic risk factors in a sample of US adults aged 18-49 years. Higher sedentary time was associated with lower levels of HDL cholesterol and higher levels TG but these associations were, however, not independent of MVPA and fitness. In contrast, both MVPA and fitness had an independent beneficial effect on cardio-metabolic risk. A higher level of MVPA was associated with lower WC, BMI, fasting glucose and a more favourable lipid profile (lower TG and higher HDL cholesterol), independent of sedentary time and fitness. Higher fitness was associated with lower WC, BMI and TG levels, and in addition with lower SBP, HDL and lower levels of the inflammatory marker CRP, independent of sedentary time and MVPA. Additionally, lower levels of MVPA and fitness were independently associated with a greater likelihood of metabolic syndrome.

Previous studies have shown that higher self-reported sedentary time is associated with several negative health outcomes 22,23 and more sedentary time was also associated with poor metabolic health and mortality when objective measures were used. ${ }^{24-26} \mathrm{In}$ addition, several authors examined the relationship between sedentary time and metabolic health using data from NHANES. ${ }^{-8} 8$ Bankoski et al. ${ }^{6}$ and Genusso et al. ${ }^{8}$ demonstrated in an older subpopulation ( $\geq 60$ and $\geq 65$ years respectively) that more sedentary time was associated with an increased metabolic risk independent of MVPA. Another study in a subpopulation of $\geq 20$ years showed a linear association between sedentary time and several cardio-metabolic health outcomes, independent of MVPA. ${ }^{7}$ However, in accordance with our results, not all studies have shown an association between higher sedentary time and poor health independent of MVPA. For example, sedentary time and metabolic risk were not associated in subjects with a mean age of 41 years, ${ }^{27}$ or in another study in adolescents after correcting for MVPA. ${ }^{28}$ Moreover, all studies mentioned above did not include fitness in their analyses.

The lack of an independent association between sedentary time and cardio-metabolic risk in the current analyses of the NHANES data study seems in contradiction with the conclusions of Healy et al. ${ }^{7}$, as these authors also used data from the same NHANES survey. A different approach in statistical analysis might explain this, as Healy et al. performed a trend analysis. Differences in the age of the subjects might also explain these differences (mean age 32.3 years in the present analyses vs. 46.5 years in the analyses by Healy et al.).

Sedentary time may not be a risk factor for poor cardio-metabolic health in younger adults, as the effects of sedentary time possibly only become apparent in an aging pop- 
ulation or when levels of MVPA and fitness decline below a certain threshold. More research is needed to determine the relative importance of sedentary behaviour as age increases and energy expenditure in other activities decline.

In our study, more MVPA and higher fitness were associated with several markers of better cardio-metabolic health and with lower likelihood of metabolic syndrome. Also earlier studies observed positive effects of MVPA and fitness on (cardio-metabolic) health. ${ }^{29-31}$ In line with our results, both higher levels of MVPA and fitness were independently associated with lower cardio-metabolic risk, ${ }^{32-34}$ and inflammatory markers, ${ }^{34}$ when the effect of the other was taken into account. Other authors have compared the effects of MVPA and fitness on cardio-metabolic outcomes, and fitness seems more strongly related to cardio-metabolic outcomes and mortality than MVPA.11,14,35 However, these results were limited by the use of self-reported PA levels and these studies did not include sedentary time in their analyses. In this study, MVPA was associated with better cardio-metabolic health independent of fitness suggesting that unfit individuals could benefit from MVPA in terms of increasing metabolic health. Furthermore, fitness was associated with better cardio-metabolic health independent of MVPA, suggesting MVPA and fitness are two distinct determinants of cardio-metabolic health.

PA and fitness are related, as fitness increases with increased MVPA; other determinants of fitness include age, sex, body composition and genetic factors. ${ }^{36}$ Fitness is considered to be a more stable trait than MVPA and decreases slowly when a person does not engage in MVPA. This could be an explanation that the observed associations of PA and fitness with metabolic health in our study do not show significant associations with the same cardio-metabolic markers. Because PA and fitness are related it could be suggested that fitness modifies the associations between PA and health. Blair et al. ${ }^{37}$ and Franks et al. ${ }^{38}$ have demonstrated that fitness modified the association between PA and metabolic syndrome. Especially people in the low fitness group would benefit from an increase in PA. Ekblom-Bak et al., ${ }^{32}$ found that CVD risk clustered around people with low PA and low fitness. These studies all used self-reported measures of PA. In future studies, the modifying effect of fitness should preferably be studied with objective measures for PA and fitness. Because of the limited sample size in our study, we could not determine the combined effects of PA and fitness.

A strength of our study is the use of objective measures for sedentary time, MVPA and fitness, where previous studies have predominately used self-reported measures for PA. Self-reported measures of PA show limited validity and reliability. ${ }^{39}$ The use of a sub-maximal exercise test to measure fitness is not optimal, but the estimates from submaximal exercise tests correlate strongly with the estimates from maximal exercise tests. ${ }^{40}$ This report also has some limitations. Fitness was estimated using eight different 
protocols, intended to reach $75 \%$ of a participant's estimated maximum heart rate by the end of stage 2. However, not all participants, especially those who had a high BMI, reached their target heart rate. This may have influenced the validity of the estimated $\mathrm{VO}_{2}$ max. Accelerometers such as the ActiGraph used in this study, are most accurate in measuring ambulatory activities. Activities such as cycling are poorly detected and the device was not worn during water activities such as swimming. The cut-points used to define sedentary time and MVPA have been validated in younger adults and have been widely used, also in previous NHANES articles, ${ }^{17,41}$ however, they may not be appropriate for specific subgroups, for example all age-groups. Further, we did not have information about the type of physical activity as the use of accelerometry only gives us a measure of overall physical activity. A recent study shows that leisure time PA is beneficially associated with cardiovascular health, whereas occupational PA has been associated with detrimental health. ${ }^{42}$ Because of the cross sectional design it is not possible to determine causality and longitudinal studies are needed to investigate the causal relationship of sedentary time, MVPA and fitness on metabolic health. Our analyses were adjusted for several confounders, however, residual confounding, for instance by dietary factors, may remain. Further, we did not adjust for multiple testing, consequently some of our significant findings may have emerged from type 1 errors. However, results for metabolic syndrome as an outcome were similar to the individual cardio-metabolic risk factors. Finally, NHANES is designed to provide a representative sample of the US population. However due to exclusion criteria applied during the fitness test, our study population probably does not reflect the overall population. The current analyses are based on a population without a history of, or symptoms of cardiovascular diseases, severe asthma, or other self-reported physical conditions preventing them to perform the fitness test, reducing the generalizability of our results. More research, in different populations using objective measures for sedentary time, PA and fitness is needed to determine the independent and combined effects of PA and fitness with health outcomes.

\section{Conclusion}

Based on our results we suggest that a combination of increasing MVPA and fitness is most beneficial to decrease cardio-metabolic risk in this adult US population. Sedentary time appears to be of minor importance for cardio-metabolic risk in this population. In the light of public health these results suggest that a relative young and healthy adult population would benefit less from interventions only directed to reduce sitting time, in terms of cardio-metabolic risk. 


\section{References}

1. Li J, Siegrist J. Physical activity and risk of cardiovascular disease-a meta-analysis of prospective cohort studies. Int. J. Environ. Res. Public. Health 2012;9(2):391-407.

2. Warburton DE, Nicol CW, Bredin SS. Health benefits of physical activity: the evidence. CMAJ 2006;174(6):801-9.

3. Physical Activity Guidelines Advisory Committee Report, 2008. Available online: http://www. health.gov/paguidelines/Report/pdf/CommitteeReport.pdf (accesed on 20 november 2014).

4. Hamilton MT, Healy GN, Dunstan DW, Zderic TW, Owen N. Too little exercise and too much sitting: inactivity physiology and the need for new recommendations on sedentary behavior. Curr Cardiovasc Risk Rep 2008;2(4):292-298.

5. Pate RR, O'Neill JR, Lobelo F. The evolving definition of" sedentary". Exerc. Sport Sci. Rev. 2008;36(4):173-178.

6. Bankoski A, Harris TB, McClain JJ, Brychta RJ, Caserotti P, Chen KY, Berrigan D, Troiano RP, Koster A. Sedentary activity associated with metabolic syndrome independent of physical activity. Diabetes Care 2011;34(2):497-503.

7. Healy GN, Matthews CE, Dunstan DW, Winkler EA, Owen N. Sedentary time and cardiometabolic biomarkers in US adults: NHANES 2003-06. Eur. Heart J. 2011;32(5):590-7.

8. Gennuso KP, Gangnon RE, Matthews CE, Thraen-Borowski KM, Colbert LH. Sedentary behavior, physical activity, and markers of health in older adults. Med. Sci. Sports Exerc. 2013;45(8):1493-1500.

9. Owen N, Healy GN, Matthews CE, Dunstan DW. Too much sitting: the population health science of sedentary behavior. Exerc. Sport Sci. Rev. 2010;38(3):105-13.

10. LaMonte MJ, Barlow CE, Jurca R, Kampert JB, Church TS, Blair SN. Cardiorespiratory fitness is inversely associated with the incidence of metabolic syndrome a prospective study of men and women. Circulation 2005;112(4):505-512.

11. Lee D, Sui X, Ortega F, Kim Y, Church T, Winett R, Ekelund U, Katzmarzyk P, Blair S. Comparisons of leisure-time physical activity and cardiorespiratory fitness as predictors of allcause mortality in men and women. Br. J. Sports Med. 2011;45(6):504-10.

12. Lee D-c, Sui X, Artero EG, Lee I-M, Church TS, McAuley PA, Stanford FC, Kohl HW, Blair SN. Long-term effects of changes in cardiorespiratory fitness and body mass index on all-cause and cardiovascular disease mortality in men the Aerobics Center Longitudinal Study. Circulation 2011;124(23):2483-2490.

13. Minder CM, Shaya GE, Michos ED, Keenan TE, Blumenthal RS, Nasir K, Carvalho JA, Conceição $\mathrm{RD}$, Santos RD, Blaha MJ. Relation between self-reported physical activity level, fitness, and cardiometabolic risk. Am. J. Cardiol. 2014;113(4):637-643.

14. Sassen B, Cornelissen VA, Kiers H, Wittink H, Kok G, Vanhees L. Physical fitness matters more than physical activity in controlling cardiovascular disease risk factors. Eur. J. Cardiovasc. Prev. Rehabil. 2009;16(6):677-683.

15. Williams PT. Physical fitness and activity as separate heart disease risk factors: a meta-analysis. Med. Sci. Sports Exerc. 2001;33(5):754.

16. National health and nutrition examination survey data NHANES 2003-2004. Available online: Http://wwwn.Cdc.Gov/nchs/nhanes/search/nhanes03_04.Aspx (accesed on 05 January 2017).

17. Troiano RP, Berrigan D, Dodd KW, Masse LC, Tilert T, McDowell M. Physical activity in the United States measured by accelerometer. Med. Sci. Sports Exerc. 2008;40(1):181.

18. National health and nutrition examination survey: Cardiovascular fitness procedures manual. Available online: http://www.Cdc.Gov/nchs/nhanes/nhanes20032004/cvx_c.Htm\#protocol_and _procedure (accesed on 20 november 2014).

19. Grundy SM, Cleeman JI, Daniels SR, Donato KA, Eckel RH, Franklin BA, Gordon DJ, Krauss RM, Savage PJ, Smith SC. Diagnosis and management of the metabolic syndrome an American Heart Association/National Heart, Lung, and Blood Institute scientific statement. Circulation 2005;112(17):2735-2752.

20. Nelson RK, Horowitz JF, Holleman RG, Swartz AM, Strath SJ, Kriska AM, Richardson CR. Daily physical activity predicts degree of insulin resistance: a cross-sectional observational study using the 2003-2004 National Health and Nutrition Examination Survey. Int. J. Behav. Nutr. Phys. Act. 2013;10(10):10.1186. 
21. Bring J. How to standardize regression coefficients. Am. Stat. 1994;48(3):209-213.

22. Ford ES, Caspersen CJ. Sedentary behaviour and cardiovascular disease: a review of prospective studies. Int. J. Epidemiol. 2012;41(5):1338-53.

23. Thorp AA, Owen N, Neuhaus M, Dunstan DW. Sedentary behaviors and subsequent health outcomes in adults: a systematic review of longitudinal studies, 1996-2011. Am. J. Prev. Med. 2011;41(2):207-215.

24. Healy GN, Wijndaele K, Dunstan DW, Shaw JE, Salmon J, Zimmet PZ, Owen N. Objectively measured sedentary time, physical activity, and metabolic risk the Australian Diabetes, Obesity and Lifestyle Study (AusDiab). Diabetes Care 2008;31(2):369-371.

25. Helmerhorst HJ, Wijndaele K, Brage S, Wareham NJ, Ekelund U. Objectively measured sedentary time may predict insulin resistance independent of moderate-and vigorous-intensity physical activity. Diabetes 2009;58(8):1776-1779.

26. Koster A, Caserotti P, Patel KV, Matthews CE, Berrigan D, Van Domelen DR, Brychta RJ, Chen KY, Harris TB. Association of sedentary time with mortality independent of moderate to vigorous physical activity. PLoS ONE 2012;7(6):e37696.

27. Scheers $T$, Philippaerts R, Lefevre J. SenseWear-determined physical activity and sedentary behavior and metabolic syndrome. Med. Sci. Sports Exerc. 2013;45(3):481-489.

28. Ekelund U, Luan Ja, Sherar LB, al. e. Moderate to vigorous physical activity and sedentary time and cardiometabolic risk factors in children and adolescents. JAMA 2012;307(7):704-712.

29. Blair SN, Kampert JB, Kohl HW, Barlow CE, Macera CA, Paffenbarger RS, Gibbons LW. Influences of cardiorespiratory fitness and other precursors on cardiovascular disease and all-cause mortality in men and women. JAMA 1996;276(3):205-210.

30. Haskell WL, Lee I-M, Pate RR, Powell KE, Blair SN, Franklin BA, Macera CA, Heath GW, Thompson PD, Bauman A. Physical activity and public health: updated recommendation for adults from the American College of Sports Medicine and the American Heart Association. Circulation 2007;116(9):1081.

31. Warburton DE, Nicol CW, Bredin SS. Health benefits of physical activity: the evidence. Can. Med. Assoc. J. 2006;174(6):801-809.

32. Ekblom-Bak E, Hellenius M-L, Ekblom Ö, Engström L-M, Ekblom B. Independent associations of physical activity and cardiovascular fitness with cardiovascular risk in adults. Eur. J. Cardiovasc. Prev. Rehabil. 2010;17(2):175-180.

33. O'Donovan G, Hillsdon M, Ukoumunne OC, Stamatakis E, Hamer M. Objectively measured physical activity, cardiorespiratory fitness and cardiometabolic risk factors in the Health Survey for England. Prev. Med. 2013;57(3):201-205.

34. Lavie CJ, Church TS, Milani RV, Earnest CP. Impact of physical activity, cardiorespiratory fitness, and exercise training on markers of inflammation. JCRP 2011;31(3):137-145.

35. Myers J, Kaykha A, George S, Abella J, Zaheer N, Lear S, Yamazaki T, Froelicher V. Fitness versus physical activity patterns in predicting mortality in men. Am. J. Med. 2004;117(12):912-8.

36. Bouchard C, Rankinen T. Individual differences in response to regular physical activity. Med. Sci. Sports Exerc. 2001;33(6 Suppl):S446-51; discussion S452-3.

37. Blair SN, Cheng Y, Holder JS. Is physical activity or physical fitness more important in defining health benefits? Med. Sci. Sports Exerc. 2001;33(6; SUPP):S379-S399.

38. Franks PW, Ekelund U, Brage S, Wong M-Y, Wareham NJ. Does the association of habitual physical activity with the metabolic syndrome differ by level of cardiorespiratory fitness? Diabetes Care 2004;27(5):1187-1193.

39. Shephard RJ. Limits to the measurement of habitual physical activity by questionnaires. Br. J. Sports Med. 2003;37(3):197-206.

40. Faulkner J, Parfitt G, Eston R. Prediction of maximal oxygen uptake from the ratings of perceived exertion and heart rate during a perceptually-regulated sub-maximal exercise test in active and sedentary participants. Eur. J. Appl. Physiol. 2007;101(3):397-407.

41. Tudor-Locke C, Camhi SM, Troiano RP. A catalog of rules, variables, and definitions applied to accelerometer data in the National Health and Nutrition Examination Survey, 2003-2006. Prev. Chronic. Dis. 2012;9:110332.

42. Li J, Loerbroks A, Angerer P. Physical activity and risk of cardiovascular disease: what does the new epidemiological evidence show? Curr. Opin. Cardiol. 2013;28(5):575-583. 



\section{Chapter 5}

Sedentary time and higher intensity physical activity versus cardio respiratory fitness; what is more important for cardio-metabolic health for adults aged 40-75?

- The Maastricht Study -

Jeroen HPM van der Velde, Nicolaas C Schaper, Coen DA Stehouwer, Carla JH van der Kallen, Simone JS Sep, Miranda T Schram, Ronald MA Henry, Pieter C Dagnelie, Simone JPM Eussen, Martien CJM van Dongen, Hans HCM Savelberg, Annemarie Koster 


\section{Abstract}

Aim: Examine the independent and combined associations of sedentary time (ST), higher intensity physical activity (HPA), and cardiorespiratory fitness (CRF) with the metabolic syndrome (MetS), and diabetes status.

Research Design and Methods: In 1,933 adults (aged 40-75 years) ST and HPA were measured with the activPAL3. CRF was assessed by sub-maximal cycle-ergometer testing. MetS was defined according to the ATP III guidelines. Diabetes status (normal, prediabetes, type 2 diabetes (T2DM)) was determined from oral glucose tolerance test. (Multinomial) logistic regression analyses were used to calculate likelihood for MetS, prediabetes and T2DM for ST, HPA, and CRF separately and for combinations of STCRF and HPA-CRF.

Results: Higher ST, lower HPA and lower CRF were independently of each other associated with greater odds for MetS and T2DM. Compared with $\mathrm{CRF}_{\text {high }}-\mathrm{HPA}_{\text {high, }}$ odds for MetS and T2DM were higher in groups with a lower CRF regardless of HPA. $\mathrm{CRF}_{\text {low }}-\mathrm{HPA}_{\text {low }}$ had a particularly high odds for MetS (OR=5.73, 95\%CI:(3.84,8.56)) and T2DM $(\mathrm{OR}=6.42(3.95,10.45))$. Similarly, compared to $\mathrm{CRF}_{\text {high }}-\mathrm{ST}_{\text {low }}$, those with medium or low CRF had higher odds for MetS, prediabetes and T2DM, irrespective of ST. In those with high CRF, high ST was associated with significantly high odds for MetS $(\mathrm{OR}=2.93(1.72,4.99))$ and T2DM $(\mathrm{OR}=2.21(1.17,4.17))$. Highest odds of MetS, prediabetes and $\mathrm{T} 2 \mathrm{DM}$ was observed in $\mathrm{CRF}_{\text {low }}-\mathrm{ST}_{\text {high; }} \mathrm{OR}_{\text {Mets }}=9.22(5.74,14.80), \quad \mathrm{OR}_{\text {prediabe- }}$ tes $=3.44(1.89,6.26),\left(\mathrm{OR}_{\mathrm{T} 2 \mathrm{DM}} 8.38(4.83,14.55)\right.$.

Conclusion: These data suggest that all three components, i.e. ST, HPA, and CRF, should be addressed in order to optimally improve cardio-metabolic health 


\section{Introduction}

Globally, cardiovascular disease (CVD) is the number one cause of death and contributes substantially to the accelerating costs of health care. ${ }^{1}$ Moderate to vigorous intensity physical activity (MVPA) is a key non-pharmacological strategy to reduce the risk for CVD. ${ }^{2}$ However, only a small part of daily activities comprises of MVPA. Generally, the majority of the day is spent in sedentary behavior. ${ }^{3}$ Sedentary behavior refers to any waking behavior characterized by an energy expenditure $\leq 1.5$ METs while in a sitting or reclining position. ${ }^{4}$ Emerging evidence indicates that the amount of sedentary time (ST) is a determinant of cardio-metabolic health.5,6 Although these effects are probably independent of MVPA, the interrelationships between sedentary behavior, MVPA and cardio-metabolic health need further clarification, as recently concluded by the American Heart Association. ${ }^{7}$

When examining the relationships between sedentary behavior and MVPA with cardio-metabolic health, cardiorespiratory fitness (CRF) should also be taken into account. CRF (or aerobic capacity) has been considered as an important determinant of cardiometabolic health in many studies. ${ }^{8,9}$ Differences in CRF between individuals are partly explained by differences in frequency and intensity of engagement in PA. An estimated $10-50 \%$ of CRF is explained by other factors than PA, including genetic differences, behavioral, and environmental elements. ${ }^{10}$ Recent studies have shown a inverse association between ST and CRF.11-13 Consequently, it could be possible to engage regularly in MVPA and not have a high CRF, or to have a high CRF without frequently engaging in MVPA. ${ }^{14}$

Although to some extent interrelated, MVPA, ST and CRF should be considered different traits and may be independently associated with cardio-metabolic health. ${ }^{14}$ In addition, these traits occur in combination of each other, but which combination is most optimal or most harmful? Some studies reported that the greatest cardio-metabolic risk clustered in people with a combination of both low CRF and low MVPA. ${ }^{15,16}$ However, ST was not included in these analyses. On the other hand, associations between ST and cardio-metabolic biomarkers seem less pronounced when CRF was taken into account. $^{17}$

Additional insight into sedentary behavior, PA, and CRF and their interrelationship as risk factors for cardio-metabolic health may help to expand public health messages and policies aimed at preventing CVD. Therefore, the aim of this study was to examine the mutual independent and combined associations of ST, MVPA and CRF on cardiometabolic health and diabetes status. 


\section{Methods}

\section{Population}

We used data from The Maastricht Study, an observational prospective populationbased cohort study. The rationale and methodology have been described previously. ${ }^{18}$ In brief, the study focuses on the etiology, pathophysiology, complications and comorbidities of T2DM and is characterized by an extensive phenotyping approach. Eligible for participation were all individuals aged between 40 and 75 years and living in the southern part of the Netherlands. Participants were recruited through mass media campaigns and from the municipal registries and the regional Diabetes Patient Registry via mailings. Recruitment was stratified according to known T2DM status, with an oversampling of individuals with T2DM, for reasons of efficiency. The present report includes cross-sectional data from a selection of the first 3,451 participants, who completed the baseline survey between November 2010 and September 2013. Data were available for 1,993 participants after excluding participants that did not receive an accelerometer due to logistics $(n=673)$, with invalid accelerometer readings $(n=136)$, with unperformed $(n=282)$ or invalid $(n=144)$ CRF measurement, with missing data on cardio-metabolic outcome variables $(n=8)$, missing covariates $(n=195)$, and with type 1 diabetes, or other forms of diabetes $(n=20)$. The study complies with the Declaration of Helsinki and has been approved by the institutional medical ethical committee (NL31329.068.10) and the Minister of Health, Welfare and Sports of the Netherlands (Permit 131088-105234-PG). All participants gave written informed consent.

\section{Cardio-metabolic outcomes}

The following outcome measures were used: waist circumference, blood pressure, plasma levels of HDL-cholesterol, triglycerides and glucose, homeostatic model assessment insulin resistance (HOMA2-IR), the metabolic syndrome (MetS), and diabetes status. Details of assessment have been described previously. ${ }^{18}$ HOMA2-IR was calculated using the HOMA calculator available from https://www.dtu.ox.ac.uk. MetS was defined according to the ATP III guidelines. ${ }^{19}$ Medication use was assessed during a medication interview where generic name, dose, and frequency were registered. To determine diabetes status according to the World Health Organization 2006 criteria, ${ }^{20}$ all participants (except those who used insulin) underwent a standardized seven-point 75 gram oral glucose tolerance test after an overnight fast as described elsewhere. ${ }^{18}$ Participants were categorized as having normal glucose metabolism, prediabetes (fasting plasma glucose $6.1-6.9 \mathrm{mmol} / \mathrm{L}$ or $2 \mathrm{~h}$ plasma glucose $\geq 7.8-<11.1 \mathrm{mmol} / \mathrm{L}$ ), or T2DM (fasting plasma glucose $\geq 7.0 \mathrm{mmol} / \mathrm{L}$ and/or $2 \mathrm{~h}$ plasma glucose $\geq 11.1$ $\mathrm{mmol} / \mathrm{L})$. Participants taking glucose lowering medication were also considered as having T2DM. 


\section{Accelerometry: sedentary behaviour and HPA}

Daily activity levels were measured using the activPAL3 ${ }^{\mathrm{TM}}$ physical activity monitor (PAL Technologies, Glasgow, UK). Participants were asked to wear the accelerometer for 8 consecutive days, without removing it at any time. Data were uploaded using the activPAL software and processed using customized software written in MATLAB R2013b (MathWorks, Natick, MA, USA). Data from the first day were excluded from the analysis. In addition, data from the final wear day providing $\leq 14$ waking hours of data were excluded from the analysis. Participants were included if they provided at least 1 valid day ( $\geq 10 \mathrm{~h}$ of waking data).

The total amount of ST was calculated as the mean time spent in a sedentary position during waking time per day. The method used to determine waking time has been described elsewhere. ${ }^{21}$ The total amount of physical activity was calculated as the mean time stepping during waking time per day. Further, physical activity (stepping time) was classified as higher intensity physical activity (HPA) when step frequency $>110$ steps/min.22

\section{Cardio respiratory fitness (CRF)}

As an objective measure of $\mathrm{CRF}$, estimated maximum power output adjusted for body mass (Wmax kg-1) was used. Wmax was estimated from a graded sub-maximal exercise protocol performed on a cycle ergometer system (CASETM version 6.6 in combination with e-bike, GE Healthcare, Milwaukee, WI, USA). For safety reasons, participants with recent or manifest cardiovascular complications were excluded from the exercise test. The protocol has been describe in detail elsewhere. ${ }^{13}$

\section{Covariates}

Questionnaires were used to collect information on age (in years), sex, educational level (low, middle, and high), smoking behavior (non-smoker, former smokers, and current smokers), alcohol consumption (non-consumers, low-consumers (women $\leq 7$ glasses per week; men $\leq 14$ glasses per week), and high-consumers (women $>7$ glasses per week; men > 14 glasses per week)), cardio vascular disease history, mobility limitations (defined as having difficulty walking $500 \mathrm{~m}$ or climbing the stairs), and energy intake (calculated as the mean energy intake (kcal) per day from a frequent food questionnaire). Percentage of body fat was calculated with the Siri equation after estimating body density from skinfold thickness at four sites (supra-iliaca, sub-scapula, biceps, and triceps). ${ }^{23}$

\section{Statistical analyses}

First, descriptives were provided as means $( \pm \mathrm{SD})$ for normally distributed variables, median [25-75\%] for skewed variables or percentages for categorical variables. Second, linear regression analyses were performed to assess the independent association of ST, 
HPA, and CRF with individual cardio-metabolic outcome measures. (Multinomial) logistic regression analyses were performed for the outcomes MetS and diabetes status. Associations in model 1 were adjusted for age, sex, waking time, education level, smoking status, alcohol consumption, mobility limitation, cardiovascular disease history, and energy intake. Outcome glucose metabolism was additionally adjusted for body fat percentage, antihypertensive and lipid modifying medication. Associations in models $2 \mathrm{a}, 2 \mathrm{~b}$, and $2 \mathrm{c}$ were additionally adjusted for ST, HPA and CRF, respectively. To examine the relative importance of ST, HPA, and CRF for cardio-metabolic outcomes, ST, HPA, and CRF were expressed per one standard deviation. Third, combined associations of ST-CRF and HPA-CRF with the cardio-metabolic outcome measures were analyzed. In this analysis, CRF (Wmax kg-1) was categorized into tertiles (low, medium, and high fit) based on sex and age; (40-49, 50-59, 60-69, and $>70$ years). Further, the proportions of daily HPA and ST were categorized into sex-specific tertiles (low, medium, and high). For men, tertile cut points were $59 \%$ and $67 \%$ for ST and $1.0 \%$ and $2.3 \%$ for HPA. For women, tertile cut points were $52 \%$ and $60 \%$ for ST and $1.9 \%$ and $3.3 \%$ for HPA. Tertiles of CRF and HPA and tertiles of CRF and ST were combined into 9 subgroups. For each subgroup, the odds for MetS and prediabetes and T2DM were calculated. Covariates were the same as in model 1 described above. In addition, the adjusted means of subgroups based on CRF and HPA were adjusted for ST. The adjusted means of subgroups based on CRF and ST were adjusted for HPA. In additional analyses, adjusted means of individual markers of cardio metabolic health were calculated using general linear models.

In all analyses men and women were analyzed together, as no interaction effect of sex was observed. In additional analyses participants with mobility limitations $(n=341)$ were excluded in order to minimize risk for reverse causation.

\section{Results}

The characteristics of the study population are described in table 5.1.The MetS was present in $36.0 \%$ of the population. Further, $58.8 \%$ of the population had normal glucose metabolism, 15.7\% had prediabetes and 25.4\% had T2DM. Mean CRF was $2.1 \pm 0.6$ $\mathrm{W} \mathrm{kg}-1$. Based on the accelerometry data, an average day consisted of $15.7 \pm 0.9$ hours waking time and during this waking time $9.3 \pm 1.6$ hours were spent in sedentary pos-

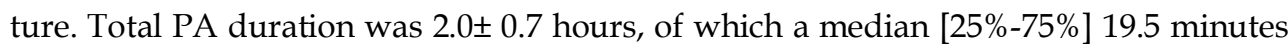
[9.9-32.0] were spent in HPA. 
Table 5.1: characteristics of the study population $(\mathrm{N}=1,993)$

\begin{tabular}{lrl} 
Characteristic & & \\
\hline Age (years) & 59.8 & $(8.1)$ \\
Sex (\% male) & 49.4 & \\
Educational level (\% high) & 39.8 & \\
Smoking status (\% current) & 12.1 & \\
Alcohol consumption (\% high) & 25.3 & \\
Mobility limitation (\%) & 17.1 & \\
History of CVD (\% yes) & 14.0 & \\
BMI (kg/m2) & 26.7 & $(4.3)$ \\
Waist circumference (cm) & 94.7 & $(13.1)$ \\
Systolic BP (mmHg) & 134.4 & $(17.7)$ \\
Diastolic BP (mmHg) & 76.2 & $(9.7)$ \\
Triglycerides (mmol/L) & 1.2 & {$[0.9-1.7]$} \\
HDL-cholesterol (mmol/L) & 1.6 & $(0.5)$ \\
Fasting glucose (mmol/L) & 5.5 & {$[5.0-6.4]$} \\
HOMA-IR* & 1.4 & {$[1.0-2.1]$} \\
Glucose lowering medication (\%) & 20.2 & \\
Antihypertensive medication (\%) & 37.1 & \\
Lipid lowering medication (\%) & 34.4 & \\
The metabolic syndrome (\%) & 36.3 & \\
Glucose metabolism status: & & \\
- normal (\%) & 58.8 & \\
- prediabetes (\%) & 15.7 & \\
- type 2 diabetes mellitus (\%) & 25.5 & \\
Valid days (N) & 6.3 & $(1.2)$ \\
Waking time (h/day) & 15.7 & $(0.9)$ \\
Sedentary time (h/day) & 9.3 & $(1.6)$ \\
Total PA (h/ day) & 2.0 & $(0.7)$ \\
Higher intensity PA (min/day) & 19.5 & {$[9.9-32.0]$} \\
CRF (Wmax kg- ${ }^{*}$ ) & 2.1 & $(0.6)$ \\
\hline & & \\
\hline
\end{tabular}

CVD, cardiovascular disease; BMI, body mass index; PA, physical activity; CRF, cardiorespiratory fitness. Values expressed as mean (SD), median [25-75\%], or percentages. ${ }^{*} \mathrm{~N}=1,893$

In table 5.2, the mutually independent associations of ST, HPA, and CRF with individual markers of cardio-metabolic health are provided. A larger amount of ST was associated with statistically significantly larger waist circumference, lower HDL, higher TG, higher glucose, and higher HOMA2-IR independent of HPA and CRF. More HPA was associated with smaller waist circumference, higher HDL, lower TG, and lower HOMA2-IR after adjustment for ST and CRF. Higher CRF was associated with smaller waist circumference, lower diastolic blood pressure, higher HDL, lower levels of TG, lower glucose, and lower HOMA2-IR.

Table 5.3 provides the independent associations between ST, HPA, and CRF and the likelihood of MetS, prediabetes and T2DM. As observed in models $2 b$ and 2c, larger amount of ST was associated with greater odds for MetS independent of HPA and CRF and with greater odds for T2DM independent of HPA and CRF. More time spent in 


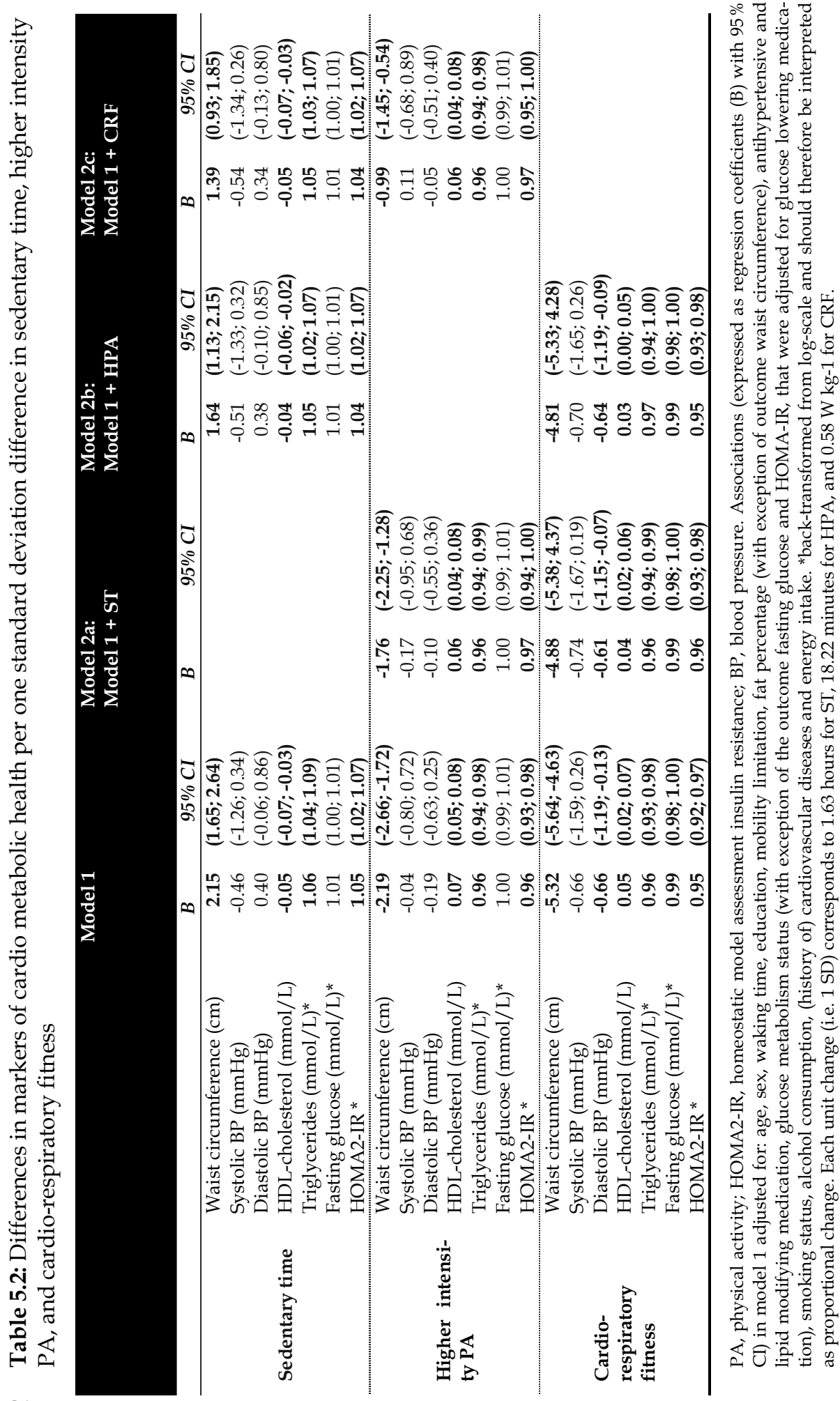




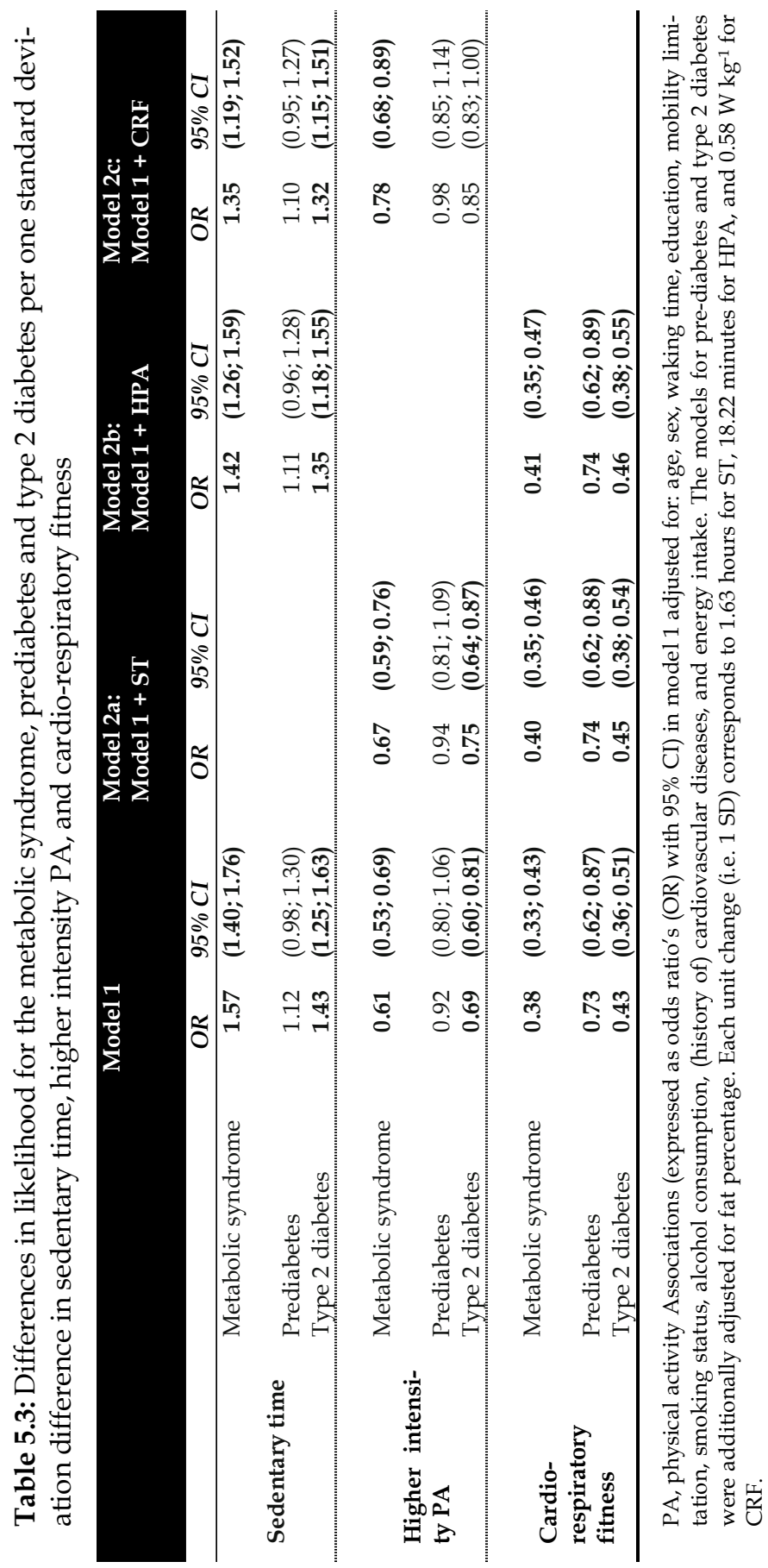


HPA was associated with lower odds for MetS independent of ST and CRF. In addition HPA was associated with lower odds for T2DM independent of ST (model 2a), but not independent of CRF (model 2c). Higher CRF was associated with lower odds for MetS independent of ST and HPA with lower odds for prediabetes independent of ST and HPA and with lower odds for T2DM independent of ST and HPA (models $2 \mathrm{~b}$ and 2c). For both ST and HPA no indication for interaction with CRF was found (when the terms $\mathrm{ST}^{*} \mathrm{CRF}$ and $\mathrm{HPA}^{*} \mathrm{CRF}$ were added to the models p-value $>0.30$ ).

Figure 5.1 shows the associations of the combined tertiles of CRF and HPA (left panel) and CRF and ST (right panel) with MetS. Compared to people with high CRF - high HPA (=reference) all other subgroups with medium or low CRF had higher odds for MetS, with the greatest odds in the subgroup low CRF - low HPA, OR=5.73(3.84; 8.56). Odds for MetS was greater in people with low CRF - high HPA, OR=4.46 (2.74; 7.26) than in those high CRF - low HPA, OR= $1.64(0.99 ; 2.72)$. When analyzing the contribution of ST, people with higher ST and with medium or low CRF had greater odds for MetS compared to those with high CRF - low ST (=reference). The highest odds was seen in those with low CRF - high ST, OR=9.22(5.74; 14.80). Also, compared to people with high CRF - low ST, those with high CRF - high ST had greater odds for MetS, $\mathrm{OR}=2.93$ (1.72; 4.99). Overall, the contribution of CRF seemed larger in comparison to ST as odds for MetS in the subgroup of low CRF - low ST (OR=5.62 $(3.35 ; 9.41))$ was greater than the odds of high CRF- high ST.

The left panel of figure 5.2 shows the associations of the combined tertiles of CRF and HPA with diabetes status (prediabetes top panel; T2DM bottom panel). Compared to the subgroup high CRF - high HPA (=reference) the odds for prediabetes was higher in persons with low CRF and medium CRF - low HPA. In addition, the odds for T2DM was greater in those with medium and low CRF (with the exception of medium CRF High HPA), with greatest odds in low CRF - low HPA, OR=6.42 (3.95; 10.45). In participants with high CRF, all levels of HPA had similar odds for T2DM. The right panel of figure 2 shows the associations with of the combined tertiles of CRF and ST with diabetes status. Compared to people with high CRF - low ST (=reference), the odds for prediabetes was greater in all low and medium CRF subgroups. In persons with high CRF, people in the highest tertile of ST (high CRF - high ST) had increased odds for prediabetes as well: $\mathrm{OR}=1.94(1.01 ; 3.72)$. Using the same comparisons, all low and medium CRF subgroups had greater odds for T2DM and persons with low CRF - high ST had the greatest odds for T2DM, OR=8.38 $(4.83 ; 14.55)$. People with high CRF - high ST had increased odds for T2DM as well, OR=2.21 $(1.17 ; 4.17)$ but this was lower than the odds of persons with low CRF - low ST: OR=5.62 $(3.35 ; 9.41)$, suggesting that the contribution of CRF was larger in comparison to ST. 

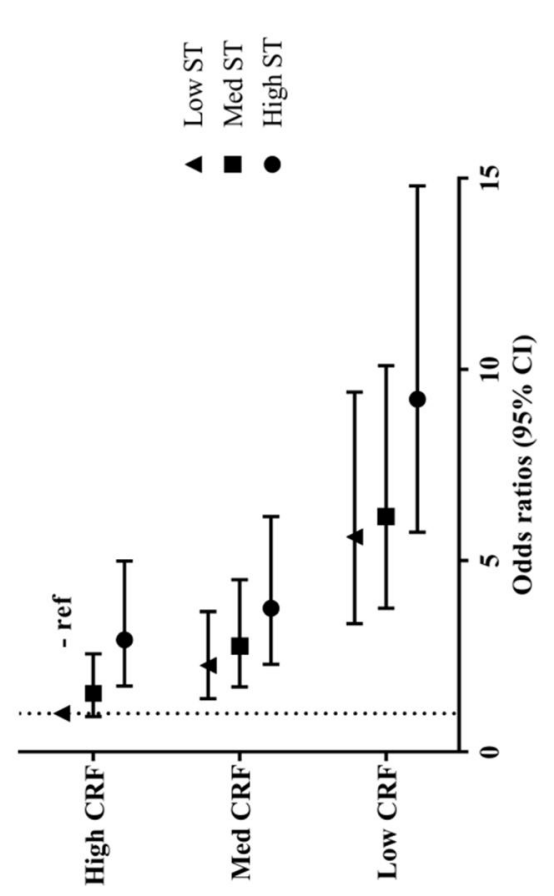

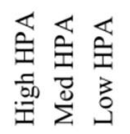

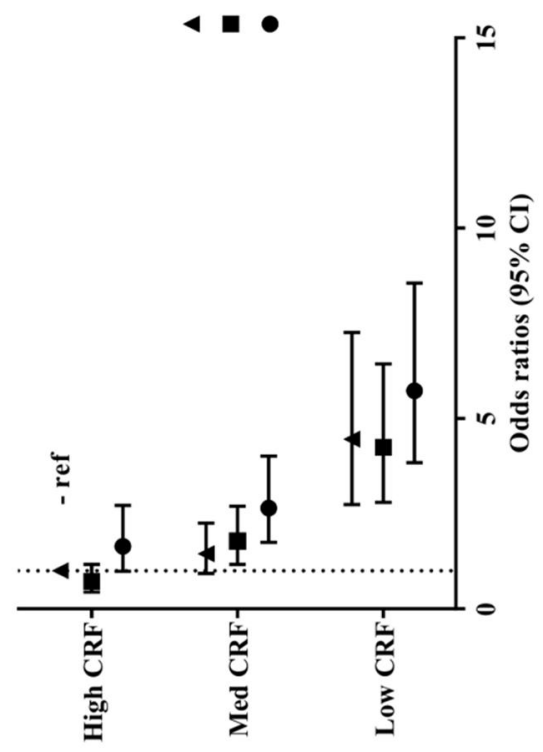

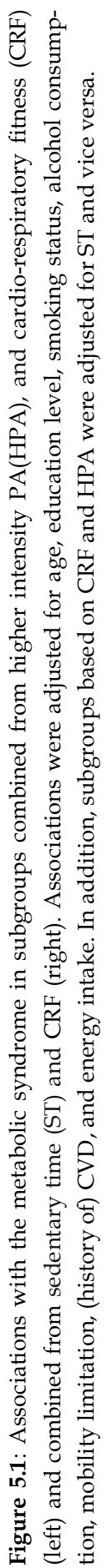



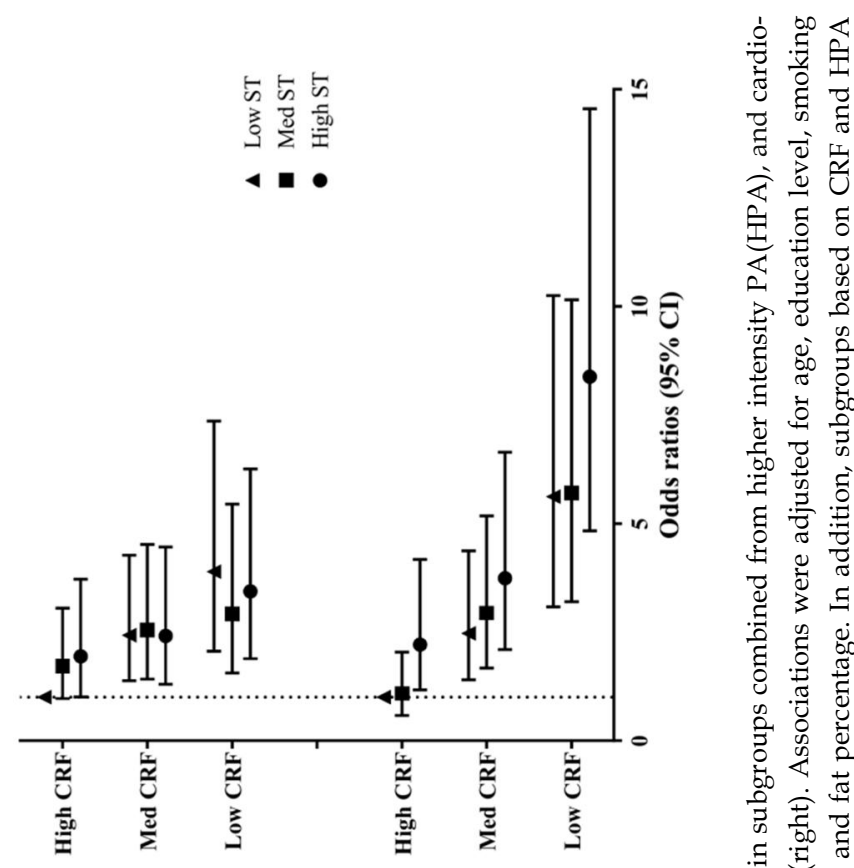

究芯

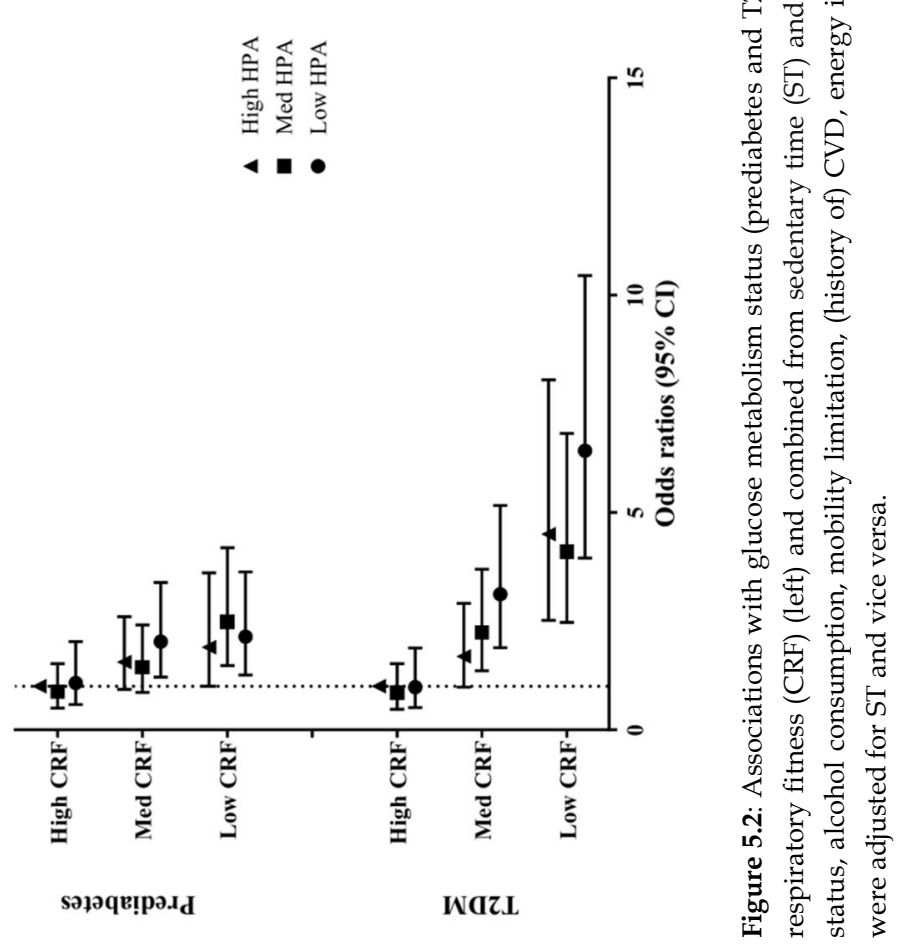


In supplemental figures S5.1 and S5.2, the associations of combined tertiles of CRF and ST and CRF and HPA with individual markers of cardio-metabolic health are provided. These results demonstrated also that the largest differences in cardio-metabolic markers are between low and high CRF. In addition, even at high levels of CRF, lower levels of HPA and higher levels of ST were associated with lower HDL and higher TG.

In additional analyses all participants with mobility limitations $(n=341)$ were excluded from analyses. This did not noticeably affect any of the associations presented in tables 5.1 and 5.2 and figures 5.1 and 5.2 (data not tabulated).

\section{Discussion}

Several studies have highlighted the importance of sedentary behavior, physical activity and fitness for cardio-metabolic health, but when developing preventive strategies these factors should not be viewed in isolation. This cross-sectional study is, to our knowledge, the first to examine the independent and combined associations of ST, HPA, and CRF with cardio-metabolic health. Our results showed that ST, HPA, and CRF were all mutually independently associated with markers of cardio-metabolic health, MetS and T2DM.

In relative terms, the strength of the associations of CRF on MetS and diabetes status appeared to be stronger than the associations HPA and ST (table 2). When analyzing the combined associations of ST, HPA and CRF, we observed that, overall, an individual with high HPA or low ST but in combination with low CRF, was at higher risk for a unfavorable cardio-metabolic risk profile than an individual with low HPA or high ST in combination with high CRF (figures 1 and 2). In addition, more frequently engaging in HPA did not seem to compensate the harmful outcomes associated with having a low CRF, as there were no, or only relatively small differences in the odds for MetS, prediabetes and T2DM between persons with high vs low HPA. Similarly, the harmful outcomes associated with low ST versus high ST were outweighed by the harmful outcomes associated with having lower level of CRF. We observed the highest odds for MetS (OR 9.22), prediabetes (OR 3.44) and T2DM (OR 8.38) in persons with a low CRF combined with a high ST.

Results of our study indicate that ST, independent of HPA and CRF, is associated with poor cardio-metabolic health and T2DM. Associations between high ST and poor (markers of) cardio-metabolic health, independent of HPA have been described previously.5,6 Recently, we reported that in The Maastricht Study cohort each extra hour of ST was associated with a $22 \%$ increased odds for T2DM and a $39 \%$ increased odds for MetS. ${ }^{24}$ Associations between objectively measured ST and poor cardio-metabolic health, independent of CRF, have been reported in some, ${ }^{25,26}$ but not all earlier 
studies.17,27,28 In the combined associations of CRF and ST, we showed that odds for MetS and T2DM were also greater in the subgroup high CRF-high ST. These results indicate that even individuals with high CRF may be at increased risk for metabolic diseases due to prolonged sitting time.

In line with our results, others have reported beneficial associations of HPA on cardiometabolic risk, independent of CRF.15,26,29-34 The studies comparing effect sizes of the associations of HPA and CRF with health reported a larger effect size of CRF as well, 26,29,32,33 but combined associations of HPA-CRF with cardio-metabolic outcomes have been described scarcely. One study observed that individuals with a combination of high CRF-low HPA had a more healthy cardio-metabolic risk profile than those with a combination of low CRF- high HPA. ${ }^{15}$ A prospective study examining the incidence of T2DM reported similar results. ${ }^{34}$ This seems in agreement with our results. In our study, compared to high CRF-high HPA, all HPA subgroups with low CRF had higher odds for MetS, prediabetes and T2DM, regardless of HPA. A high level of HPA was sufficient to 'counteract' the detrimental associations in subgroups with medium CRF only and did not seem to have additional benefit in persons with high CRF in our analyses (as seen in figure 2).

The relative importance of HPA and ST versus CRF for cardio-metabolic health should be discussed in light of the mediating effects of CRF. For example, CRF explained 73\% of the association between MVPA and metabolic risk in a recent study by Knaeps et al. ${ }^{35}$ and an even greater mediating effect of CRF was reported in another mediation analyses. ${ }^{32}$ Biological pathways through which HPA affects cardio-metabolic health could therefore be similar to CRF. In addition, recent studies have observed an association between high ST and lower CRF,11,12 implying that also the association between ST and cardio-metabolic health could be partly mediated through lower CRF. ${ }^{36}$ Nonetheless, results from our joint analyses showed an elevated risk for MetS and T2DM in the high CRF - high ST subgroup, suggesting that other mechanisms are involved as well and that a high CRF may not be sufficient to 'counteract' the deleterious health outcomes associated with sedentary behavior.

Globally, daily amount of ST is increasing and at the same time, amount of MVPA is decreasing. ${ }^{3}$ Presently, people generally spent the majority of the day in sedentary behavior. Thus, although the strength of the associations of ST was relatively small compared to that of CRF, reducing ST potentially has a great impact on public health due to its high prevalence. Whether sittig time should be reduced by increasing the daily amount of light intensity physical activities or that relative brief periods of MVPA are sufficient to improve cardio-metabolic risk is still debated. ${ }^{7}$ 
Cross-over studies in sedentary subjects with and without T2DM suggested that sedentary behavior has negative cardio-metabolic effects which are independent of changes in energy balance. Replacing ST with regular short bouts of light intensity physical activities (which presumably have a relatively small effect on CRF) had in these studies more positive cardio-metabolic effects than HPA. ${ }^{37,38}$ Reduction in activity of AMP activated protein kinase (AMPK) and lipoprotein lipase (LPL) activity, due to contractile inactivity of skeletal muscles, could be important underlying mechanisms of the effects of prolonged ST on glucose and lipid metabolism. ${ }^{39}$ Physical activity is usually associated with an increase of blood flow induced shear stress on the vascular endothelium, which plays an important role in maintaining vascular homeostasis. Endothelial dysfunction, a key event in the development of CVD, could therefore be another consequence of prolonged sedentary behavior. ${ }^{40}$ Since sedentary behavior research is a relatively young field, studies investigating biological mechanisms explaining detrimental effects of prolonged ST are warranted. Future work should also focus on doseresponse; what amount of sitting time is associated with a clinical relevant increase in risk and which levels of HPA and CRF are associated with clinical relevant lower risk for MetS and T2DM? In this study, low, medium and high CRF, ST and HPA were derived from data driven tertiles. Thus, the cut points between tertiles may not represent clinically relevant cut-off points.

Strengths of this study include the use of a posture based activity monitor to assess ST and HPA, and the objective measure of CRF. However, our results should also be interpreted in the light of some limitations. Importantly, interpretation of causality should be done with caution due to the cross-sectional nature of this study. For instance, people may change their behavior due to illness. However, we attempted to eliminate these influences by adjusting our analyses for mobility limitations. Moreover, repeating the analyses after excluding those with mobility limitations did not alter our findings. Further, HPA was based on step frequency, which may be less precise to determine PA intensity compared with methods based on acceleration data. However, the applied frequency of $>110$ steps/minute has been reported to correspond to a MET score > 3.0. ${ }^{22}$ Therefore, it may be interpreted as an approximation of MVPA. In addition, selection bias may have been introduced as a result from the exclusion criteria applied for the sub-maximal exercise test. Consequently participants with recent or current cardiovascular complications have been excluded. This may have resulted in a slight underestimation of true effect sizes as the study population is healthier than the general population. Finally, The Maastricht Study comprises mainly Caucasians from European decent with well controlled participants with T2DM. This limits generalizability to other populations. 
In conclusion, high ST, low HPA and low CRF were each associated with several markers of cardio-metabolic health and higher risk for MetS and T2DM independent of each other. Combinations of low CRF - low HPA and low CRF - high ST were associated with a particularly high risk of having MetS and T2DM. A shift from low to medium CRF was associated with greatest reduction in risk for having MetS and T2DM. Additionally, reducing ST as well as increasing HPA was associated with additive risk reductions and in relative terms the strengths of these associations were comparable. In order to improve cardiovascular risk and to prevent T2DM these data support the development of new strategies that reduce sedentary behavior as well as increase higher intensity physical activity. 


\section{References}

1. World Health Orginisation. Factsheet cardiovascular diseases.

http://www.who.int/mediacentre/factsheets/fs317/en/ (April 13, 2017).

2. Warburton DE, Nicol CW, Bredin SS. Health benefits of physical activity: the evidence. CMAJ 2006;174(6):801-9.

3. Ng SW, Popkin BM. Time use and physical activity: a shift away from movement across the globe. Obes. Rev. 2012;13(8):659-680.

4. Sedentary-Behaviour-Research-Network. (2102). Letter to the editor: standardized use of the terms "sedentary" and "sedentary behaviours". Appl. Physiol. Nutr. Metab 2012;37(3):540-542.

5. Brocklebank LA, Falconer CL, Page AS, Perry R, Cooper AR. Accelerometer-measured sedentary time and cardiometabolic biomarkers: A systematic review. Prev. Med. 2015;76:92-102.

6. Wilmot EG, Edwardson CL, Achana FA, Davies MJ, Gorely T, Gray LJ, Khunti K, Yates T, Biddle SJ. Sedentary time in adults and the association with diabetes, cardiovascular disease and death: systematic review and meta-analysis. Diabetologia 2012;55(11):2895-2905.

7. Young DR, Hivert M-F, Alhassan S, Camhi SM, Ferguson JF, Katzmarzyk PT, Lewis CE, Owen N, Perry CK, Siddique J. Sedentary Behavior and Cardiovascular Morbidity and Mortality: A Science Advisory From the American Heart Association. Circulation 2016;134(13):e262-e279.

8. Lee DC, Artero EG, Sui X, Blair SN. Review: Mortality trends in the general population: the importance of cardiorespiratory fitness. J. Psychopharmacol. 2010;24(4 suppl):27-35.

9. Kodama S, Saito K, Tanaka S, Maki M, Yachi Y, Asumi M, Sugawara A, Totsuka K, Shimano H, Ohashi Y, Yamada N, Sone H. Cardiorespiratory Fitness as a Quantitative Predictor of All-Cause Mortality and Cardiovascular Events in Healthy Men and Women A Meta-analysis. Jama-Journal of the American Medical Association 2009;301(19):2024-2035.

10. Bouchard C, Rankinen T. Individual differences in response to regular physical activity. Med. Sci. Sports Exerc. 2001;33(6 Suppl):S446-51; discussion S452-3.

11. Kulinski JP, Khera A, Ayers CR, Das SR, de Lemos JA, Blair SN, Berry JD. Association between cardiorespiratory fitness and accelerometer-derived physical activity and sedentary time in the general population. Mayo Clin. Proc. 2014;89(8):1063-1071.

12. Prince SA, Blanchard CM, Grace SL, Reid RD. Objectively-measured sedentary time and its association with markers of cardiometabolic health and fitness among cardiac rehabilitation graduates. Eur J Prev Cardiol 2015;23(8):818-825.

13. van der Velde JHPM, Koster A, van der Berg JD, Sep SJ, van der Kallen CJ, Dagnelie PC, Schram MT, Henry RM, Eussen SJ, van Dongen MC, Stehouwer CD, Schaper NC, Savelberg HHCM. Sedentary Behavior, Physical Activity, and Fitness-The Maastricht Study. Med. Sci. Sports Exerc. 2017.

14. Després J-P. Physical Activity, Sedentary Behaviours, and Cardiovascular Health: When Will Cardiorespiratory Fitness Become a Vital Sign? Can. J. Cardiol. 2016;32(4):505-513.

15. Ekblom-Bak E, Hellenius ML, Ekblom O, Engstrom LM, Ekblom B. Independent associations of physical activity and cardiovascular fitness with cardiovascular risk in adults. Eur. J. Cardiovasc. Prev. Rehabil. 2010;17(2):175-80.

16. Franks PW, Ekelund U, Brage S, Wong M-Y, Wareham NJ. Does the association of habitual physical activity with the metabolic syndrome differ by level of cardiorespiratory fitness? Diabetes Care 2004;27(5):1187-1193.

17. Shuval K, Finley CE, Barlow CE, Gabriel KP, Leonard D, Kohl HW. Sedentary behavior, cardiorespiratory fitness, physical activity, and cardiometabolic risk in men: the cooper center longitudinal study. Mayo Clin. Proc. 2014;89(8):1052-1062.

18. Schram MT, Sep SJ, van der Kallen CJ, Dagnelie PC, Koster A, Schaper N, Henry RM, Stehouwer $\mathrm{CD}$. The Maastricht Study: an extensive phenotyping study on determinants of type 2 diabetes, its complications and its comorbidities. Eur. J. Epidemiol. 2014;29(6):439-451.

19. Alberti K, Eckel RH, Grundy SM, Zimmet PZ, Cleeman JI, Donato KA, Fruchart J-C, James WPT, Loria CM, Smith SC. Harmonizing the metabolic syndrome a joint interim statement of the international diabetes federation task force on epidemiology and prevention; national heart, lung, and blood institute; American heart association; world heart federation; international 
atherosclerosis society; and international association for the study of obesity. Circulation 2009;120(16):1640-1645.

20. World Health Organisation. Definition and Diagnosis of Diabetes Mellitus and Intermediate Hyperglycemia: Report of a WHO consultation. Geneva: World Health Organization. 2006.

21. van der Berg JD, Willems PJ, van der Velde JHPM, Savelberg HHCM, Schaper NC, Schram MT, Sep SJ, Dagnelie PC, Bosma H, Stehouwer CDA. Identifying waking time in 24-h accelerometry data in adults using an automated algorithm. J. Sports Sci. 2016;34(19):1867-73.

22. Tudor-Locke C, Rowe DA. Using cadence to study free-living ambulatory behaviour. Sports Med. 2012;42(5):381-398.

23. Siri WE. Body composition from fluid spaces and density: analysis of methods. Techniques for measuring body composition 1961;61:223-44.

24. van der Berg JD, Stehouwer CD, Bosma H, van der Velde JH, Willems PJ, Savelberg HH, Schram MT, Sep SJ, van der Kallen CJ, Henry RM. Associations of total amount and patterns of sedentary behaviour with type 2 diabetes and the metabolic syndrome: The Maastricht Study. Diabetologia 2016;59(4):709-718.

25. Greer AE, Sui X, Maslow AL, Greer BK, Blair SN. The effects of sedentary behavior on metabolic syndrome independent of physical activity and cardiorespiratory fitness. J. Phys. Act. Health 2015;12(1):68-73.

26. Ekblom O, Ekblom-Bak E, Rosengren A, Hallsten M, Bergstrom G, Borjesson M. Cardiorespiratory Fitness, Sedentary Behaviour and Physical Activity Are Independently Associated with the Metabolic Syndrome, Results from the SCAPIS Pilot Study. PLoS ONE 2015;10(6):e0131586.

27. van der Velde JHPM, Savelberg HHCM, Schaper NC, Koster A. Moderate activity and fitness, Not sedentary time, Are independently associated with cardio-metabolic risk in US adults aged 18-49. Int. J. Environ. Res. Public. Health 2015;12(3):2330-2343.

28. Nauman J, Stensvold D, Coombes JS, Wisløff U. Cardiorespiratory Fitness, Sedentary Time, and Cardiovascular Risk Factor Clustering. Med. Sci. Sports Exerc. 2016;48(4):625-632.

29. Minder CM, Shaya GE, Michos ED, Keenan TE, Blumenthal RS, Nasir K, Carvalho JA, Conceição RD, Santos RD, Blaha MJ. Relation between self-reported physical activity level, fitness, and cardiometabolic risk. Am. J. Cardiol. 2014;113(4):637-643.

30. O'Donovan G, Hillsdon M, Ukoumunne OC, Stamatakis E, Hamer M. Objectively measured physical activity, cardiorespiratory fitness and cardiometabolic risk factors in the Health Survey for England. Prev. Med. 2013;57(3):201-205.

31. Schmidt MD, Cleland VJ, Thomson RJ, Dwyer T, Venn AJ. A comparison of subjective and objective measures of physical activity and fitness in identifying associations with cardiometabolic risk factors. Ann. Epidemiol. 2008;18(5):378-386.

32. Sassen B, Cornelissen VA, Kiers H, Wittink H, Kok G, Vanhees L. Physical fitness matters more than physical activity in controlling cardiovascular disease risk factors. Eur. J. Cardiovasc. Prev. Rehabil. 2009;16(6):677-683.

33. Williams PT. Physical fitness and activity as separate heart disease risk factors: a meta-analysis. Med. Sci. Sports Exerc. 2001;33(5):754-761.

34. Sieverdes JC, Sui X, Lee D-c, Church TS, McClain A, Hand GA, Blair SN. Physical activity, cardiorespiratory fitness and the incidence of type 2 diabetes in a prospective study of men. $B r$. J. Sports Med. 2010;44(4):238-244.

35. Knaeps S, Lefevre J, Wijtzes A, Charlier R, Mertens E, Bourgois JG. Independent Associations between Sedentary Time, Moderate-To-Vigorous Physical Activity, Cardiorespiratory Fitness and Cardio-Metabolic Health: A Cross-Sectional Study. PLoS ONE 2016;11(7):e0160166.

36. Knaeps S, Bourgois JG, Charlier R, Mertens E, Lefevre J, Wijndaele K. Ten-year change in sedentary behaviour, moderate-to-vigorous physical activity, cardiorespiratory fitness and cardiometabolic risk: independent associations and mediation analysis. Br. J. Sports Med. 2016:bjsports-2016-096083.

37. Duvivier BM, Schaper NC, Hesselink MK, van Kan L, Stienen N, Winkens B, Koster A, Savelberg $\mathrm{HH}$. Breaking sitting with light activities vs structured exercise: a randomised crossover study demonstrating benefits for glycaemic control and insulin sensitivity in type 2 diabetes. Diabetologia 2017;60(3):490-498.

38. Duvivier BM, Schaper NC, Bremers MA, van Crombrugge G, Menheere PP, Kars M, Savelberg HH. Minimal Intensity Physical Activity (Standing and Walking) of Longer Duration Improves Insulin 
Action and Plasma Lipids More than Shorter Periods of Moderate to Vigorous Exercise (Cycling) in Sedentary Subjects When Energy Expenditure Is Comparable. PLoS ONE 2013;8(2):e55542.

39. Hamilton MT, Hamilton DG, Zderic TW. Role of low energy expenditure and sitting in obesity, metabolic syndrome, type 2 diabetes, and cardiovascular disease. Diabetes 2007;56(11):2655-67.

40. Thosar SS, Johnson BD, Johnston JD, Wallace JP. Sitting and endothelial dysfunction: the role of shear stress. Medical Science Monitor 2012;18(12):REV173-REV180. 
Supplement to chapter 5 

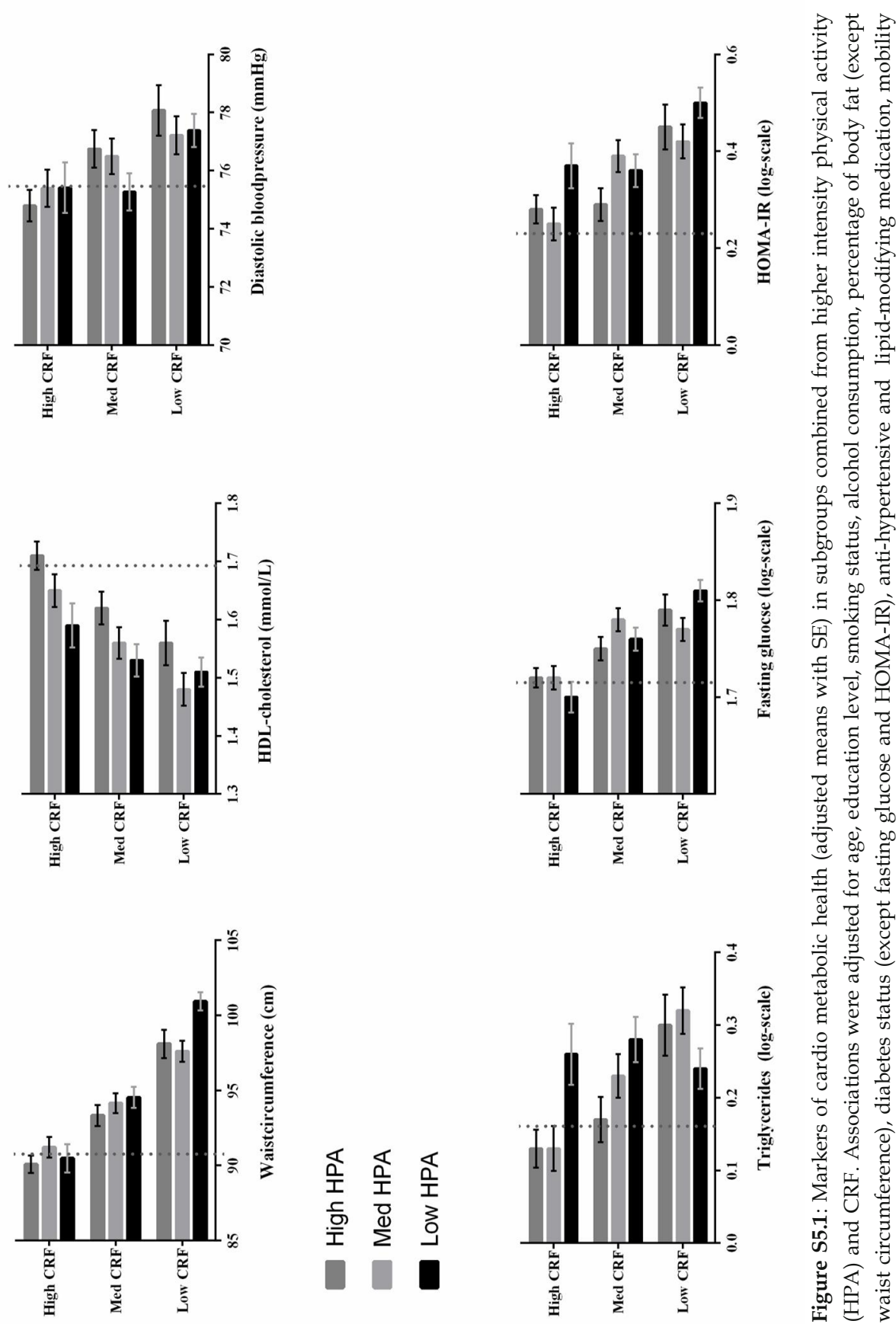

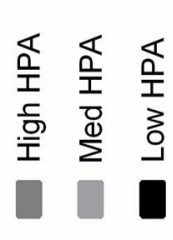

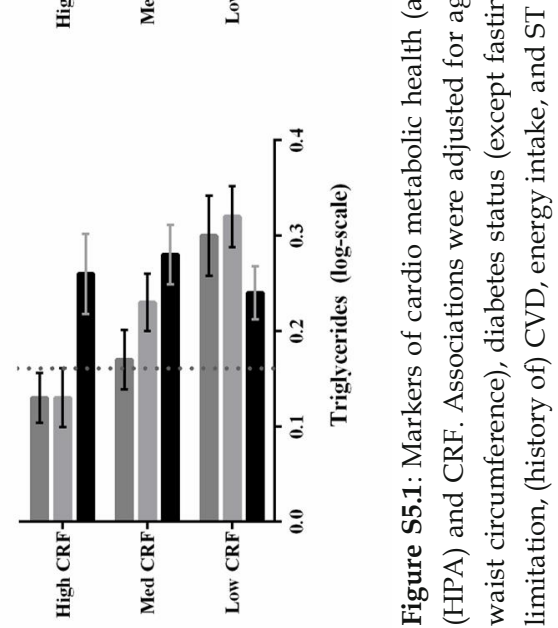



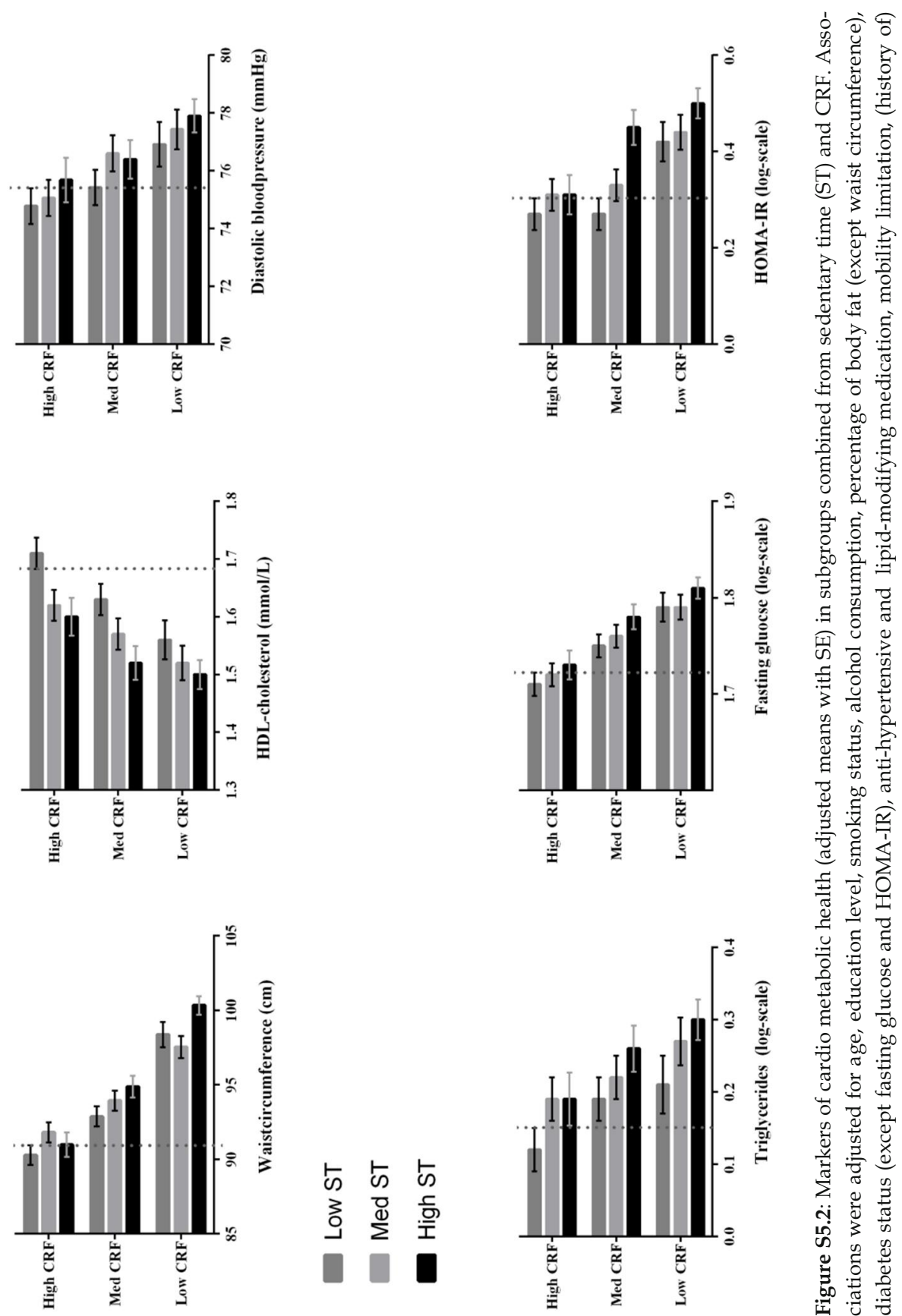

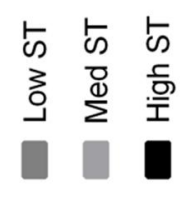
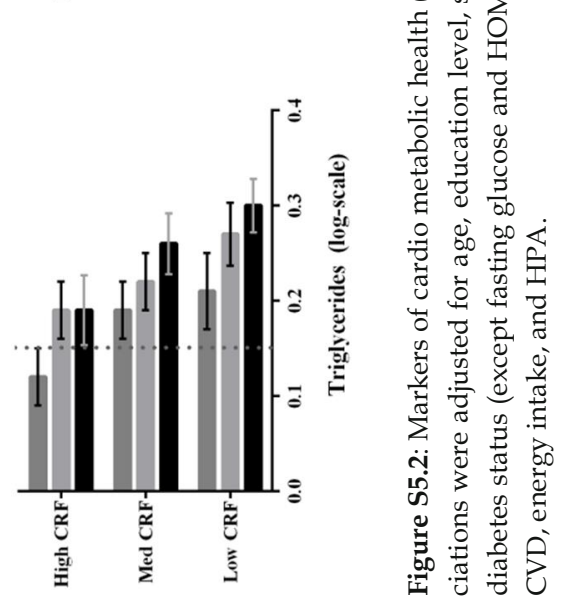


\section{Chapter 6}

\section{Replacement effects of sedentary time on metabolic outcomes}

\section{- The Maastricht Study -}

Julianne D van der Berg, Jeroen HPM van der Velde, Ellis AC de Waard, Hans Bosma, Hans HCM Savelberg, Nicolaas C Schaper, Joop PW van den Bergh, Piet PMM Geusens, Miranda T Schram, Simone JS Sep, Carla JH van der Kallen, Ronald MA Henry, Pieter C Dagnelie, Simone JPM Eussen, Martien CJM van Dongen, Sebastian Köhler Abraham A Kroon, Coen DA Stehouwer, Annemarie Koster 


\section{Abstract}

Background: Sedentary time has been associated with detrimental health effects, so in some countries guidelines to reduce sedentary time have been developed. As reducing sedentary time inevitably results in more non-sedentary time, effects of this reduction may depend on the activity with which it is replaced.

Aim: To examine associations of theoretical reallocations of sedentary time to standing or stepping with cardio-metabolic outcomes and type 2 diabetes.

Methods: We included 2,213 participants (52\% men, age (mean \pm SD): $60.0 \pm 8.1$ years) of The Maastricht Study who were asked to wear an accelerometer 24h/day for a week. We calculated daily sedentary, standing, and stepping time. An isotemporal substitution modelling approach was applied to examine effects on waist circumference, BMI, cholesterol, triacylglycerol, glucose, and insulin levels, metabolic syndrome and type 2 diabetes.

Results: Replacement of sedentary time (30 $\mathrm{min} /$ day) with stepping was associated with lower odds for metabolic syndrome $(\mathrm{OR}=0.72(95 \% \mathrm{CI}=0.66 ; 0.78))$ and type 2 diabetes $(\mathrm{OR}=0.79(0.72 ; 0.87))$, more favourable waist circumference $(\mathrm{B}=-1.42(-1.78$; 1.06)) and BMI (B = -0.48 (-0.62; -0.35)), and improved cholesterol, triacylglycerol, glucose, and insulin levels. Replacing sedentary time with standing was associated with lower odds for metabolic syndrome and type 2 diabetes, and favourable outcomes in waist circumference, cholesterol, triacylglycerol and insulin levels.

Conclusion: Theoretical replacements of sedentary time with non-sedentary time (both standing and stepping) were associated with lower odds for metabolic syndrome, type 2 diabetes, and beneficial metabolic outcomes. These results could be important for the general population, including those who cannot meet physical activity guidelines. Consideration should be given to developing recommendations for daily reallocating sedentary time. 


\section{Introduction}

Sedentary behavior, which refers to any waking behavior that is characterized by an energy expenditure $\leq 1.5$ metabolic equivalents (METs) while in a sitting or reclining position such as watching TV or using the computer, ${ }^{1}$ has been associated with several adverse health outcomes, including cardio-metabolic risk factors (e.g. waist circumference, cholesterol and triacylglycerol levels, insulin resistance), ${ }^{2,3}$ cardiovascular disease type 2 diabetes, and premature mortality. 4,5

Therefore, in some countries, health guidelines to reduce sedentary time have been developed by public health organizations and scientific institutions, ${ }^{6,7}$ and the Australian government has even published a sedentary behavior guideline. ${ }^{8}$ However, specific recommendations for the amount of sedentary time that should be reduced are lacking. To develop these recommendations, studies on the health effects of reducing sedentary time are needed. More specifically, as reducing sedentary time inevitably results in larger amounts of non-sedentary time which can vary from light physical activity (LPA) to vigorous physical activity, the effects of reducing sedentary time may depend on the activity with which it is replaced. Therefore, studies examining the effects of reduced sedentary time replaced with other types of behaviors are required.

An isotemporal substitution model (ISM) can be used to examine the effects of the theoretical replacement of sedentary time by non-sedentary time, like standing or stepping. ${ }^{9}$ Previous studies that have used an ISM approach demonstrated that a theoretical replacement of sedentary time with LPA or moderate to vigorous physical activity (MVPA) was associated with favorable health outcomes, including a reduction in BMI, 10-13 and waist circumference, ${ }^{10-12,14,15}$ and improved markers of insulin sensitivity, ${ }^{14,16}$ levels of cholesterol,12-15 triacylglycerol,12-15 and glucose.12,14,16

Although previous studies have used accelerometry data to assess sedentary time, in most studies sedentary time was solely based on acceleration data which cannot discriminate between postures. Consequently, standing time may have been misclassified as sedentary time or vice versa. ${ }^{17,18}$ Therefore, we used the activPAL accelerometer which classifies sedentary behavior (sitting or lying) using data on posture, as this has shown to be an accurate method for assessing sedentary behavior. ${ }^{17,19}$

The aim of this study was to examine the cross-sectional associations of a theoretical reallocation of sedentary behavior (sitting or lying) to standing or stepping with cardiometabolic outcomes, the metabolic syndrome and type 2 diabetes in 2,213 adults aged 40-75 years 


\section{Methods}

\section{Population}

We used data from The Maastricht Study, an observational prospective populationbased cohort study. The rationale and methodology have been described previously. ${ }^{20}$ In brief, the study focuses on the etiology, pathophysiology, complications and comorbidities of type 2 diabetes and is characterized by an extensive phenotyping approach. Eligible participants were individuals aged between 40 and 75 years and living in the southern part of the Netherlands. Participants were recruited through mass media campaigns and from the municipal registries and the regional Diabetes Patient Registry via mailings. Recruitment was stratified according to known type 2 diabetes status, with an oversampling of individuals with type 2 diabetes, for reasons of efficiency. This study included cross-sectional data from 3,451 participants, who completed the baseline survey between November 2010 and September 2013. The examinations of each participant were performed within a time window of three months. Participants with type 1 diabetes, latent auto-immune diabetes in adults (LADA), steroid-induced diabetes or diabetes after pancreatectomy $(n=41)$ were excluded. After further successively excluding participants who did not receive an accelerometer due to logistics $(n=668)$, whose accelerometer measurement failed $(n=135)$ or who had missing data on other measures $(n=394)$, a total of 2,213 participants were included in the present analyses.

The study was approved by the institutional medical ethical committee (NL31329.068.10) and the Minister of Health, Welfare and Sports of the Netherlands (permit 131088-105234-PG). All participants gave written informed consent.

\section{Cardio-metabolic outcomes}

The following cardio-metabolic outcomes were used: waist circumference, BMI, office blood pressure, HDL cholesterol, total-to-HDL cholesterol ratio, triacylglycerol, fasting glucose, $2 \mathrm{~h}$ post-load glucose, $\mathrm{HbA1c}$, fasting insulin, the metabolic syndrome, and type 2 diabetes. Waist circumference, weight and height were measured as described elsewhere (23). BMI was calculated as $\mathrm{kg} \bullet \mathrm{m}^{-2}$. Office blood pressure was determined three times on the right arm after a 10-minute rest period, using a non-invasive blood pressure monitor (Omron 705IT, Japan). All available measurements were used to calculate the average blood pressure. Fasting blood samples were used for laboratory assessment of total cholesterol, HDL cholesterol, triacylglycerol, glucose, HbA1c, and insulin levels (23). Total-to-HDL cholesterol ratio was calculated by dividing total cholesterol by HDL cholesterol. To determine $2 \mathrm{~h}$ post-load glucose and glucose metabolism status, all participants (except those who use insulin) underwent a standardized 75 g oral glucose tolerance test after an overnight fast as described elsewhere. ${ }^{20}$ The metabolic syndrome was defined according to the ATPIII guidelines by the presence of 3 or 
more of the following criteria: (1) waist circumference $\geq 102 \mathrm{~cm}$ for men and $\geq 88 \mathrm{~cm}$ for women; (2) serum triacylglycerol level $\geq 1.7 \mathrm{mmol} / \mathrm{L}$; (3) HDL cholesterol level $<1.03$ $\mathrm{mmol} / \mathrm{L}$ for men and $<1.30 \mathrm{mmol} / \mathrm{L}$ for women; (4) fasting glucose level $\geq 5.6 \mathrm{mmol} / \mathrm{L}$ or use of antidiabetic medications (insulin or oral agents); or (5) systolic blood pressure $\geq 130 \mathrm{mmHg}$ and/or diastolic blood pressure $\geq 85 \mathrm{mmHg}$, and/or use of antihypertensive medications. ${ }^{21}$ To determine type 2 diabetes, participants were categorized according to the World Health Organization's 2006 criteria, ${ }^{22}$ into normal glucose metabolism (NGM), impaired fasting glucose (fasting plasma glucose 6.1-6.9 mmol/L and $2 \mathrm{~h}$ plasma glucose (after glucose load) $<7.8 \mathrm{mmol} / \mathrm{L}$ ), impaired glucose tolerance (fasting plasma glucose $<7.0 \mathrm{mmol} / \mathrm{L}$ and $2 \mathrm{~h}$ plasma glucose $\geq 7.8-11.1 \mathrm{mmol} / \mathrm{L}$ ), or type 2 diabetes (fasting plasma glucose $\geq 7.0 \mathrm{mmol} / \mathrm{L}$ or $2 \mathrm{~h}$ plasma glucose $\geq 11.1 \mathrm{mmol} / \mathrm{L}$ ) . Impaired fasting glucose and impaired glucose tolerance were combined into prediabetes. Participants on diabetes medication and without the diagnosis of type 1 diabetes were also considered to have type 2 diabetes. For the analyses with type 2 diabetes as outcome measure, we used the categories: having type 2 diabetes and not having type 2 diabetes (NGM and prediabetes).

\section{Accelerometry}

Daily activity levels were measured using the activPAL3 ${ }^{\mathrm{TM}}$ physical activity monitor (PAL Technologies, Glasgow, UK), as described elsewhere. ${ }^{23}$ Participants were asked to wear the accelerometer for eight consecutive days, without removing it any time. Data from the first day were excluded from the analysis because participants performed physical function tests at the research center after the device was attached. In addition, data from the final wear day providing $\leq 14$ waking hours of data were excluded from the analysis. Participants were included if they provided at least 1 valid day ( $\geq 10$ h of waking data).

The total amount of sedentary time was based on the sedentary posture (sitting or lying), and calculated as the mean time spent in a sedentary position during waking time

per day. The method used to determine waking time has been described elsewhere. ${ }^{23}$ The total amount of standing time was based on the standing posture, and calculated as the mean time spent standing during waking time per day. The total amount of stepping was based on the stepping posture, and calculated as the mean time stepping during waking time per day.

\section{Covariates}

Covariates which were extracted from a questionnaire included sex, age, level of education, smoking status, alcohol consumption, energy intake, mobility limitation, and prevalent cardiovascular disease. Level of education was categorized into low, medium and high, and smoking status into never, former and current smoker. Alcohol con- 
sumption was categorized into non consumers, low consumers ( $\leq 7$ glasses per week for women and $\leq 14$ glasses/ week for men), and high consumers ( $>7$ glasses per week for women and >14 glasses/week for men). Energy intake was obtained from a food frequency questionnaire and calculated as the mean energy intake (kcal) per day. Mobility limitation was obtained from the Short Form Health Survey (SF-36) questionnaire and was defined as having difficulty walking $500 \mathrm{~m}$ or climbing the stairs. Prevalent CVD was defined as a self-reported history of myocardial infarction, cerebrovascular infarction or hemorrhage, or percutaneous artery angioplasty of, or vascular surgery on, the coronary, abdominal, peripheral or carotid arteries. The use of lipid-modifying, antihypertensive and glucose-lowering medication was assessed during a medication interview. Depression was assessed by the structured Mini-International Neuropsychiatric Interview (MINI). ${ }^{24}$ Lastly, glucose metabolism status (using the categories NGM, prediabetes, type 2 diabetes) was used as a covariate in all analyses except those with the metabolic syndrome and type 2 diabetes.

\section{Statistical analyses}

Characteristics of the total study population and according to sex-specific tertiles (to obtain equal distributions of men and women) of sedentary time, were summarized as means with standard deviations (SD) or as numbers and percentages. The variables total-to-HDL cholesterol ratio, triacylglycerol, fasting glucose, $2 \mathrm{~h}$ post-load glucose, fasting insulin, and $\mathrm{HbA} 1 \mathrm{c}$ had a skewed distribution, and were described using the median and the interquartile range. For the analyses these variables were transformed using natural logarithm.

Associations of sedentary time, standing, and stepping (all expressed per $30 \mathrm{~min}$ by dividing total time in minutes by 30 ) with the cardio-metabolic outcomes were examined for each type of behavior and each outcome separately, using linear regression analysis for the continuous variables and logistic regression analysis for the metabolic syndrome and type 2 diabetes. The associations were adjusted for sex, age, level of education, waking time, smoking status, alcohol consumption, energy intake, mobility limitation, prevalent cardiovascular disease, depression, and antihypertensive and lipid-modifying medication (the metabolic syndrome and type 2 diabetes were not adjusted for medication use). The associations were also adjusted for glucose metabolism status to take into account the oversampling of participants with type 2 diabetes in The Maastricht Study (the metabolic syndrome and type 2 diabetes were not adjusted for glucose metabolism status). Finally, all associations, except those describing waist circumference, BMI, and the metabolic syndrome, also included BMI as covariate.

The ISM approach was applied to the cardio-metabolic outcomes that were statistically significantly associated in the regression analyses. The ISM analysis involves a linear 
regression model that includes all types of behavior (sedentary, standing, and stepping) and total waking time. By dropping one type of behavior, e.g. sedentary behavior, the coefficients of another type of behavior, e.g. standing, will represent the estimated effect of replacing $30 \mathrm{~min}$ sedentary time with $30 \mathrm{~min}$ standing. The following estimated replacement effects were modelled: sedentary time replaced by standing and replaced by stepping, and standing replaced by stepping. Since all types of behavior (with all types of intensities) were included in the models, the estimated replacement effects of the behaviors were independent of each other. We used time blocks of $30 \mathrm{~min}$ because a minimum of 30 min moderate to vigorous physical activity per day is required to meet the physical activity guidelines. ${ }^{25}$ The associations were adjusted as described before. In all analyses the assumption of linearity was verified and multi-collinearity was not indicated (variance inflating factors were $<2.5$ ).

In additional analyses, we tested for interaction between all types of behavior (sedentary time, standing, and stepping) and glucose metabolism status. A P-value $<0.10$ for interaction was considered statistically significant. Further, the linear regression analyses were repeated with replacement of office blood pressure with $24 \mathrm{~h}$ average ambulatory blood pressure $(n=1,956)$. All analyses were conducted with IBM SPSS Statistics 22.0 (IBM Corp., Armonk, NY, USA).

\section{Results}

A total of 2,213 participants (51\% men) with an average age of $60.0 \pm 8.1$ years, were included. They provided on average $6.3 \pm 1.2$ valid days of accelerometry data, although most participants (57\%) provided 7 valid days. Average waking time was $15.7 \pm 0.9 \mathrm{~h}$ per day, of which $9.4 \pm 1.7 \mathrm{~h}(60 \%)$ was spent in a sedentary position. The remaining waking hours were spent standing (( $4.3 \pm 1.3 \mathrm{~h}) ; 27 \%$ and stepping $(2.0 \pm 0.7 \mathrm{~h}) ; 13 \%)$. Characteristics of the total study population and according to sex-specific tertiles of sedentary time are presented in Table 6.1. 


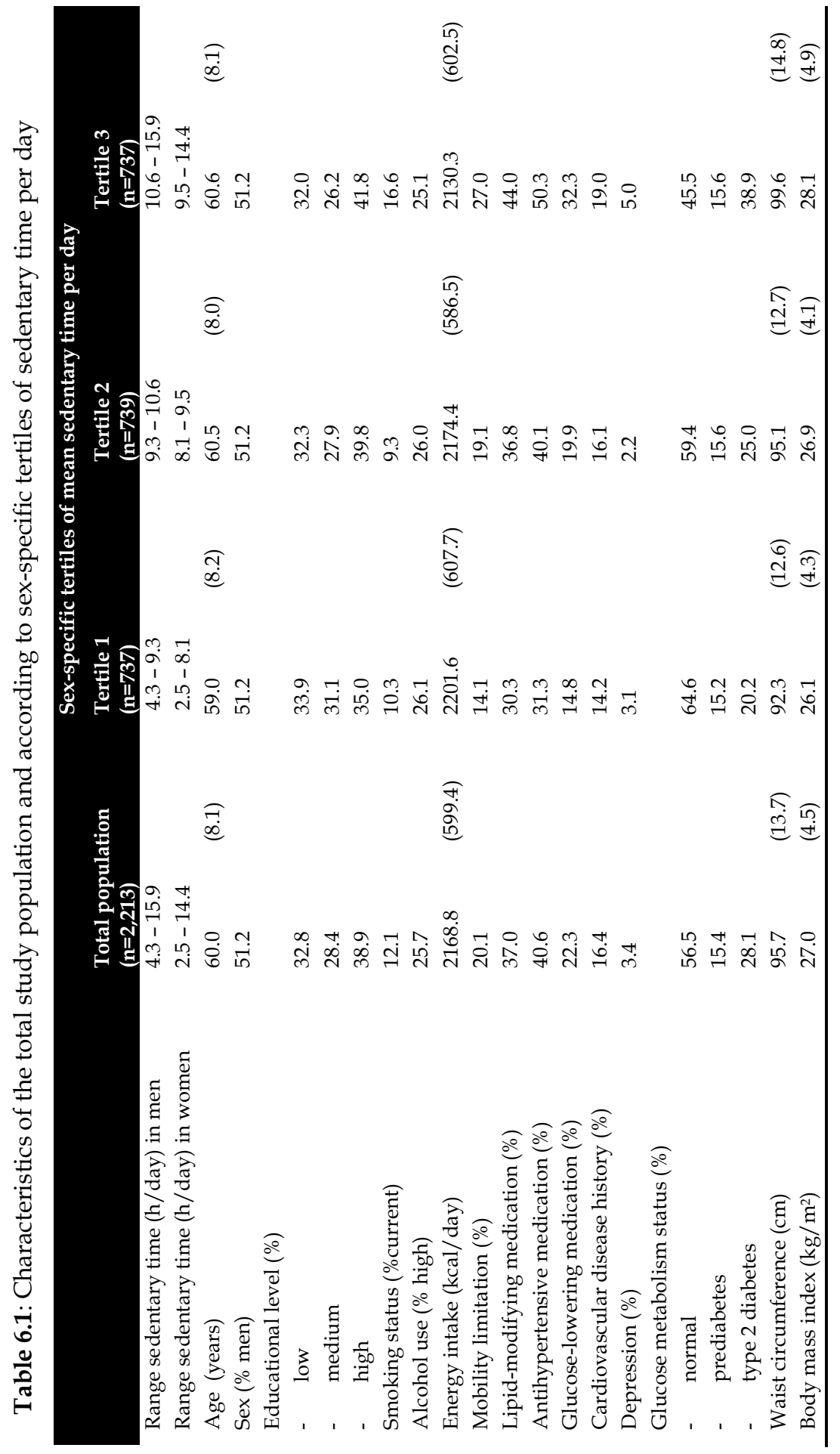




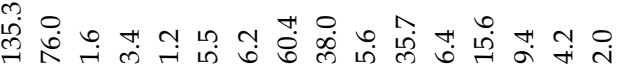


To determine which outcome measures should be studied using the ISM approach, associations between all types of behavior and the cardio-metabolic outcomes were examined separately using regression analyses. Sedentary time was, in the fully adjusted model, statistically significantly associated with a larger waist circumference, a higher BMI, a lower HDL cholesterol level, a higher total-to-HDL cholesterol ratio, and higher triacylglycerol, $2 \mathrm{~h}$ post-load glucose, and a fasting insulin levels. Standing and stepping were associated with a lower waist circumference, a lower BMI, a higher HDL cholesterol level, a lower total-to-HDL cholesterol ratio, and lower triacylglycerol, $2 \mathrm{~h}$ post-load glucose, and fasting insulin levels. No statistically significant associations were observed between any of the types of behavior and blood pressure, fasting glucose levels, and HbA1c. In contrast, all types of behavior were associated with the metabolic syndrome and type 2 diabetes. Data are provided in Table S6.1.

Table 6.2 presents the estimated effects of replacing $30 \mathrm{~min}$ of one type of behavior with another. After adjustment for confounders, a daily replacement of 30 min of sedentary time with $30 \mathrm{~min}$ of standing was associated with a $0.41 \mathrm{~cm}$ more favorable waist circumference $\left(B_{\text {sedentary-standing }}=-0.41 ; 95 \% \mathrm{CI},-0.60 ;-0.21\right)$, while a replacement with stepping was associated with a $1.42 \mathrm{~cm}$ more favorable waist circumference $\left(\mathrm{B}_{\text {sedentary-stepping }}\right.$ $=-1.42(-1.78,-1.06))$. After adjustment for confounders, associations with a more favorable BMI were seen when sedentary time was replaced by stepping $\left(B_{\text {sedentary-stepping }}=\right.$ $-0.48(-0.62 ;-0.35))$, or when standing was replaced by stepping $\left(B_{\text {standing-stepping }}=-0.44\right.$ $(-0.61 ;-0.27))$. Such replacement effects of stepping were also seen for HDL cholesterol $\left(\mathrm{B}_{\text {sedentary-stepping }}=0.04(0.03 ; 0.05) ; \mathrm{B}_{\text {standing-stepping }}=0.04 ;(0.02 ; 0.05)\right)$, and $2 \mathrm{~h}$ post-load glucose levels $\left(\mathrm{B}_{\text {sedentary-stepping }}=0.98(0.97 ; 0.99) ; \mathrm{B}_{\text {standing-stepping }}=0.98(0.97 ; 0.99)\right.$; back transformed from log scale).

For total-to-HDL cholesterol ratio, triacylglycerol and fasting insulin levels, reallocating sedentary time to standing or to stepping resulted in lower total-to-HDL ratios ( $\mathrm{B}_{\text {seden- }}$ tary-standing $=0.99(0.99 ; 1.00) ; B_{\text {sedentary-stepping }}=0.98(0.97 ; 0.99)$; back transformed from log scale), lower triacylglycerol levels $\left(\mathrm{B}_{\text {sedentary-standing }}=0.99(0.98,1.00) ; \mathrm{B}_{\text {sedentary-stepping }}=0.98\right.$ $(0.96 ; 0.99)$; back transformed from log scale), and lower fasting insulin levels ( $B_{\text {sedentary- }}$ standing $=0.99(0.98 ; 1.00) ; B_{\text {sedentary-stepping }}=0.97(0.95 ; 0.99)$; back-transformed from logscale).

Figure 6.1 presents the estimated effects on the metabolic syndrome and type 2 diabetes of replacing $30 \mathrm{~min}$ of one type of behavior with another. After adjustment for confounders associations with the metabolic syndrome were seen when sedentary time was replaced by either standing $\left(\mathrm{OR}_{\text {sedentary-standing }}=0.93(0.89 ; 0.97)\right)$, or stepping $(\mathrm{OR}-$ sedentary-stepping $=0.72(0.66 ; 0.78))$, and when standing was replaced by stepping $\left(\mathrm{OR}_{\text {standing- }}\right.$ stepping $=0.77(0.70 ; 0.86))$. Similar results were seen for type 2 diabetes; after adjustment 
for confounders, associations were seen when sedentary time was replaced by standing $\left(\mathrm{OR}_{\text {sedentary-standing }}=0.94(0.90 ; 0.99)\right)$, or stepping $\left(\mathrm{OR}_{\text {sedentary-stepping }}=0.79(0.72 ; 0.87)\right)$, and when standing was replaced by stepping $\left(\mathrm{OR}_{\text {standing-stepping }}=0.84(0.74 ; 0.95)\right)$.

In additional analyses interaction terms between stepping and glucose metabolism status were statistically significant for waist circumference, BMI, and fasting insulin (all $\mathrm{P}<0.10$ ). Therefore, the replacement analyses were repeated according to glucose metabolism status. Figure 6.2 presents the estimated effects on waist circumference and BMI of replacing $30 \mathrm{~min}$ of sedentary time with equal amounts of standing or stepping, in the NGM, prediabetes and type 2 diabetes groups. Replacing sedentary time with standing as well as stepping was associated with a more favorable waist circumference in all groups, but larger effects were seen in the prediabetes and type 2 diabetes groups, compared to the NGM group. For example, replacing sedentary time with stepping was associated with a $1.05 \mathrm{~cm}$ more favorable waist circumference in the NGM group $\left(\mathrm{B}_{\text {sedentary-stepping }}=-1.05(-1.47 ;-0.63)\right)$, while this was $1.70 \mathrm{~cm}\left(\mathrm{~B}_{\text {sedentary-stepping }}=-1.70(-2.67\right.$; $-0.74))$ and $1.89 \mathrm{~cm}\left(\mathrm{~B}_{\text {sedentary-stepping }}=-1.89(-2.73 ;-1.06)\right)$ in the prediabetes and type 2 diabetes groups, respectively A similar pattern was seen for BMI: replacing sedentary time with stepping was associated with a $0.36 \mathrm{~kg} / \mathrm{m}^{2}$ more favorable BMI in the NGM group $\left(B_{\text {sedentary-stepping }}=-0.39(-0.51 ;-0.20)\right.$, while this was $0.62 \mathrm{~kg} / \mathrm{m}^{2}\left(B_{\text {sedentary-stepping }}=\right.$ $-0.62(-0.98 ;-0.26))$ in the prediabetes group, and $0.62 \mathrm{~kg} / \mathrm{m}^{2}\left(B_{\text {sedentary-stepping }}=-0.62\right.$; $(-0.95 ;-0.30))$ in the type 2 diabetes groups. Although a statistically significant interaction term was found between stepping and glucose metabolism status for fasting insulin, the replacement effects on fasting insulin in the NGM, prediabetes, and type 2 diabetes groups were similar (data not shown).

Further, in additional analyses using $24 \mathrm{~h}$ average ambulatory blood pressure instead of office blood pressure resulted in similar findings; no associations were seen with any type of behavior (data not tabulated). Also, all results remained similar when we excluded participants having $<4$ valid days. 


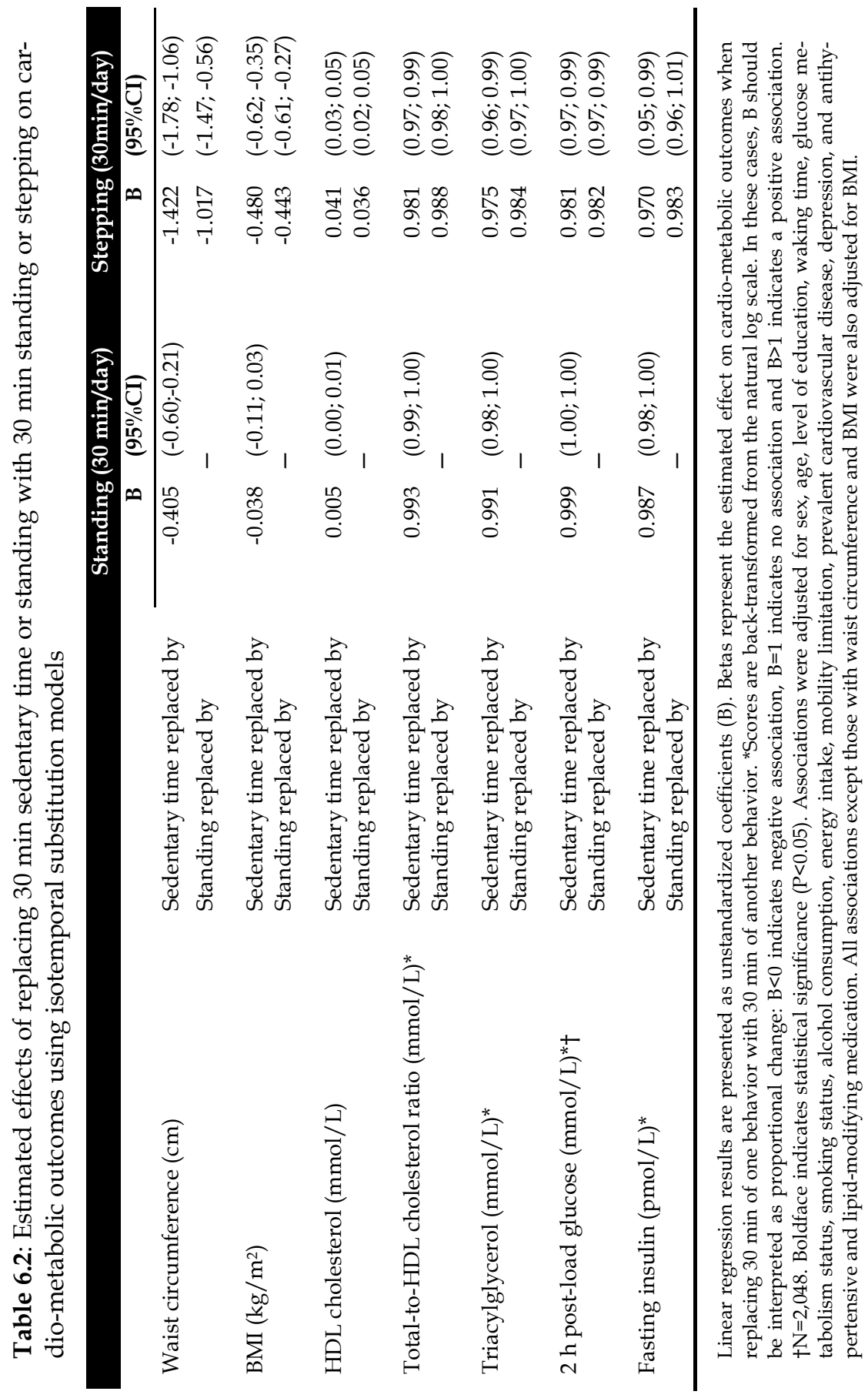




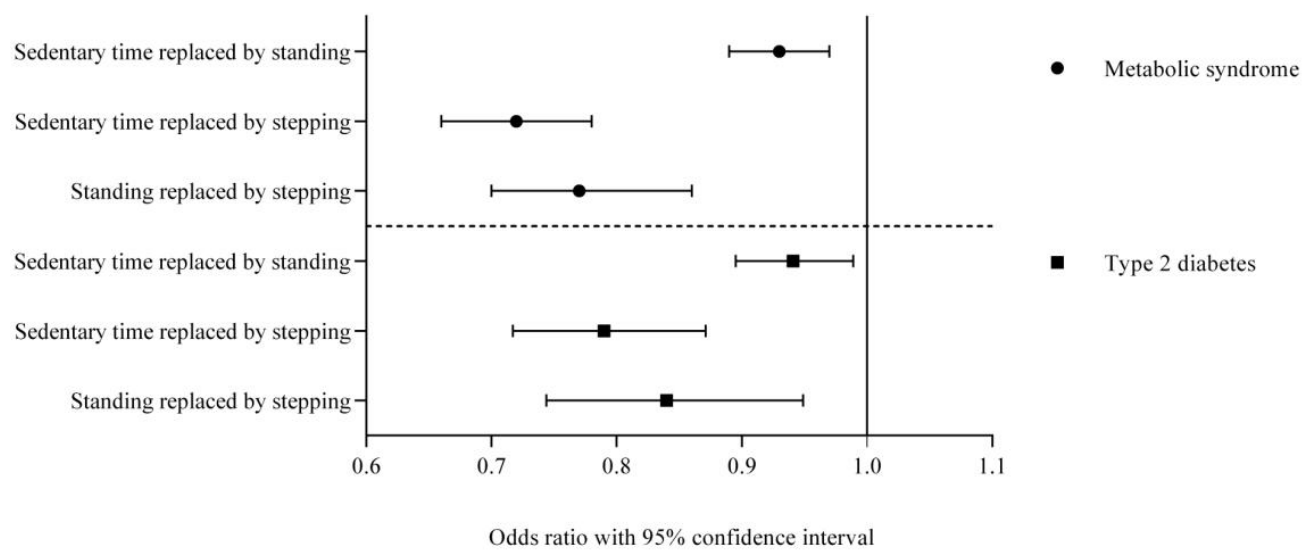

Figure 6.1 Estimated effects of replacing $30 \mathrm{~min}$ of sedentary time or standing with $30 \mathrm{~min}$ of standing or stepping on the metabolic syndrome and type 2 diabetes, using isotemporal substitution models. Odds ratios represent the odds for metabolic syndrome or type 2 diabetes when replacing $30 \mathrm{~min}$ of one behavior with $30 \mathrm{~min}$ of another behavior. Associations were adjusted for sex, age, level of education, waking time, smoking status, alcohol consumption, energy intake, mobility limitation, prevalent cardiovascular disease, and depression. Type 2 diabetes was also adjusted for body mass index.

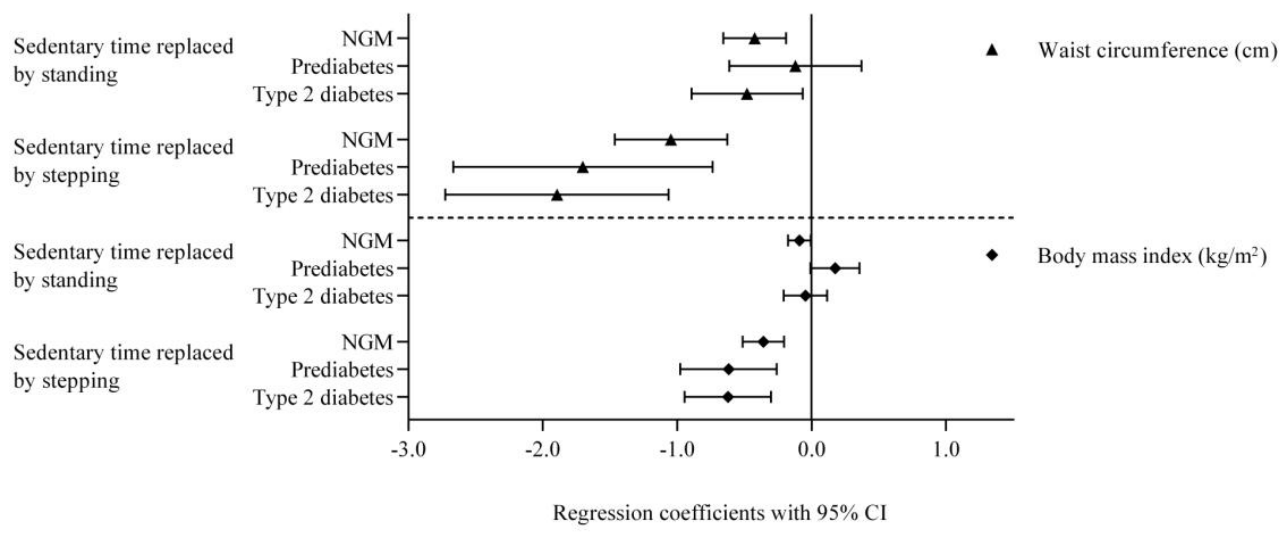

Figure 6.2 Estimated effects of replacing $30 \mathrm{~min}$ of sedentary time with $30 \mathrm{~min}$ of standing or stepping on waist circumference and body mass index, according to glucose metabolism status, using isotemporal substitution models. Regression coefficients represent the estimated effect on waist circumference and body mass index when replacing $30 \mathrm{~min}$ of one behavior with $30 \mathrm{~min}$ of another behavior. NGM, normal glucose metabolism. Associations were adjusted for sex, age, level of education, waking time, smoking status, alcohol consumption, energy intake, mobility limitation, prevalent cardiovascular disease, depression, and antihypertensive and lipid-modifying medication. 


\section{Discussion}

In this study, we used the ISM approach to estimate the effects of a theoretical reallocation of sedentary behavior (sitting or lying) to standing or stepping with cardiometabolic outcomes, the metabolic syndrome, and type 2 diabetes. The results show that, after adjustment for confounders, reallocating $30 \mathrm{~min}$ of sedentary time per day to standing or stepping, was significantly associated with a 7 to $28 \%$ lower odds for the metabolic syndrome, and a 6 to $21 \%$ lower odds for type 2 diabetes. Further, replacing sedentary behavior with non-sedentary behavior was beneficially associated with several metabolic outcomes. The largest estimated effects were seen when sedentary time was replaced by stepping: replacing $30 \mathrm{~min}$ sedentary time per day by stepping resulted in a $1.4 \mathrm{~cm}$ more favorable waist circumference, a $0.5 \mathrm{~kg} / \mathrm{m}^{2}$ more favorable BMI, and improved levels of cholesterol (both HDL and total-to-HDL ratio), triacylglycerol, and $2 \mathrm{~h}$ post-load glucose. Also, the replacement of sedentary behavior with standing was associated with a more favorable waist circumference, a lower total-to-HDL cholesterol ratio, a lower triacylglycerol level, and a lower insulin level. In addition, reducing standing by replacing it with stepping was associated with more favorable waist circumference and BMI, improved HDL cholesterol and $2 \mathrm{~h}$ post-load glucose levels, and lower odds for the metabolic syndrome and type 2 diabetes. Finally, when estimating the effects of replacing sedentary time with standing or stepping in groups according to glucose metabolism status, larger replacement effects were seen in participants with prediabetes and type 2 diabetes compared to participants with NGM for both waist circumference and BMI. However, this was likely due to the larger measures of waist circumference and BMI in the prediabetes and type 2 diabetes groups rather than glucose metabolism per se. Taken together, the results suggest that reallocating sedentary behavior, even in relatively small amounts of $30 \mathrm{~min} /$ day, may have favorable effects on metabolic outcomes, the metabolic syndrome, and type 2 diabetes. Interestingly, these favorable results were not only seen when sedentary time was replaced by stepping (e.g. physical activity), but also when it was replaced by standing. Also, as stronger associations for waist circumference and BMI were seen in participants with prediabetes and type 2 diabetes, reallocation of only $30 \mathrm{~min}$ of sedentary time per day to standing or stepping may be clinically relevant. Thus, our results provide new insight in the potential effects of reallocating sedentary time, which may be used for health guidelines and may also give direction to experimental or intervention studies on the amounts of reallocation time that should be examined. For these reasons, our findings are relevant for the general population, for those who cannot participate in high-intensity activities, and for those who cannot meet the physical activity guidelines. 
Our results are in line with findings of previous studies using the ISM approach. Most of these studies reported associations of reallocating sedentary time to LPA or MVPA with reductions in waist circumference, ${ }^{10-12,14,15}$ and BMI.10-13 Less consistent results were seen for other metabolic outcomes including levels of cholesterol, triacylglycerol, and glucose. In a few studies including ours, ${ }^{12,15}$ statistically significant associations were seen with HDL cholesterol when replacing sedentary time with more active types of behavior, while other studies showed no associations, ${ }^{10}$ or only associations with MVPA.13,14 Similar inconsistencies were seen for triacylglycerol,12-15 and glucose levels. ${ }^{12,14,16}$ These inconsistencies could be due to the study population which varied in age (36 to 80 years), and in metabolic profile (healthy participants, participants at increased risk for type 2 diabetes, participants with type 2 diabetes). In addition, the replacement time varied between studies, as some studies used blocks of $10 \mathrm{~min}, 13,15$ while we and others used 30 min blocks, ${ }^{11,14,16}$ and even 2 h blocks were used. ${ }^{12}$ Further, most studies used a waist- or hip-worn accelerometer which cannot distinguish between postures. Consequently, estimations of the amount of sedentary time in these studies were less accurate than the estimates in our study, as we used a posture-based accelerometer. ${ }^{17-19}$ In only one other study a posture-based accelerometer was used and the reported results were similar to ours, although in that study an allocation time of 2 hours was used. ${ }^{12}$

Possible pathophysiological mechanisms that may explain our findings have not yet been studied extensively. However, animal studies have described plausible mechanisms that may underlie the detrimental health effects of large amounts of sedentary time, including reductions in lipoprotein lipase (LPL) and lipid phosphate phosphatase-1 (LPP1), due to muscle inactivity. ${ }^{26,27}$ Participation in non-sedentary behavior may be sufficient to counterbalance these processes. It has been shown that reducing inactivity by low intensity activities, such as walking and standing, was effective in improving insulin level and plasma lipids, which supports our hypothesis. ${ }^{28}$ Additionally, previous experimental and epidemiological studies have demonstrated that breaking up sedentary time with non-sedentary time had beneficial effects on metabolic outcomes, which supports also this hypothesis. ${ }^{29}$ Nevertheless, physiological studies are needed to further examine this.

The use of posture-based accelerometer was a key strength of this study, as well as the large study population consisting of adults with NGM, prediabetes, and type 2 diabetes. Another strength of this study was adjustment for a series of relevant confounders, including total energy intake. However, this could have resulted in overadjustment bias as an increase in standing or stepping could result in a higher energy intake. 
A few limitations should also be mentioned. Due to the cross-sectional nature of the study, causal relationships could not be determined and reverse causality could not be excluded. Moreover, the replacement effects resulting from the ISM approach were estimated rather than based on actual behavioral reallocation. Although this method can provide valuable insights in the potential effects on health, experimental and intervention studies are warranted to examine the actual behavioral reallocation effects on both short- and long-term. Further, as our accelerometry data were posture-based, we used total stepping time as a measure for physical activity, but different intensity levels were not determined. In addition, stepping time was not classified into higher and lower intensity stepping, because the associations of lower intensity stepping with the outcome measures were nonlinear. Therefore, future studies ideally should combine posture-based data for accurate assessment of sedentary behavior with acceleration data for determining physical activity intensity levels. In addition, due to missing data, we had to exclude 1,200 participants. However, the excluded participants did not differ from our study sample with regard to demographic factors, accelerometry variables, and outcomes measures (data not shown). Finally, our study population consisted of a relatively healthy population including individuals with well-controlled type 2 diabetes; therefore, the results might not be representative for the general population of adults aged 40-75 years.

To conclude, our study demonstrated that a theoretical replacement of sedentary time with standing or stepping was associated with a decreased likelihood for the metabolic syndrome and type 2 diabetes, more favorable waist circumference and BMI, and improved levels of HDL cholesterol, triacylglycerol, glucose and insulin. In addition, replacing standing with stepping was associated with an improved waist circumference, BMI, HDL cholesterol and glucose level, the metabolic syndrome, and type 2 diabetes. Further, the theoretical replacement effects for both waist circumference and BMI were larger in participants with prediabetes and type 2 diabetes. As reallocating of even small amounts of sedentary behavior to any type of non-sedentary behavior seems to be beneficial for health, consideration should be given to developing health guidelines that include recommendations for the daily reallocation of sedentary time, although longitudinal and intervention studies on actual behavioral reallocation effects are needed to confirm our results. 


\section{References}

1. Sedentary Behaviour Research Network. (2102). Letter to the editor: standardized use of the terms "sedentary" and "sedentary behaviours". Appl. Physiol. Nutr. Metab;37:540-542.

2. Brocklebank LA, Falconer CL, Page AS, Perry R, Cooper AR. Accelerometer-measured sedentary time and cardiometabolic biomarkers: A systematic review. Prev. Med. 2015;76:92-102.

3. Edwardson CL, Gorely T, Davies MJ, Gray LJ, Khunti K, Wilmot EG, Yates T, Biddle SJ. Association of sedentary behaviour with metabolic syndrome: a meta-analysis. PLoS ONE 2012;7(4):e34916.

4. Biswas A, Oh PI, Faulkner GE, Bajaj RR, Silver MA, Mitchell MS, Alter DA. Sedentary time and its association with risk for disease incidence, mortality, and hospitalization in adults: a systematic review and meta-analysis. Ann. Intern. Med. 2015;162(2):123-132.

5. Wilmot EG, Edwardson CL, Achana FA, Davies MJ, Gorely T, Gray LJ, Khunti K, Yates T, Biddle SJ. Sedentary time in adults and the association with diabetes, cardiovascular disease and death: systematic review and meta-analysis. Diabetologia 2012;55(11):2895-2905.

6. Canadian. Society of Exercise Physiology. Canadian Physical Activity and Sedentary Behaviour Guidelines. 2012 [cited September 2016]. Available from: www.csep.ca/guidelines.

7. Department. of Health Physical Activity Health Improvement and Protection. Start Active , Stay Active. 2011 [cited September 2016]. Available from: https://www.gov.uk/government/publications/start-active-stay-active-a-report-on-physicalactivity-from-the-four-home-countries-chief-medical-officers.

8. Australian. Government Department of Health. Australia's Physical Activity and Sedentary Behaviour Guidelines For Adults (18-64 years). 2014 [cited September 2016]. Available from: http://www.health.gov.au/internet/main/publishing.nsf/Content/phy-activity.

9. Mekary RA, Willett WC, Hu FB, Ding EL. Isotemporal substitution paradigm for physical activity epidemiology and weight change. Am. J. Epidemiol. 2009:kwp163.

10. Falconer CL, Page AS, Andrews RC, Cooper AR. The potential impact of displacing sedentary time in adults with type 2 diabetes. Med. Sci. Sports Exerc. 2015;47(10):2070.

11. Healy GN, Winkler EA, Brakenridge CL, Reeves MM, Eakin EG. Accelerometer-derived sedentary and physical activity time in overweight/obese adults with type 2 diabetes: cross-sectional associations with cardiometabolic biomarkers. PLoS ONE 2015;10(3):e0119140.

12. Healy GN, Winkler EA, Owen N, Anuradha S, Dunstan DW. Replacing sitting time with standing or stepping: associations with cardio-metabolic risk biomarkers. Eur. Heart J. 2015:ehv308.

13. Hamer M, Stamatakis E, Steptoe A. Effects of substituting sedentary time with physical activity on metabolic risk. Med. Sci. Sports Exerc. 2014;46(10):1946-1950.

14. Buman MP, Winkler EA, Kurka JM, Hekler EB, Baldwin CM, Owen N, Ainsworth BE, Healy GN, Gardiner PA. Reallocating time to sleep, sedentary behaviors, or active behaviors: associations with cardiovascular disease risk biomarkers, NHANES 2005-2006. Am. J. Epidemiol. 2013:kwt292.

15. Ekblom-Bak E, Ekblom Ö, Bergström G, Börjesson M. Isotemporal substitution of sedentary time by physical activity of different intensities and bout lengths, and its associations with metabolic risk. Eur J Prev Cardiol 2016;23(9):967-974.

16. Yates T, Henson J, Edwardson C, Dunstan D, Bodicoat DH, Khunti K, Davies MJ. Objectively measured sedentary time and associations with insulin sensitivity: Importance of reallocating sedentary time to physical activity. Prev. Med. 2015;76:79-83.

17. Kozey-Keadle S, Libertine A, Lyden K, Staudenmayer J, Freedson PS. Validation of wearable monitors for assessing sedentary behavior. Med. Sci. Sports Exerc. 2011;43(8):1561-7.

18. Lyden K, Kozey-Keadle SL, Staudenmayer JW, Freedson PS. Validity of two wearable monitors to estimate breaks from sedentary time. Med. Sci. Sports Exerc. 2012;44(11):2243.

19. Godfrey A, Culhane K, Lyons G. Comparison of the performance of the activPAL ${ }^{\mathrm{TM}}$ Professional physical activity logger to a discrete accelerometer-based activity monitor. Med. Eng. Phys. 2007;29(8):930-934.

20. Schram MT, Sep SJ, van der Kallen CJ, Dagnelie PC, Koster A, Schaper N, Henry RM, Stehouwer $\mathrm{CD}$. The Maastricht Study: an extensive phenotyping study on determinants of type 2 diabetes, its complications and its comorbidities. Eur. J. Epidemiol. 2014;29(6):439-51. 
21. Alberti K, Eckel RH, Grundy SM, Zimmet PZ, Cleeman JI, Donato KA, Fruchart J-C, James WPT, Loria CM, Smith SC. Harmonizing the metabolic syndrome a joint interim statement of the international diabetes federation task force on epidemiology and prevention; national heart, lung, and blood institute; American heart association; world heart federation; international atherosclerosis society; and international association for the study of obesity. Circulation 2009;120(16):1640-1645.

22. World Health Organization. Definition and Diagnosis of Diabetes Mellitus and Intermediate Hyperglycemia. 2006.

23. van der Berg JD, Willems PJ, van der Velde JHPM, Savelberg HHCM, Schaper NC, Schram MT, Sep SJ, Dagnelie PC, Bosma H, Stehouwer CDA. Identifying waking time in 24-h accelerometry data in adults using an automated algorithm. J. Sports Sci. 2016;34(19):1867-73.

24. Sheehan D, Lecrubier Y, Sheehan KH, Sheehan K, Amorim P, Janavs J, Weiller E, Hergueta T, Baker R, Dunbar G. Diagnostic Psychiatric Interview for DSM-IV and ICD-10. J. Clin. Psychiatry 1998;59:22-33.

25. Hildebrandt. H, Ooijendijk M, Hopman M. Trend report Physical Activity and Health 2000/2014. ("Trendrapport Bewegen en Gezondheid 2000/2014"). 2010. p.16. 2010.

26. Hamilton MT, Hamilton DG, Zderic TW. Role of low energy expenditure and sitting in obesity, metabolic syndrome, type 2 diabetes, and cardiovascular disease. Diabetes 2007;56(11):2655-2667.

27. Hamilton MT, Hamilton DG, Zderic TW. Sedentary behavior as a mediator of type 2 diabetes. Diabetes and Physical Activity. Vol. 60 Karger Publishers, 2014;11-26.

28. Duvivier BM, Schaper NC, Bremers MA, van Crombrugge G, Menheere PP, Kars M, Savelberg HH. Minimal Intensity Physical Activity (Standing and Walking) of Longer Duration Improves Insulin Action and Plasma Lipids More than Shorter Periods of Moderate to Vigorous Exercise (Cycling) in Sedentary Subjects When Energy Expenditure Is Comparable. PLoS ONE 2013;8(2):e55542.

29. Benatti FB, Ried-Larsen M. The effects of breaking up prolonged sitting time. Med. Sci. Sports Exerc. 2015. 
Supplement to chapter 6 


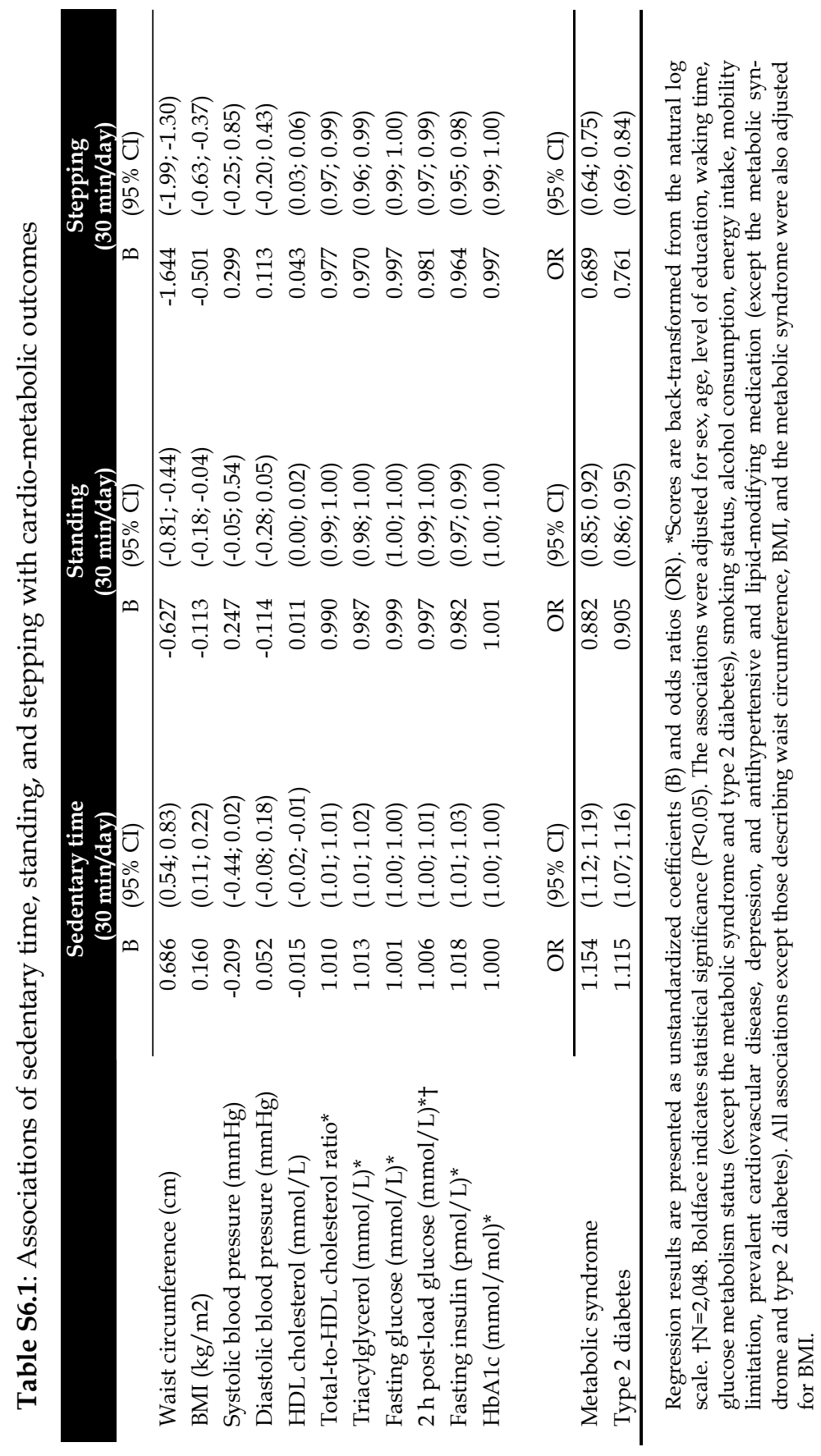




\section{Chapter 7}

Summary and general discussion 
This chapter summarizes, after a brief introduction, the main findings of this thesis. In addition, the (biological) plausibility of our findings is discussed and several methodological considerations are addressed. Finally, implications and directions for future research are provided.

As described in chapter 1, the lack of physical activity is one of the key modifiable risk factors for non-communicable diseases (NCDs), such as cardiovascular disease and type 2 diabetes mellitus (T2DM). ${ }^{1-4}$ Physical activity research has typically focused on moderate to vigorous physical activity (MVPA), activities $\geq 3.0$ metabolic equivalents (METs)), and has recognized MVPA as an important factor to improve cardio-metabolic health, physical function and cardiorespiratory fitness (CRF).5,6 However, MVPA comprises only a relatively small part of all daily activities and most people spend the majority of the day in sedentary behaviour; any waking behaviour, characterized by a low energy expenditure ( $\leq 1.5 \mathrm{METs})$, while being in a sitting or reclining position. ${ }^{7-11}$ Sedentary behaviour has been identified as a risk factor for adverse health outcomes, regardless of the amount of MVPA. 10,12,13 However, it is largely unknown if a large amount of daily sedentary time is associated with lower CRF or lower physical function. In addition, if sedentary behaviour negatively affects cardio-metabolic health regardless of CRF is uncertain. Further, a general limitation of physical activity and sedentary behaviour research to date is that the amount and intensity of daily activities is often assessed using self-reported measures, which have limited reliability and validity. ${ }^{14,15}$

Therefore, the overall aim of this thesis was to improve the insight into sedentary behaviour, physical activity, and CRF and their interrelationship, as risk factors for cardio-metabolic health. First, we examined the association of sedentary behaviour and physical activity with physical function and CRF. Second we examined the independent and combined associations of sedentary time, higher intensity physical activity (corresponding approximately with MVPA), and CRF with cardio-metabolic health. For this we used data of two observational studies: The Maastricht Study and The National Health and Nutrition Examination Survey (NHANES). In both studies daily activities were measured by accelerometry.

\section{Summary of main findings}

In chapter 2, the associations of objectively measured sedentary time, patterns of sedentary behaviour, and (higher intensity) physical activity with physical function were examined in adults aged 40-75 years (The Maastricht Study). Measures of physical function included walking speed and muscle strength. The amount of sedentary time and patterns of sedentary behaviour appeared to be only weakly associated with lower physical function, independent of higher intensity physical activity. In contrast, engag- 
ing regularly in physical activity, in particular higher intensity physical activity, was associated with better physical function.

In chapter 3, the associations of objectively measured sedentary time, patterns of sedentary behaviour, and lower and higher intensity physical activity with CRF were examined in adults aged 40-75 years (The Maastricht Study). Larger amount of sedentary time was associated with lower CRF, independent of higher intensity physical activity. Patterns of sedentary behaviour were not associated with CRF. Results from the isotemporal substitution analyses revealed that replacing sedentary time with either lower or higher intensity physical activity was associated with higher CRF. The strongest association was observed with higher intensity physical activity. Replacing sedentary time with standing was not associated with higher CRF.

In chapter 4, the mutually independent associations of sedentary time, higher intensity physical activity and CRF with cardio-metabolic health were examined in adults aged 18-49 years (NHANES). Results showed that more time engaged in higher intensity physical activity and higher CRF were independently associated with an advantageous cardio-metabolic risk profile: lower waist circumference, lower level of blood lipids, and higher level of HDL-cholesterol. On the other hand, sedentary time was not associated with cardio-metabolic risk independent of the amount of higher intensity physical activity and CRF.

Similar associations were examined in chapter 5, but this time in adults aged 40-75 years (The Maastricht Study). In this population low sedentary time, more time engaged in higher intensity physical activity and high CRF were each associated with several markers of cardio-metabolic health and lower risk for the metabolic syndrome and T2DM independent of each other. Strength of the association of CRF with cardiometabolic health appeared to be largest. Subsequently we analysed different combinations of CRF - higher intensity physical activity and CRF - sedentary time. In these analyses, CRF was the most dominant factor. People with low CRF, either in combination with frequent higher intensity physical activity or low sedentary time had a larger risk for a detrimental cardio-metabolic risk profile than people with a high CRF but low level of higher intensity physical activity or largest amount of sedentary time.

In chapter 6, we examined the associations with cardio-metabolic health, the metabolic syndrome and T2DM of theoretical replacement of sedentary time with standing or stepping in adults aged 40-75 years (The Maastricht Study). Replacing 30 minutes of sedentary time with both standing as well as stepping was beneficially associated with several markers of cardio-metabolic health (e.g. lower waist circumference and lower blood lipids) and with a lower odds for the metabolic syndrome and T2DM.

Figure 7.1 illustrate sthe results of the investigated associations. 


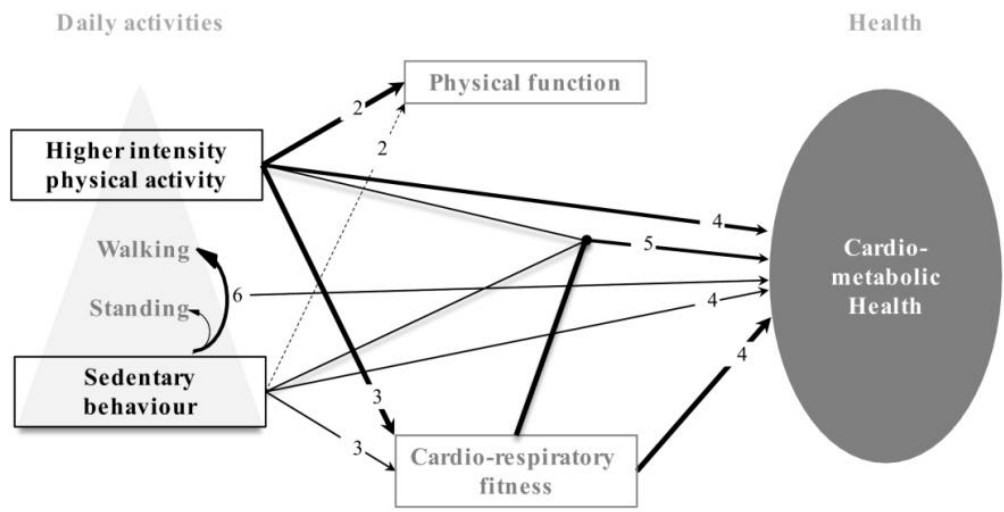

Figure 7.1: Schematic representation of the associations studied in this thesis. Numbers represent corresponding chapters. Thickness of the line indicates strength of the association, dashed line indicates weak association.

\section{Explanation of findings}

\section{Physical activity and cardiorespiratory fitness}

In this thesis, physical activity, in particular higher intensity physical activity (or MVPA), was associated with better cardio-metabolic health, and with higher CRF and physical function. These observations add to the vast amount of studies presenting health benefits of physical activity and support the relevance of physical activity guidelines. The mechanisms through which physical activity improves cardio-metabolic risk and improves physical function have been investigated thoroughly in many studies. These mechanisms are intertwined and include improved body composition, adaptations in skeletal muscle tissue, improved delivery of oxygen and nutrients to skeletal muscle tissue and stimulation of protective mechanisms. ${ }^{16-21}$ Changes in body composition include reduced visceral adiposity and increased fat free mass. Adaptations in skeletal muscle tissue include an increase in oxidative capacity, improving glucose and lipid metabolism. Greater oxidative capacity is partly caused by an increase in capillary density, improving delivery of oxygen and nutrients to muscle tissue and to improvements in the number and the function of mitochondria. ${ }^{22}$ Delivery of oxygen and nutrients to skeletal muscle tissue is further enhanced by improved cardiovascular and respiratory function (e.g. improved coronary blood flow and augmented cardiac output), improved endothelial function (as a result of shear stress), and an enhanced transport and uptake of lipids (e.g. increased fatty-acid-binding proteins (FABPs)) and glucose 
(e.g. through GLUT-4 protein translocation). Protective mechanisms involve a higher level of anti-oxidants and DNA repair mechanisms, improving cell longevity. Altogether, these mechanisms contribute to a reduced blood pressure, prothrombotic coagulation profile, reduced triglyceride levels, increased HDL-cholesterol levels and decreased LDL-to-HDL ratios, improved insulin sensitivity, lower glucose levels, lower levels of inflammatory markers and reactive oxygen species. ${ }^{16-21}$

The mechanisms through which physical activity improves cardio-metabolic risk are mainly studied in the light of chronic adaptations to physical activity and depend on the intensity and duration of these activities. As these adaptations also lead to increased CRF, explanations of protective effects of CRF and physical activity are considered to be similar. ${ }^{17,21,23}$ Further, next to above-mentioned long-term adaptations, MVPA results in short-term improvements in blood pressure, blood lipids, and glucose metabolism. ${ }^{24}$ These short-term improvements indicate that even when not performed on a regular base (and consequently not improving or maintaining CRF) MVPA can have health benefits.

Our understanding on the exact dose-response relation of physical activity is limited. Most physical activity guideline state that people should engage at least 150 minutes per week in MVPA, which unmistakably has health benefits. ${ }^{18}$ At the same time, slowly running for only 5-10 minutes per day has been linked with a reduced risk for mortality from all causes and cardiovascular disease..$^{25}$ On the other hand, increasing the amount of MVPA above 150 minutes per week will lead to greater risk reductions for cardiovascular disease and mortality. ${ }^{4}$ Further, some guidelines state that MVPA should be performed in bouts of at least 10 minutes. However, the requirement of this minimum bout duration has been debated over the last years. ${ }^{26}$ Further, in order to increase CRF a minimum bout duration of vigorous physical of 20 minutes is advised. ${ }^{27,28}$ However, interval training with high intensity bouts of short duration $(<5$ minutes) appear even better than continuous bouts of moderate intensity (while performing similar total work) for improving CRF or cardio-metabolic rsik.29,30 Overall, additional research is required to determine optimal dose-response relations of physical activity; at what frequency, intensity, and duration is physical activity most effective in improving risk factors for cardio-metabolic health?

\section{Sedentary behaviour}

In this thesis, we have shown that a larger amount of sedentary time, assessed using a posture-based accelerometer, was deleteriously associated with markers of cardiometabolic health, and with greater odds for the metabolic syndrome and T2DM in adults aged 40-75 years. Of note, these associations were observed after adjustment for higher intensity physical activity and CRF, suggesting that the amount of sedentary is a 
distinct risk factor for diseases, as reported by others. ${ }^{31-36}$ However, the mechanisms and biological pathways of how prolonged sedentary time affects health are still largely unknown. As the amount of sedentary time and the pattern in which this was accumulated showed only modest associations with CRF and physical function (chapters 2 and $3)$, these factors are unlikely to explain the detrimental health effects of prolonged sedentary time.

An explanation how prolonged sitting affects cardio-metabolic health could be a positive energy balance: a large amount of time spent in sedentary time may results in a positive energy balance, leading to overweight or obesity. Indeed Levine et al. have described the importance of everyday light intensity physical activities, or which they called non-exercise activity thermogenesis (NEAT), as important factor in the aetiology of obesity. ${ }^{37,38}$ NEAT contributes more to daily energy expenditure than exercise (purposeful MVPA) and varies greatly between individuals. The explanation that the lack of overall physical activity is the underlying problem has been suggested in some studies. ${ }^{39,40}$ For example, no associations were found between the amount of sedentary time and cardio-metabolic risk, when adjusted for or total physical activity, ${ }^{39}$ or with insulin sensitivity, after adjustment for total energy expenditure. ${ }^{40}$ Further, if daily energy expenditure is lower than energy intake (due to sedentary behaviour and/or low NEAT), this would lead to increased adiposity. Consequently, the detrimental health effects of sedentary behaviour could (partly) be mediated by adiposity. Indeed, our results from chapter 5 and 6 and results from others show an association between large amount of sedentary time and higher adiposity. On the other hand, after adjustment for a measure of adiposity, we still observed detrimental associations of a larger amount of sedentary time with lower HDL-cholesterol and higher triglycerides levels (chapter 5 and 6). These data suggest that adiposity is not the sole explanation and different mechanisms are involved that explain how prolonged sitting affects cardio-metabolic health. A first indication that these distinct mechanisms exist, was derived from bed rest studies.

In one study, researchers analysed skeletal muscle gene expression, before bed rest, immediately after bed rest, and after 4 weeks of physical activity training. After 9 days of bed rest, the expression of 4,500 mRNAs was altered. After 4 weeks of exercise (MVPA), over $20 \%$ of these mRNAs were not normalized, leading the researchers to suggest that the biological pathways of physical inactivity are not merely the reverse of physical activity. ${ }^{13,41}$ Another proposed mechanism from bed rest studies is a change in vascular structure and endothelial function in resistance vessels (which include small arteries, arterioles, and metarterioles). These changes are thought to occur mainly via vasoconstrictor pathways, in contrast to physical activity, which acts through vasodilator pathways involving nitric oxide. ${ }^{42,43}$ However, it is important to acknowledge that 
bed rest models induce abrupt and extreme physical inactivity and are therefore not representative of human sedentary behaviour. Nevertheless, these types of studies have provided preliminary insights that distinct mechanisms of sedentary behaviour are probable.

Additional insight in mechanisms of how excess sedentary time may affect health comes from animal studies. In rats, suppressed activity of lipoprotein lipase (LPL) in muscle tissue was observed, due to the absence of contractile activity of skeletal muscles. LPL is a key-enzyme in the process of lipid metabolism and a reduced activity of this enzyme may explain the effects of prolonged sedentary time on lipid metabolism. ${ }^{44}$ Reduced activity of AMP-activated protein kinase, which plays a key role as a regulator of cellular energy homeostasis, has also been suggested as underlying mechanism. ${ }^{45}$

Experimental studies in humans have also suggested that specific pathophysiological responses occur when seated and skeletal muscle groups are not engaged. In experimental cross-over trials, participants followed a treatment of several hours of uninterrupted sitting and a treatment in which they interrupted sitting with short breaks of standing and walking. Interrupting prolonged sitting with regular short bouts of light intensity physical activity improved glucose metabolism. ${ }^{46-48}$ Similar studies were performed in a more free-living setting over a period of 4 days. Under similar energy expenditure, interrupting sedentary time with regular short breaks of light intensity activity was even more positively associated with some cardio-metabolic risk markers than one daily bout of MVPA after prolonged sedentary time. ${ }^{49,50}$ These observations suggest that the negative effect of sedentary time is not just the result of low energy expenditure. However, the exact mechanisms and at what dose or frequency sedentary behaviour starts to negatively affect health should be examined in future studies.

\section{Sedentary behaviour and physical activity vs. cardiorespiratory fitness}

Studies that compared the strength of the associations of MVPA and CRF on cardiometabolic health and risk reduction for mortality, reported a stronger effect for CRF.51-53 The stronger effect for CRF was partly ascribed to a greater accuracy of the measurement of CRF compared with MVPA, which was based on self-report. Nevertheless, when we measured MVPA objectively, as reported in chapter 5, CRF was more strongly associated than MVPA with the risk of having the metabolic syndrome and T2DM, in line with another cross-sectional study. ${ }^{54} \mathrm{~A}$ possible explanation for the stronger association of CRF, is that physiological effects of physical activity are transitory and CRF is determined over a longer period of time. ${ }^{55}$ Thus, in order to retain the beneficial effects of physical activity, it is important to engage in MVPA regularly (use it or lose it) and CRF is a reflection of this. In addition, next to the amount and intensity of MVPA, CRF is determined by genetic and external factors. ${ }^{56-58}$ Hypothetically, a genetic 
predisposition towards high CRF coincides with a predisposition for better cardiometabolic health. Further, it seems reasonable to assume that an attribute as CRF, which by its definition depends on the capacity of the cardiovascular (and respiratory) systems, is more closely related to cardio-metabolic health than MVPA, as cardiometabolic health is also linked with the function of the cardiovascular system. In addition, since sedentary behaviour has been associated with cardio-metabolic health, perhaps all daily activities combined (MVPA, light intensity physical activity, and sedentary behaviour) can be as good as a predictor as CRF for future adverse health.

\section{Methodological considerations}

The findings from the studies in this thesis require some methodological considerations. In this section, factors that may affect the internal and external validity of these studies are discussed. Internal validity refers to the ability to attribute the study results to the variable of interest, and not to other factors. That is, does exposure $X$ (e.g. sedentary time) indeed cause outcome Y (e.g. T2DM)? External validity reflects the degree to which results of a study may apply, to people who did not participate in the study. In other words, can the results of our studies be generalized to other situations or populations? ${ }^{59}$

The internal validity of a study can be affected by systematic errors. ${ }^{60}$ Systematic error, or bias, refers to errors leading to results or conclusions that systematically deviate from truth. These errors may arise from the design of a study, or from the collection, analysis, interpretation, or reporting of data. ${ }^{59}$ Although we attempted to minimise bias in the present thesis, it cannot be dismissed. A number of factors may bias the observed associations in this thesis. These factors are partly inherent to the study design and include selection bias, information bias, confounding, and overadjustment.

\section{Study design}

All analyses in The Maastricht Study as well as in NHANES were of observational nature, with cross-sectional data analyses. Therefore, causality could not be determined. As both the independent variables and the outcomes were measured at the same point in time, reverse causation cannot be excluded. ${ }^{61}$ For instance in chapter 3, it is not unlikely that people with a high level of CRF engage more often in physical activities because they are fit, and people with low CRF engage more in sedentary behaviour because they tire more quickly. However, by adjusting for potential confounders, such as (history of) CVD or the presence of mobility limitations, we attempted to reduce the risk for reverse causation. Analyses of longitudinal data, which should become available for The Maastricht Study in the future, are needed to establish causality. 


\section{Selection bias}

The result of selection bias is that the association between exposure and outcome in the study population differs from the association of those also eligible for the study. ${ }^{62} \mathrm{In}$ other words, the 'true' association was not observed. This type of bias can be due to the procedures to include participants in the study or to factors that influence participation in the study.

For The Maastricht Study, participants were recruited through mass media campaigns and from the municipal registries and the regional Diabetes Patient Registry via mailings. ${ }^{63}$ This type of recruiting may have introduced a type of selection bias: selective non-response bias. This type of bias refers to the concept that individuals who choose to participate in an observational study, such as The Maastricht Study, are different from those who choose not to participate. ${ }^{64}$ A relatively high education level of The Maastricht Study participants may indicate selective non-response bias. Further, the participants with T2DM were relatively well treated: they had relatively low $\mathrm{HbA1C}$ levels, and few reported diabetic complications. Consequently, all associations observed in The Maastricht Study may be an underestimation of the actual associations, as the study population is, to some extent, healthier than the general population. For instance, it could be possible that the participants with T2DM included in The Maastricht Study have a higher CRF, and engage less in sedentary behaviour than those with T2DM and not included in The Maastricht Study, resulting in weaker observed associations between sedentary behaviour and odds for having T2DM. However, the influence of non-response bias on the examined associations is probably small, compared with the selection bias in each individual study, caused by exclusion of a proportion of The Maastricht Study participants, as discussed below. The external validity of The Maastricht Study is limited due age restriction; only adults aged 40-75 years were eligible to participate. In addition, the vast majority of the population was of Caucasian decent. Generalizability to other ages and ethnicities is therefore limited.

In chapter 4, we used data from NHANES, which is designed to provide a representative sample of the US population. By taking into account the complex survey design including sample weights, stratification and clustering, selection bias is reduced through statistical corrections, thus increasing external validity. ${ }^{65}$ Nonetheless, selective non-response may have occurred here as well.

In addition, it is likely that selection bias has also occurred within several studies in this thesis. In all chapters, we performed complete-case analyses, i.e. all participants with missing data on any variable of interest were omitted from the analyses. When missing data occurs completely at random, it is not problematic. ${ }^{66}$ However, for some variables this has not been random. For example, in chapters 3-5, participants with missing CRF 
data were excluded from the analyses, as due to safety concerns, strict exclusion criteria were applied for the exercise test to measure CRF. All participants that reported recent or manifest cardiovascular complications, or that had physical conditions preventing them to perform the test, were deemed ineligible for the test. Consequently, the participants included in these studies did not represent the overall study population, but rather a healthier sub-population. The actual strength of the associations in the total population may in fact be greater than the observed associations. Moreover, some actual associations may not have been observed. For example, in chapter 4 we observed no association between sedentary behaviour and cardio-metabolic health, independent of higher intensity physical activity or CRF. However, this may have been a result of selection bias, as participants with cardio-metabolic complications may have been excluded for the analyses.

\section{Information bias}

Information bias occurs during data collection and is the result of the incorrect determination of exposure and/or outcome variable (measurement error). ${ }^{60,64}$ The potential measurement error of the main variables used in this thesis will be discussed below.

\section{Sedentary behaviour and physical activity}

In all studies in this thesis, daily activities were assessed by accelerometry. In chapter 4 we used the ActiGraph AM-7164 (ActiGraph, Ft. Walton Beach, FL, USA); in the other chapters we used the activPAL3 physical activity monitor (PAL Technologies, Glasgow, UK). As introduced in chapter 1, accelerometers provide data on the intensity and frequency of any movement over a certain time, thus an objective assessment of daily activities is provided. The objective assessment of daily activities overcomes several limitations of self-reported measures such recall bias (a type of information bias). ${ }^{14,15}$ Nonetheless, several issues arise when using these objective data.

The Actigraph has been commonly used to measure the intensity of activities. ${ }^{67}$ Nevertheless, the best cut points to distinguish between different levels of intensity remains a subject of debate. The lack of a consensus on the right cut point could lead to variations in results depending on the chosen cut point. ${ }^{68}$ Further, although the Actigraph is appropriate for measuring intensity of activities it is less accurate in determining a sedentary posture. ${ }^{69,70}$ For instance, standing is often falsely classified as sedentary behaviour. ${ }^{69,71}$ This limitation is surmounted by posture based activity monitors, such as the activPAL.

Because the activPAL utilises information on accelerometer-derived position of the thigh (on which it is placed) it accurately classifies sedentary behaviour, by discriminating a sitting/lying posture from upright postures. ${ }^{72-74}$ As a result, estimates of seden- 
tary time are very precise. However, seated activities with high energy expenditure, such as weight lifting would be incorrectly classified as sedentary behaviour. In addition, in this thesis, acceleration data of the activPAL was not available, which has limited the differentiation of physical activity intensities. We estimated physical activity intensity based on step frequency, which may be a less precise method to determine intensity compared with methods based on acceleration data. However, to determine higher intensity physical activity we applied a step frequency of $>110$ steps/minute. This step frequency has been reported to correspond to a MET score $>3.0,75$ and therefore may be interpreted as an approximation of MVPA. ${ }^{76}$

The reliability of the assessment of sedentary behaviour and physical activity depends also on the number of hours per day and the number of days an accelerometer is worn: wear time.72,77 This is specifically an issue with the ActiGrap, which, in contrast to the activPAL, participants remove before bed time and during water based activies. ${ }^{78}$ Consequently, not all daily activities will be captured and the wear time depends on the participants' adherence to the protocol. To obtain a reliable estimation of daily activities, a minimum wear time of at least 10 hours (as in chapter 4 ) is generally applied.78 However, evidence for this criterion is lacking and studies have shown that a minimum wear time of at least 10 hours provides an underestimation of daily activities compared with a minimum wear time of 12 or 14 hours. ${ }^{79}$ The estimates of sedentary time and MVPA in chapter 4 may therefore be underestimated. The activPAL on the other hand, was worn continuously, 24 hours per day and the final day of wear time was only included when wear time was 14 hours or more. ${ }^{80}$ Therefore, reliable estimates of daily activities from activPAL data were obtained.

To reliably estimate daily activities, in addition to the number of hours per day, the total number of days of accelerometer wear time is important. For correctly estimating sedentary time and MVPA, a minimum of respectively 4 days, and 7 days of wear time appears sufficient. ${ }^{81}$ In addition, 7 days of wear time is regarded as a sufficient number of days to obtain a reproducible measurement of daily actvities. ${ }^{82}$ NHANES and The Maastricht Study both applied a protocol to obtain 7 days of wear time. Compliance to this protocol was high in NHANES and very high in The Maastricht study; respectively, a minimum of $85 \%$ and $95 \%$ of participants, had at least 4 or more days of accelerometer wear time. In all studies in the present thesis we chose to include measurements with 1 day of wear time as well. Our main consideration was to increase the size of our study population. Further, we assumed that if only 1 day of wear time was available, this has occurred mostly at random. In addition, we performed sensitivity analyses in which participants with less than 4 days of wear time were excluded. Overall, this did not change the associations. Therefore, the inclusion of measurements regardless the minimum number of wear days has not affected the observed associations. 


\section{Cardiorespiratory fitness}

Maximum oxygen consumption $\left(\mathrm{VO}_{2 \max }\right)$ obtained with an exercise test up to maximal physical exertion, with direct measurement of oxygen consumption, is currently considered the gold standard for CRF. ${ }^{83}$ However, direct measurement of oxygen consumption is time consuming, costly and technologically challenging. Maximum power output achieved during an exercise test $\left(\mathrm{W}_{\max }\right)$ was therefore used in The Maastricht Study, as an alternative measure of CRF, as it correlates strongly with $\mathrm{VO}_{2 \text { max. }}{ }^{84,85} \mathrm{In}$ addition, exercise tests up to maximal exertion strongly depend on the participants' motivation. An underestimation of $\mathrm{VO}_{2 \max }$ or $\mathrm{W}_{\max }$ will occur when participants are not motivated. Further, due to concerns regarding safety of older or frail participants, maximal exercise testing is often not advisable. ${ }^{86}$ These concerns limit the applicability of maximum exercise tests in large-scale epidemiological studies. As a substitute, submaximal exercise test, such as those used in this thesis, can be used to estimate $\mathrm{VO} 2_{\max }$ or $W_{\max }$.

Many different sub-maximal exercise protocols exist, but the vast majority of these protocols are based on the assumed linear relation between oxygen consumption, power output and heart rate. ${ }^{87,88}$ Generally, participants are instructed to exercise up to a certain heart rate. In The Maastricht Study protocol up to $85 \%$ of age predicted maximum heart rate (220-age) and in the NHANES protocol up to $75 \%$. Using individual linear regression models, sub-maximal values of heart rate and oxygen consumption or power output are extrapolated to a maximum age predicted heart rate and a corresponding value of oxygen consumption $\left(=\mathrm{VO}_{2 \max }\right)$ or power output $\left(=\mathrm{W}_{\max }\right)$. Certain medical conditions, such as diabetic autonomic neuropathy and the use of specific medication (e.g. beta-blockers) are known to affect the linear relationship between heart rate and power output. ${ }^{89}$ For sub-maximal protocols based on heart rate, participants with such conditions are excluded (as in the NHANES protocol), resulting in selection bias, as previously mentioned. To overcome the issues associated with heart rate, some sub-maximal protocols use rating of perceived exertion in place of (or in addition to) heart rate (as in The Maastricht Study). Commonly, the Borg-scale, an interval scale ranging from 6 ('no exertion at all') up to 20 ('maximal exertion') is used to assess rating of perceived exertion. A linear relation between rating of perceived exertion and power output or oxygen consumption may also be assumed. ${ }^{89}$

An important limitation of sub-maximal testing is that it is based on several assumptions: the maximum heart rate is known and there is a linear relation between work load, heart rate and rating of perceived exertion (RPE). However, it is known that estimates of maximum heart rate are subject to error: differences of 8-15 beats per minute were found between estimated (220-age) and measured maximum heart rate. ${ }^{90}$ Further, at low intensity and towards maximum intensity the association between heart rate and 
power output is curve-linear. ${ }^{86,87}$ In addition, rating of perceived exertion is more accurately determined after one or more familiarisation trials. ${ }^{99}$ However, due to time constraints, no such trial was performed in The Maastricht Study. Consequently, participants may have been insufficiently familiar with the Borg-scale, limiting the accuracy of the estimation of $W_{\max }$ based on ratings of perceived exertion. Further, the error of the estimation of $\mathrm{VO}_{2 \max }$ or $\mathrm{W}_{\max }$ will increase, if extrapolations are made from lower sub maximal values (as demonstrated in supplements of chapter 3). For this reason, we excluded tests from the analyses in Maastricht Study if a certain threshold of heart rate or perceived exertion was not achieved. In NHANES however, the exercise protocol differed between participants based on estimated fitness level. After completion of the protocol, the estimated $\mathrm{VO}_{2 \max }$ was not controlled for achieved heart rate, limiting the validity of the estimated $\mathrm{VO}_{2 \max }$.

In conclusion, accuracy of the measurement of CRF from sub-maximal exercise tests is lower than from maximum exercise tests, limiting the accuracy to detect change on intra-individual level. However, it is suitable to distinguish between groups on a population level, due to the quantity of measurements. Since low CRF has been strongly associated with (future) adverse health, sub-maximal exercise tests may provide valuable data in predicting health outcomes and designing preventive strategies.

\section{Confounding and overadjustment}

Arguably the greatest threat for the internal validity of observational studies is confounding. ${ }^{61}$ Confounding occurs when a certain exposure is related to outcome, but a third factor explains or blurs this relation. A confounding variable is related to both the exposure variable as well as the outcome variable, but is not an intermediate variable (does not lie in causal pathway between exposure and outcome). ${ }^{64}$ To overcome bias from confounding, we adjusted for several potential confounding variables using multivariate analyses in all studies. We were able to adjust for many potential confounders as a result of the extensive phenotyping of the characteristics of the study population in all studies. Nevertheless, residual confounding cannot be ruled out. Potential confounders may depend on the outcome studied, yet all analyses relating sedentary behaviour to any outcome were adjusted for higher intensity physical activity, in order to examine if the associations of sedentary behaviour were independent of the amount of higher intensity physical activity. Noteworthy, we did not adjust our analyses for light intensity physical activity. Our motivation for this is multicollinearity in the statistical models: the amount of sedentary time and light intensity physical activity are strongly related. Further, if associations were observed between the amount of sedentary time with an outcome, adjusted for higher intensity physical activity, sedentary time reflects the inverse of standing and light intensity physical activity. Consequently, effects of a 
large amount of sedentary may partly represent the effects of low amounts of standing and light intensity activities.

Adjustment for variables that act as mediators (i.e. factors that lie in causal pathway between exposure and outcome), may lead to the underestimation of observed associations: overadjustment. ${ }^{91}$ An example of (potential) overadjustment in our studies is the adjustment for adiposity (BMI or percentage of body fat). For example, sedentary behaviour has been associated with adiposity (e.g. chapters 5 and 6) and adiposity in turn has been associated with increased risk for T2DM. Consequently, adjusting for adiposity may lead to overadjustment. It is often difficult to determine if a variable acts as a confounder, a mediator, or perhaps both. Especially, when examining outcomes that have a complex aetiology, such as T2DM. Thus, it is not unlikely that some of the associations described in this thesis are subject to overadjustment. However, to minimise the bias caused by confounding we opted to accept the effects of overadjustment, increasing the plausibility of our results. On the other hand, due to potential overadjustment some associations may not have been observed.

\section{Statistical analyses}

Obviously, while awake, reductions in sedentary time will result in increased physical activity, be that of light, moderate or high intensity (less time spent in one activity will always results in more time spent in another activity). Therefore, statistical models in which sedentary time is simply adjusted for other type of activities do not represent real life. Models that are more realistic are isotemporal substitution models (as applied in chapter 3 and 6), in which the effects of reducing one type of activity are analysed as an increase in time spent in another activity. Our results, and results from other studies applying an isotemporal substitution analyses, consistently show beneficial effects of replacing time spent in sedentary behaviour with time spent in physical activity (of any intensity). ${ }^{92-94}$ Even more realistic are complex analyses, such as compositional data analyses in which the distribution between daily activities is used. Results from such analyses also stress the importance of replacing sedentary behaviour with physical activities. $^{95}$

In conclusion from the methodological considerations, we have assessed daily activities with objective measures, which provide a more reliable measure of daily activities than when assessed by self-report. Moreover, in The Maastricht Study, we used the best available activity monitor to measure posture and with a novel approach, we collected data on sedentary behaviour and physical activity 24 hours a day. Further, data on CRF are scarce in large-scale epidemiological studies and may provide important insights for the prediction of disease and mortality. Nevertheless, the measurements used in this thesis are subject to some degree of error and the study design and the possibility 
of residual confounding or overadjustment yield some additional limitations. Therefore, the observations made in this thesis should be confirmed in future studies.

\section{Implications for public health}

In the first part of this thesis (chapters 2 and 3), we observed that physical activity, in particular higher intensity physical activity, was associated with better physical function and CRF. Further, we observed that a large amount of sedentary time was associated with lower CRF and weakly associated with lower physical function, independent of the amount of higher intensity physical activity. The observed associations add to the current evidence linking physical activity, in particular at higher intensity, to improved physical function and CRF. In addition, these associations imply that reducing the amount of sedentary time could lead to improved CRF, although replacing sedentary time with standing was not associated with higher CRF (chapter 3). Further, reducing the amount of sedentary time would only marginally affect physical function (chapter 2). Together these results suggest that a certain threshold of intensity of activity is required to stimulate changes needed to improve physical function and CRF.

In the second part of this thesis, we observed that in subjects aged 40-75 years of The Maastricht Study, a large amount of higher intensity physical activity, a high CRF, and a low amount of sedentary time, were each associated with better cardio-metabolic health, independent of each other (chapters 5 and 6). These observations suggest that in addition to the positive effects associated with MVPA and CRF, reducing the amount of sedentary time could have health benefits. However, we should also consider that in a relatively young and healthy population (NHANES), the amount of sedentary time is of less importance for cardio-metabolic health, than the amount of MVPA and CRF (chapter 4).The effect of age on the associations of sedentary time on cardio-metabolic health should be studied further in a longitudinal setting. Possibly, the effects of prolonged sedentary time on cardio-metabolic health manifest at a later age. Thus, reducing the amount of sedentary time at younger age may still be relevant for reducing the risk for cardio-metabolic diseases.

Overall, the results from our studies underline the importance of current physical activity guidelines, which encourage people to engage in MVPA in order to prevent diseases. In addition, several guidelines have additive recommendations on activities aimed at increasing CRF. For instance in the Netherlands, the 'fit norm' states that in order to improve and maintain CRF, everyone should engage three times per week, 20 minutes in vigorous activities. ${ }^{28}$ Our results underscore the importance of higher intensity physical activity as determinant of CRF. Further, since CRF showed strong associations with the risk of having the metabolic syndrome and T2DM, the recommendation to improve 
CRF seems to be an important addition in the guidelines. In chapter 5 , a shift from low to medium CRF was associated with greatest reduction in risk of having the metabolic syndrome and T2DM. This great increase implies that in particular people with low CRF should be encouraged to increase their level of CRF by engaging more in higher intensity activities. Further, even in people with high CRF, a large amount of sedentary time was associated with a higher risk for having the metabolic syndrome and T2DM (chapter 5). Thus, regardless of level of CRF, people may benefit from reducing the amount of sedentary time.

However, as mentioned, our studies are limited by their cross-sectional design and true causality and effect sizes cannot be determined. Nonetheless, even if true effect sizes of reducing sedentary behaviour would be small, on a population level it may still be relevant, since the majority of the population spends most time of the day in sedentary behaviour.9-11 Health effects of reducing sedentary time could be especially meaningful for people that have difficulties (or are unable) to engage in MVPA, such as those with chronic illnesses or functional impairments.

Messages to 'sit less' or 'move more' throughout the day imply the same: decrease the amount of sedentary time and increase the amount of light intensity physical activity. From a public health perspective, it does not actually matter what the underlying mechanism is (sedentary behaviour indicates positive energy balance due to lack of light intensity physical activities (or NEAT), or sedentary behaviour acts via distinct signalling pathways, or a combination of both). Note that improved insight into mechanisms of prolonged sitting is needed for concrete advice; for example, avoid prolonged sedentary bouts in excess of one hour. An increasing number of studies (including the studies in this thesis) have demonstrated a deleterious effect of large amounts of sedentary time on cardio-metabolic health, supported by findings in experimental settings. ${ }^{46-50}$ Altogether, these findings suggest health benefits of reducing sedentary behaviour. Therefore, recommendations on reducing sedentary behaviour should be developed. Of note, these should be an addition to guidelines on the recommendations of MVPA (and CRF). Possibly, due to the relatively low effort required, public health messages to sit less can be more successful than messages to engage more in MVPA, which requires more effort. In addition, reducing sedentary behaviour can be facilitated in many settings (transport, public places, work places, schools etc.) supporting people to sit less. Further, recent technological developments of consumer-based wearable activity monitors (such as the Fitbit) and applications on smartphones, may provide individuals with insight into their daily activities and aid in improving their activity pattern. 
Some countries have already adopted recommendations on sedentary behaviour in their physical activity guidelines.96,97 In addition, since the reported health effects of reducing sedentary behaviour include improved glucose metabolism, organisations such as the Dutch Diabetes Foundation, have started encouraging patients with diabetes to sit less. ${ }^{98}$ However, at this stage it is unclear what the specific recommendations on sedentary behaviour should state, as insights are lacking into how much sedentary time is too much.

\section{Future research}

The relatively recent implementation of accelerometers to measure physical activity and sedentary behaviour in large population based studies has provided researchers with more reliable and valid estimates of daily physical activities. In most of these studies (including the studies in this thesis) associations between sedentary behaviour and physical activity with health outcomes have been examined cross-sectionally. ${ }^{99}$ In the future, research is needed to establish the longitudinal associations of (patterns of) sedentary behaviour and physical activity with health outcomes in order to determine causality. Specifically, these longitudinal studies should focus on the dose-response relationship between sedentary behaviour and physical activity with health. In other words, how much physical activity do we need and at what intensity, or how much sedentary time should we avoid in order to prevent diseases? Ideally, this should also be studied in experimental or randomized control trials where the frequency, amount, intensity, and timing of sedentary behaviour and physical activity are regulated. These types of trails also lead to an increased understanding of the health effects of the pattern in which sedentary behaviour and other activities are accumulated. Insight into dose-response relation and daily activity pattern is needed for enabling intervention studies to set aims regarding the amount of sedentary time to reduce and physical activity to increase. In addition, this information is crucial for the development of public health messages and to update current physical activity guidelines, which are primarily based on self-reported data. Most guidelines lack recommendations on sedentary behaviour and light intensity physical activity; data that is not reliable when selfreported. In ongoing and future studies, researchers have the potential to measure and comprehensively analyse all daily activities using accelerometers, improving the understanding of health effects of physical activity and sedentary behaviour. ${ }^{100}$

The objective measurement of physical activity also poses challenges. Accelerometers capture a vast amount of data. Researchers have begun to develop techniques to process all these data and to reduce these data to several exposure variables (such as mean amount of sedentary time per day, and mean number of sedentary breaks per day). A challenge is the variability in types of accelerometers. The site of placement (e.g. wrist, 
hip, and thigh) differs between monitors. In addition, to differentiate intensity of physical activity into sedentary behaviour, and light to vigorous physical activity, many studies have used cut points for acceleration data. However, these cut points differ between studies and devices used, limiting comparability. Furthermore, these cut points may differ by the population being studied. Calibration studies in different populations, with different devices are required to optimize generalizability and interstudy comparability. Optimising generalizability is desirable if comparisons in physical activity level between populations or countries are made. Another issue is that sedentary behaviour pattern variables are currently not standardised. For example, there is no standardised definition of a sedentary break. Should a sedentary break have a minimum duration or a minimum (or perhaps maximum) intensity? Without this additional information, it is unclear what it represents or should represent: the frequency of sedentary bouts, a measure of non-sedentary time? Fundamental research is needed to standardize constructs to express the pattern of sedentary behaviour. Moreover, reducing the vast amount of data into a few 'simple' exposure measures may be undesirable, as much information on patterns of physical activity is neglected. As an alternative technique, pattern recognition, that utilises more of the raw acceleration data, may show promise in the near future. ${ }^{101}$ Clearly, much work is needed in order to utilise the full potential of objective physical activity data collected in different studies.

Studies should also examine if there is a certain threshold of physical activity that attenuates or even eliminates the adverse health effects of large amounts of sedentary time. A recent meta-analysis has shown that a very high level of MVPA (in excess of 1 hour per day) eliminates the deleterious effects of sedentary time. However, sedentary time and physical activity were based on self-report. ${ }^{102}$ In addition, other health related factors such as CRF should be considered as well. It may be possible that a (very) high CRF eliminates the adverse health effects of large amounts of sedentary time. Also, as behaviours do not occur independent of each other, studies should examine combined associations (as we did in chapter 5). Analyses of combined associations provide insight of potential clustering of risk factors around certain combinations of behaviours, such as low CRF in combination with high sedentary time.

In order to successfully reduce the amount of time spent in sedentary behaviour, and to increase time spent in MVPA, future studies should examine how this can be achieved. As activity monitors lack information on the location or setting of individual activities, combining the data from activity monitors (on frequency and intensity) with selfreported data (on where and when) may provide insight into the setting that most sedentary behaviour or MVPA occurs. ${ }^{14,72,103}$ Improved understanding of these settings may allow to tailor interventions to certain subgroups, improving the chance of successfully influencing daily activities. Currently, many interventions aimed at improv- 
ing MVPA are successful on the short-term. However, the long-term effects are often attenuated. ${ }^{104,105}$ When interventions are better tailored to an individuals' daily routine, this may improve the long-term effectiveness. For example, during work time, the amount of activity will largely depend on the type of work a person does, while during leisure time a person is (at least to a certain extent) free to choose his/her activities. Interventions in work place setting provide some evidence that during working hours, a reduction in sedentary time or an increase in physical activity is attainable. However, at this stage there is insufficient evidence that these workplace interventions result in improved cardio-metabolic health. ${ }^{106}$ On the other hand, sedentary time and physical activity during leisure time may be more relevant for health risk, as the chosen activity pattern may cluster with other health related behaviours, such as higher caloric intake. ${ }^{107}$ Thus, subjective measures of daily activities may provide some information that (currently) is unavailable from objective measures.

Lastly, as previously mentioned, studies are needed to establish the mechanisms and biological pathways that explain how sedentary behaviour affects health. In particular, these studies should examine if these pathways are indeed distinct from those of MVPA. Improved insight into these mechanisms may also be derived from observational research examining associations of sedentary behaviour with variables that are known to be important in the aetiology of cardio-metabolic diseases, for example microvascular dysfunction, endothelial dysfunction, advanced glycation end products (AGEs), and more detailed variables of body composition such as visceral adipose tissue.

\section{Conclusion}

The studies in this thesis emphasize that physical activity, in particular of higher intensity, is an important factor in improving or maintaining physical function, CRF, and cardio-metabolic health. In addition, CRF itself was strongly associated with cardiometabolic health. Further, our studies show that reducing the amount of daily sedentary time may have an additional effect on cardio-metabolic health, regardless of the amount of higher intensity physical activity and CRF, in particular in middle aged and elderly individuals (40-75 years). This suggests that in order to prevent cardiometabolic diseases, next to engaging regularly in moderate and higher intensity physical activity (coinciding with higher CRF), a certain balance between sedentary behaviour, and light intensity physical activity is required. Future studies are needed to provide information on how much sedentary time to avoid and how much physical activity we need and at what intensity and frequency, in order to prevent diseases. 


\section{References}

1. Perk J, De Backer G, Gohlke H, Graham I, Reiner Ž, Verschuren M, Albus C, Benlian P, Boysen G, Cifkova R. European Guidelines on cardiovascular disease prevention in clinical practice (version 2012). Eur. Heart J. 2012;33(13):1635-1701.

2. World Health Organization \& UNAIDS. Prevention of cardiovascular disease. 2007.

3. Lee I-M, Shiroma EJ, Lobelo F, Puska P, Blair SN, Katzmarzyk PT, Lancet-Physical-Activity-SeriesWorking-Group. Effect of physical inactivity on major non-communicable diseases worldwide: an analysis of burden of disease and life expectancy. The lancet 2012;380(9838):219-229.

4. Warburton DE, Bredin SS. Reflections on physical activity and health: what should we recommend? Can. J. Cardiol. 2016;32(4):495-504.

5. Kahlmeier S, Wijnhoven TMA, Alpiger P, Schweizer C, Breda J, Martin BW. National physical activity recommendations: systematic overview and analysis of the situation in European countries. BMC Public Health 2015;15(1):1-14.

6. World Health Organization. Global recommendations on physical activity for health. 2010.

7. Young DR, Hivert M-F, Alhassan S, Camhi SM, Ferguson JF, Katzmarzyk PT, Lewis CE, Owen N, Perry CK, Siddique J. Sedentary Behavior and Cardiovascular Morbidity and Mortality: A Science Advisory From the American Heart Association. Circulation 2016;134(13):e262-e279.

8. Sedentary-Behaviour-Research-Network. (2102). Letter to the editor: standardized use of the terms "sedentary" and "sedentary behaviours". Appl. Physiol. Nutr. Metab;37:540-542.

9. Matthews CE, Chen KY, Freedson PS, Buchowski MS, Beech BM, Pate RR, Troiano RP. Amount of time spent in sedentary behaviors in the United States, 2003-2004. Am. J. Epidemiol. 2008;167(7):875881.

10. Dunstan DW, Howard B, Healy GN, Owen N. Too much sitting-a health hazard. Diabetes Res. Clin. Pract. 2012;97(3):368-376.

11. Bennie JA, Chau JY, van der Ploeg HP, Stamatakis E, Do A, Bauman A. The prevalence and correlates of sitting in European adults-a comparison of 32 Eurobarometer-participating countries. Int. J. Behav. Nutr. Phys. Act. 2013;10(1):1.

12. Owen N, Healy GN, Matthews CE, Dunstan DW. Too much sitting: the population health science of sedentary behavior. Exerc. Sport Sci. Rev. 2010;38(3):105-13.

13. Hamilton MT, Healy GN, Dunstan DW, Zderic TW, Owen N. Too little exercise and too much sitting: inactivity physiology and the need for new recommendations on sedentary behavior. Curr Cardiovasc Risk Rep 2008;2(4):292-298.

14. Atkin AJ, Gorely T, Clemes SA, Yates T, Edwardson C, Brage S, Salmon J, Marshall SJ, Biddle SJ. Methods of measurement in epidemiology: sedentary behaviour. Int. J. Epidemiol. 2012;41(5):14601471.

15. Healy GN, Clark BK, Winkler EA, Gardiner PA, Brown WJ, Matthews CE. Measurement of adults' sedentary time in population-based studies. Am. J. Prev. Med. 2011;41(2):216-227.

16. Bauman A, Merom D, Bull FC, Buchner DM, Singh MAF. Updating the evidence for physical activity: summative reviews of the epidemiological evidence, prevalence, and interventions to promote "Active Aging". The Gerontologist 2016;56(Suppl 2):S268-S280.

17. Lin X, Zhang X, Guo J, Roberts CK, McKenzie S, Wu WC, Liu S, Song Y. Effects of Exercise Training on Cardiorespiratory Fitness and Biomarkers of Cardiometabolic Health: A Systematic Review and Meta-Analysis of Randomized Controlled Trials. J Am Heart Assoc 2015;4(7):e002014.

18. Warburton DE, Charlesworth S, Ivey A, Nettlefold L, Bredin SS. A systematic review of the evidence for Canada's Physical Activity Guidelines for Adults. Int. J. Behav. Nutr. Phys. Act. 2010;7(1):1.

19. Garatachea N, Pareja-Galeano H, Sanchis-Gomar F, Santos-Lozano A, Fiuza-Luces C, Morán M, Emanuele E, Joyner MJ, Lucia A. Exercise attenuates the major hallmarks of aging. Rejuvenation Res. 2015;18(1):57-89.

20. Lavie CJ, Arena R, Swift DL, Johannsen NM, Sui X, Lee D-c, Earnest CP, Church TS, O'Keefe JH, Milani RV. Exercise and the cardiovascular system. Circ. Res. 2015;117(2):207-219.

21. McArdle WD, Katch FI, Katch VL. Exercise physiology: nutrition, energy, and human performance Lippincott Williams \& Wilkins, 2010. 
22. Irrcher I, Adhihetty PJ, Joseph A-M, Ljubicic V, Hood DA. Regulation of mitochondrial biogenesis in muscle by endurance exercise. Sports Med. 2003;33(11):783-793.

23. Bouchard C, Blair SN, Katzmarzyk PT. Less sitting, more physical activity, or higher fitness? Mayo Clin. Proc.: Elsevier, 2015;1533-1540.

24. Thompson PD, Crouse SF, Goodpaster B, Kelley D, Moyna N, Pescatello L. The acute versus the chronic response to exercise. Med. Sci. Sports Exerc. 2001;33(6 Suppl):S438-45; discussion S452-3.

25. Lee D-C, Pate RR, Lavie CJ, Sui X, Church TS, Blair SN. Leisure-time running reduces all-cause and cardiovascular mortality risk. J. Am. Coll. Cardiol. 2014;64(5):472-481.

26. Jefferis BJ, Parsons TJ, Sartini C, Ash S, Lennon LT, Wannamethee SG, Lee I-M, Whincup PH. Does duration of physical activity bouts matter for adiposity and metabolic syndrome? A cross-sectional study of older British men. Int. J. Behav. Nutr. Phys. Act. 2016;13(1):36.

27. Haskell WL, Lee I-M, Pate RR, Powell KE, Blair SN, Franklin BA, Macera CA, Heath GW, Thompson PD, Bauman A. Physical activity and public health: updated recommendation for adults from the American College of Sports Medicine and the American Heart Association. Circulation 2007;116(9):1081.

28. Hildebrandt. VH, Ooijendijk WTM, Hopman-Rock M. Trendrapport Bewegen en Gezondheid 2006/2007. . 2008.

29. Tjønna AE, Lee SJ, Rognmo Ø, Stølen TO, Bye A, Haram PM, Loennechen JP, Al-Share QY, Skogvoll E, Slørdahl SA. Aerobic interval training versus continuous moderate exercise as a treatment for the metabolic syndrome. Circulation 2008;118(4):346-354.

30. Helgerud J, Hoydal K, Wang E, Karlsen T, Berg P, Bjerkaas M, Simonsen T, Helgesen C, Hjorth N, Bach R. Aerobic High-Intensity Intervals Improve VO 2 $\mathrm{m} \sim \mathrm{a} \sim \mathrm{x}$ More Than Moderate Training. Med. Sci. Sports Exerc. 2007;39(4):665.

31. Brocklebank LA, Falconer CL, Page AS, Perry R, Cooper AR. Accelerometer-measured sedentary time and cardiometabolic biomarkers: A systematic review. Prev. Med. 2015;76:92-102.

32. Rezende LFM, Rey-López JP, Matsudo VKR, Luiz OC. Sedentary behavior and health outcomes among older adults: a systematic review. BMC Public Health 2014;14(1):333.

33. Edwardson CL, Gorely T, Davies MJ, Gray LJ, Khunti K, Wilmot EG, Yates T, Biddle SJ. Association of sedentary behaviour with metabolic syndrome: a meta-analysis. PLoS ONE 2012;7(4):e34916.

34. Ford ES, Caspersen CJ. Sedentary behaviour and cardiovascular disease: a review of prospective studies. Int. J. Epidemiol. 2012;41(5):1338-53.

35. Wilmot EG, Edwardson CL, Achana FA, Davies MJ, Gorely T, Gray LJ, Khunti K, Yates T, Biddle SJ. Sedentary time in adults and the association with diabetes, cardiovascular disease and death: systematic review and meta-analysis. Diabetologia 2012;55(11):2895-2905.

36. Chau JY, Grunseit AC, Chey T, Stamatakis E, Brown WJ, Matthews CE, Bauman AE, van der Ploeg HP. Daily sitting time and all-cause mortality: a meta-analysis. PLoS ONE 2013;8(11):e80000.

37. Levine JA, Vander Weg MW, Hill JO, Klesges RC. Non-exercise activity thermogenesis. Arterioscler. Thromb. Vasc. Biol. 2006;26(4):729-736.

38. Levine JA. Non-exercise activity thermogenesis (NEAT). Nutr. Rev. 2004;62(suppl 2):S82-S97.

39. Maher C, Olds T, Mire E, Katzmarzyk PT. Reconsidering the sedentary behaviour paradigm. PLoS ONE 2014;9(1):e86403.

40. Balkau B, Mhamdi L, Oppert J-M, Nolan J, Golay A, Porcellati F, Laakso M, Ferrannini E. Physical activity and insulin sensitivity. Diabetes 2008;57(10):2613-2618.

41. Booth FW, Chakravarthy MV, Spangenburg EE. Exercise and gene expression: physiological regulation of the human genome through physical activity. The Journal of physiology 2002;543(2):399411.

42. Thijssen DH, Green DJ, Hopman MT. Blood vessel remodeling and physical inactivity in humans. J. Appl. Physiol. 2011;111(6):1836-1845.

43. Thosar SS, Johnson BD, Johnston JD, Wallace JP. Sitting and endothelial dysfunction: the role of shear stress. Medical Science Monitor 2012;18(12):REV173-REV180.

44. Hamilton MT, Hamilton DG, Zderic TW. Role of low energy expenditure and sitting in obesity, metabolic syndrome, type 2 diabetes, and cardiovascular disease. Diabetes 2007;56(11):2655-2667.

45. Hawley J, Gibala M. What's new since Hippocrates? Preventing type 2 diabetes by physical exercise and diet. Diabetologia 2012;55(3):535-539. 
46. Dunstan DW, Kingwell BA, Larsen R, Healy GN, Cerin E, Hamilton MT, Shaw JE, Bertovic DA, Zimmet PZ, Salmon J. Breaking up prolonged sitting reduces postprandial glucose and insulin responses. Diabetes Care 2012;35(5):976-983.

47. Peddie MC, Bone JL, Rehrer NJ, Skeaff CM, Gray AR, Perry TL. Breaking prolonged sitting reduces postprandial glycemia in healthy, normal-weight adults: a randomized crossover trial. Am. J. Clin. Nutr. 2013;98(2):358-366.

48. Henson J, Davies MJ, Bodicoat DH, Edwardson CL, Gill JM, Stensel DJ, Tolfrey K, Dunstan DW, Khunti K, Yates T. Breaking up prolonged sitting with standing or walking attenuates the postprandial metabolic response in postmenopausal women: a randomized acute study. Diabetes Care 2016;39(1):130-138.

49. Duvivier BM, Schaper NC, Bremers MA, van Crombrugge G, Menheere PP, Kars M, Savelberg HH. Minimal Intensity Physical Activity (Standing and Walking) of Longer Duration Improves Insulin Action and Plasma Lipids More than Shorter Periods of Moderate to Vigorous Exercise (Cycling) in Sedentary Subjects When Energy Expenditure Is Comparable. PLoS ONE 2013;8(2):e55542.

50. Duvivier BM, Schaper NC, Hesselink MK, van Kan L, Stienen N, Winkens B, Koster A, Savelberg $\mathrm{HH}$. Breaking sitting with light activities vs structured exercise: a randomised crossover study demonstrating benefits for glycaemic control and insulin sensitivity in type 2 diabetes. Diabetologia 2016:1-9.

51. Lee D, Sui X, Ortega F, Kim Y, Church T, Winett R, Ekelund U, Katzmarzyk P, Blair S. Comparisons of leisure-time physical activity and cardiorespiratory fitness as predictors of allcause mortality in men and women. Br. J. Sports Med. 2011;45(6):504-10.

52. Sassen B, Cornelissen VA, Kiers H, Wittink H, Kok G, Vanhees L. Physical fitness matters more than physical activity in controlling cardiovascular disease risk factors. Eur. J. Cardiovasc. Prev. Rehabil. 2009;16(6):677-683.

53. Minder CM, Shaya GE, Michos ED, Keenan TE, Blumenthal RS, Nasir K, Carvalho JA, Conceição RD, Santos RD, Blaha MJ. Relation between self-reported physical activity level, fitness, and cardiometabolic risk. Am. J. Cardiol. 2014;113(4):637-643.

54. Ekblom Ö, Ekblom-Bak E, Rosengren A, Hallsten M, Bergström G, Börjesson M. Cardiorespiratory fitness, sedentary behaviour and physical activity are independently associated with the metabolic syndrome, results from the SCAPIS pilot study. PLoS ONE 2015;10(6):e0131586.

55. Blair SN, Connelly JC. How much physical activity should we do? The case for moderate amounts and intensities of physical activity. Res. Q. Exerc. Sport 1996;67(2):193-205.

56. Bouchard C, Rankinen T. Individual differences in response to regular physical activity. Med. Sci. Sports Exerc. 2001;33(6 Suppl):S446-51; discussion S452-3.

57. Gill JM, Celis-Morales CA, Ghouri N. Physical activity, ethnicity and cardio-metabolic health: does one size fit all? Atherosclerosis 2014;232(2):319-333.

58. Lee DC, Artero EG, Sui X, Blair SN. Review: Mortality trends in the general population: the importance of cardiorespiratory fitness. J. Psychopharmacol. 2010;24(4 suppl):27-35.

59. Porta M. A dictionary of epidemiology Oxford university press, 2014.

60. Tripepi G, Jager KJ, Dekker FW, Zoccali C. Selection bias and information bias in clinical research. Nephron Clin. Pract. 2010;115(2):c94-c99.

61. Rothman KJ, Greenland S, Lash TL. Modern epidemiology Lippincott Williams \& Wilkins, 2008.

62. Hernán MA, Hernández-Díaz S, Robins JM. A structural approach to selection bias. Epidemiology 2004;15(5):615-625.

63. Schram MT, Sep SJ, van der Kallen CJ, Dagnelie PC, Koster A, Schaper N, Henry RM, Stehouwer $\mathrm{CD}$. The Maastricht Study: an extensive phenotyping study on determinants of type 2 diabetes, its complications and its comorbidities. Eur. J. Epidemiol. 2014;29(6):439-51.

64. Grimes DA, Schulz KF. Bias and causal associations in observational research. The Lancet 2002;359(9302):248-252.

65. National Center for Health Statistics. Introduction to NHANES. [cited February 2017] Available from: https://www.cdc.gov/Nchs/Nhanes/about_nhanes.htm

66. Donders ART, van der Heijden GJ, Stijnen T, Moons KG. Review: a gentle introduction to imputation of missing values. J. Clin. Epidemiol. 2006;59(10):1087-1091.

67. Troiano RP, Berrigan D, Dodd KW, Masse LC, Tilert T, McDowell M. Physical activity in the United States measured by accelerometer. Med. Sci. Sports Exerc. 2008;40(1):181. 
68. Gorman E, Hanson H, Yang P, Khan K, Liu-Ambrose T, Ashe M. Accelerometry analysis of physical activity and sedentary behavior in older adults: a systematic review and data analysis. Eur Rev Aging Phys Act 2013;11(1):35.

69. Bassett DR. Device-based monitoring in physical activity and public health research. Physiol. Meas. 2012;33(11):1769.

70. Aguilar-Farías N, Brown WJ, Peeters GG. ActiGraph GT3X+ cut-points for identifying sedentary behaviour in older adults in free-living environments. J. Sci. Med. Sport 2014;17(3):293-299.

71. Koster A, Shiroma EJ, Caserotti P, Matthews CE, Chen KY, Glynn NW, Harris TB. Comparison of Sedentary Estimates between activPAL and Hip-and Wrist-Worn ActiGraph. Med. Sci. Sports Exerc. 2016;48(8):1514-1522.

72. Kang M, Rowe DA. Issues and challenges in sedentary behavior measurement. Meas Phys Educ Exerc Sci 2015;19(3):105-115.

73. Kozey-Keadle S, Libertine A, Lyden K, Staudenmayer J, Freedson PS. Validation of wearable monitors for assessing sedentary behavior. Med. Sci. Sports Exerc. 2011;43(8):1561-7.

74. Godfrey A, Culhane K, Lyons G. Comparison of the performance of the activPAL ${ }^{\mathrm{TM}}$ Professional physical activity logger to a discrete accelerometer-based activity monitor. Med. Eng. Phys. 2007;29(8):930-934.

75. Tudor-Locke C, Rowe DA. Using cadence to study free-living ambulatory behaviour. Sports Med. 2012;42(5):381-398.

76. Ainsworth BE, Haskell WL, Whitt MC, Irwin ML, Swartz AM, Strath SJ, O Brien WL, Bassett DR, Schmitz KH, Emplaincourt PO. Compendium of physical activities: an update of activity codes and MET intensities. Med. Sci. Sports Exerc. 2000;32(9; SUPP/1):S498-S504.

77. Edwardson CL, Winkler EA, Bodicoat DH, Yates T, Davies MJ, Dunstan DW, Healy GN. Considerations when using the activPAL monitor in field-based research with adult populations. Journal of Sport and Health Science 2016.

78. Tudor-Locke C, Camhi SM, Troiano RP. A catalog of rules, variables, and definitions applied to accelerometer data in the National Health and Nutrition Examination Survey, 2003-2006. Prev. Chronic. Dis. 2012;9:110332.

79. Herrmann SD, Barreira TV, Kang M, Ainsworth BE. Impact of accelerometer wear time on physical activity data: a NHANES semisimulation data approach. Br. J. Sports Med. 2014;48(3):278-282.

80. van der Berg JD, Willems PJ, van der Velde JHPM, Savelberg HHCM, Schaper NC, Schram MT, Sep SJ, Dagnelie PC, Bosma H, Stehouwer CDA. Identifying waking time in 24-h accelerometry data in adults using an automated algorithm. J. Sports Sci. 2016;34(19):1867-73.

81. Barreira TV, Hamilton MT, Craft LL, Gapstur SM, Siddique J, Zderic TW. Intra-individual and inter-individual variability in daily sitting time and MVPA. J. Sci. Med. Sport 2016;19(6):476-481.

82. Kozey-Keadle S, Shiroma EJ, Kamada M, Matthews CE, Harris TB, Lee I-M. Reproducibility of Accelerometer-Assessed Physical Activity and Sedentary Time. Am. J. Prev. Med. 2017.

83. American College of Sports Medicine. ACSM's guidelines for exercise testing and prescription Lippincott Williams \& Wilkins, 2013.

84. Storer TW, Davis JA, Caiozzo VJ. Accurate prediction of VO2max in cycle ergometry. Med. Sci. Sports Exerc. 1990;22(5):704-12.

85. Patton J, Vogel J, Mello R. Evaluation of a maximal predictive cycle ergometer test of aerobic power. Eur. J. Appl. Physiol. 1982;49(1):131-140.

86. Noonan V, Dean E. Submaximal exercise testing: clinical application and interpretation. Phys. Ther. 2000;80(8):782-807.

87. Sartor F, Vernillo G, de Morree HM, Bonomi AG, La Torre A, Kubis H-P, Veicsteinas A. Estimation of maximal oxygen uptake via submaximal exercise testing in sports, clinical, and home settings. Sports Med. 2013;43(9):865-873.

88. Akalan C, Robergs RA, Kravitz L. Prediction of VO2max from an individualized submaximal cycle ergometer protocol. J Exerc Physiol Online 2008;11(2):1-17.

89. Coquart JB, Garcin M, Parfitt G, Tourny-Chollet C, Eston RG. Prediction of maximal or peak oxygen uptake from ratings of perceived exertion. Sports Med. 2014;44(5):563-578.

90. Achten J, Jeukendrup AE. Heart rate monitoring. Sports Med. 2003;33(7):517-538.

91. Schisterman EF, Cole SR, Platt RW. Overadjustment bias and unnecessary adjustment in epidemiologic studies. Epidemiology (Cambridge, Mass.) 2009;20(4):488. 
92. Healy GN, Winkler EA, Owen N, Anuradha S, Dunstan DW. Replacing sitting time with standing or stepping: associations with cardio-metabolic risk biomarkers. Eur. Heart J. 2015:ehv308.

93. Hamer M, Stamatakis E, Steptoe A. Effects of substituting sedentary time with physical activity on metabolic risk. Med. Sci. Sports Exerc. 2014;46(10):1946-1950.

94. Ekblom-Bak E, Ekblom Ö, Bergström G, Börjesson M. Isotemporal substitution of sedentary time by physical activity of different intensities and bout lengths, and its associations with metabolic risk. Eur J Prev Cardiol 2016;23(9):967-974.

95. Chastin SF, Palarea-Albaladejo J, Dontje ML, Skelton DA. Combined effects of time spent in physical activity, sedentary behaviors and sleep on obesity and cardio-metabolic health markers: a novel compositional data analysis approach. PLoS ONE 2015;10(10):e0139984.

96. Australian Government Department of Health. Australia's Physical Activity and Sedentary Behaviour Guidelines For Adults (18-64 years). 2014 [cited February 2017]. Available from: http://www.health.gov.au/internet/main/publishing.nsf/Content/health-pubhlth-strateg-physact-guidelines.

97. Canadian Society of Exercise Physiology. Canadian Physical Activity and Sedentary Behaviour Guidelines. 2012 [cited Febraury 2017]. Available from: www.csep.ca/guidelines.

98. Diabetes Fonds. Gezonder leven, minder zitten. 2016 [cited February 2017] Available from: https://www.diabetesfonds.nl/gezonder-leven/minder-zitten.

99. Lee I-M, Shiroma EJ. Using accelerometers to measure physical activity in large-scale epidemiological studies: issues and challenges. Br. J. Sports Med. 2014;48(3):197-201.

100. Plasqui G, Bonomi A, Westerterp K. Daily physical activity assessment with accelerometers: new insights and validation studies. Obes. Rev. 2013;14(6):451-462.

101. Freedson P, Bowles HR, Troiano R, Haskell W. Assessment of physical activity using wearable monitors: recommendations for monitor calibration and use in the field. Med. Sci. Sports Exerc. 2012;44(1 Suppl 1):S1.

102. Ekelund U, Steene-Johannessen J, Brown WJ, Fagerland MW, Owen N, Powell KE, Bauman A, Lee I-M, Series LPA, Group LSBW. Does physical activity attenuate, or even eliminate, the detrimental association of sitting time with mortality? A harmonised meta-analysis of data from more than 1 million men and women. The Lancet 2016;388(10051):1302-1310.

103. Matthews CE, Hagströmer M, Pober DM, Bowles HR. Best practices for using physical activity monitors in population-based research. Med. Sci. Sports Exerc. 2012;44(1 Suppl 1):S68.

104. Van Der Bij AK, Laurant MG, Wensing M. Effectiveness of physical activity interventions for older adults: a review. Am. J. Prev. Med. 2002;22(2):120-133.

105. Müller-Riemenschneider F, Reinhold T, Nocon M, Willich SN. Long-term effectiveness of interventions promoting physical activity: a systematic review. Prev. Med. 2008;47(4):354-368.

106. Commissaris DA, Huysmans MA, Mathiassen SE, Srinivasan D, Koppes LL, Hendriksen IJ. Interventions to reduce sedentary behavior and increase physical activity during productive work: a systematic review. Scand. J. Work. Environ. Health 2016;42(3):181-191.

107. Cleland VJ, Schmidt MD, Dwyer T, Venn AJ. Television viewing and abdominal obesity in young adults: is the association mediated by food and beverage consumption during viewing time or reduced leisure-time physical activity? Am. J. Clin. Nutr. 2008;87(5):1148-1155. 


\section{Appendices}

Samenvatting (NL)

Valorisation addendum

Dankwoord (NL)

Curriculum Vitae 



\section{Samenvatting}

Cardio-metabole ziektes, zoals hart- en vaatziekten en type 2 diabetes, komen wereldwijd steeds vaker voor. Deze ziektes dragen sterk bij aan verhoogde lasten voor patiënten, maar ook aan kosten voor gezondheidszorg en maatschappij. Uit tientallen jaren onderzoek is gebleken dat voldoende lichamelijke activiteit het risico op cardiometabole ziektes verlaagt. Dit komt (onder andere) doordat bewegen diverse cardiometabole risicofactoren zoals bloeddruk, suikerwaardes, cholesterolwaardes en vetzuurwaardes in het bloed op pijl houdt. Onderzoek naar de positieve effecten van lichamelijke activiteit is voornamelijk gericht op activiteiten van matige tot zware (hogere) intensiteit; waarbij de hartslag en ademhaling zijn verhoogd. Naast een betere cardio-metabole gezondheid, leidt lichamelijke activiteit van hogere intensiteit ook tot beter fysiek functioneren (de mate waarin een persoon om kan gaan met de fysieke eisen van het dagelijks leven) en een betere cardiorespiratoire fitheid (kortweg fitheid, de mate waarin het luchtwegsysteem en het hart- en vaatsysteem tijdens inspanning zuurstof naar de spieren kunnen transporteren). Fysiek functioneren en in het bijzonder fitheid zijn op zichzelf ook sterke voorspellers van cardio-metabole ziektes.

Onderzoek naar de positieve effecten van lichamelijke activiteit is vooral gericht op lichamelijk activiteit van hogere intensiteit. Echter, deze activiteiten omvatten slechts een klein deel van alle dagelijkse lichamelijke activiteit. Het merendeel van de dag bestaat uit sedentair gedrag. Sedentair gedrag wordt gedefinieerd als lichamelijke activiteit met een laag energie verbruik (niet veel hoger dan energieverbruik in rust) in een zittende of liggende houding. Voorbeelden zijn televisie kijken, autorijden, en werken op een PC. Verschillende studies hebben aangetoond dat sedentair gedrag een risicofactor is voor een slechtere cardio-metabole gezondheid, onafhankelijk van de hoeveelheid lichamelijke activiteit van hogere intensiteit. Met andere woorden, ook mensen die regelmatig sporten hebben een verhoogd risico op hart- en vaatziekten en type 2 diabetes als zij het merendeel van de dag zitten. Het is nog onduidelijk of veel zitten ook gepaard gaat met een lagere fitheid en met slechter fysiek functioneren, onafhankelijk van lichamelijk activiteit van hogere intensiteit. Ook is het onduidelijk of de relatie tussen veel zitten en het risico op hart- en vaatziekten en type 2 diabetes blijft bestaan als rekening wordt gehouden met fitheid.

Het eerste doel in deze thesis was om te onderzoeken of sedentair gedrag en lichamelijke activiteit geassocieerd zijn met fysiek functioneren en fitheid. Vervolgens onderzochten we de onafhankelijke en gecombineerde associaties van sedentair gedrag, lichamelijke activiteit en fitheid met cardio-metabole gezondheid. Hiervoor gebruikten we gegevens van twee grote observationele studies: De Maastricht Studie en The National Health and Nutrition Examination Survey (NHANES). In beide studies is ge- 
bruik gemaakt van beweegmeters om de dagelijkse activiteiten van deelnemers te bepalen. Beweegmeters zijn kleine apparaatjes die mensen op het lichaam dragen en die de hoeveelheid, intensiteit en duur van alle bewegingen te registreren. Met deze apparaatjes wordt op een objectieve, en daardoor betrouwbare, manier een overzicht gecreeerd van de dagelijkse hoeveelheid sedentair gedrag en lichamelijke activiteit. Dit is een duidelijke verbetering ten opzichte van eerdere grote observationele studies waarin lichamelijke activiteit en sedentair gedrag veelal met vragenlijsten werd gemeten.

In onderstaande paragrafen wordt per hoofdstuk een korte samenvatting gegeven van de bevindingen en daaropvolgend een algemene conclusie.

\section{Hoofdstuk 2: sedentair gedrag, lichamelijke activiteit en fysiek functioneren}

Beperkingen in fysiek functioneren gaan vaak gepaard met een slechtere kwaliteit van leven en met minder zelfstandigheid. Beperkingen in fysiek functioneren komen vaker voor op hogere leeftijd. Omdat sedentair gedrag ook toeneemt met leeftijd, is het belangrijk om te weten of sedentair gedrag een risicofactor is voor beperkingen in fysiek functioneren. Fysiek functioneren werd bepaald door het meten van spierkracht en loopsnelheid. Meer sedentair gedrag bleek slechts zwak samen te hangen met lagere spierkracht en loopsnelheid. Lichamelijke activiteit, in het bijzonder activiteit van hogere intensiteit, bleek duidelijk gerelateerd aan betere scores op deze testen. Deze resultaten onderstrepen het belang van lichamelijke activiteit om fysieke beperkingen te voorkomen.

\section{Hoofdstuk 3: sedentair gedrag, lichamelijke activiteit en fitheid}

Fitheid is een sterke voorspeller voor gezondheid. Voornamelijk de frequentie van lichamelijke activiteit van hogere intensiteit is bepalend voor iemands fitheid. Of sedentair gedrag gerelateerd is aan een lagere fitheid, onafhankelijk van lichamelijke activiteiten van hogere intensiteit is niet duidelijk. De hoeveelheid sedentair gedrag per dag bleek geassocieerd met een lagere fitheid, onafhankelijk van lichamelijke activiteiten met hogere intensiteit. Verder vonden we dat het (in theorie) vervangen van één uur zitten met één uur lichamelijke activiteit van lage intensiteit samenhangt met een hogere fitheid. Het vervangen van één uur zitten met één uur lichamelijke activiteit van hoge intensiteit hangt samen met een nog hogere fitheid. Eén uur staan in plaats van zitten lijkt niet samen te hangen met een hogere fitheid. Dit betekent dat zitten vervangen voor elke vorm van lichamelijke activiteit al gepaard zou gaan met een betere fitheid. 


\section{Hoofdstuk 4: sedentair gedrag en cardio-metabole gezondheid in NHANES}

In dit hoofdstuk hebben we gekeken of zowel sedentair gedrag, lichamelijke activiteit en fitheid, onafhankelijk van elkaar gerelateerd zijn aan cardio-metabole gezondheid. Hiervoor maakten we gebruik van gegevens van volwassen van 18-49 jaar die deelnamen aan NHANES. Onze resultaten lieten zien dat meer dagelijkse lichamelijke activiteit van hogere intensiteit en een betere fitheid gerelateerd waren aan een beter cardiometabole gezondheid: kleinere buikomvang, minder triglyceriden (vetzuren) in het bloed en een hoger HDL-cholesterol gehalte. Aan de andere kant vonden we in deze populatie geen relatie tussen sedentair gedrag en cardio-metabole gezondheid. Mogelijk was dit het resultaat van een relatief gezonde studiepopulatie.

\section{Hoofdstuk 5: sedentair gedrag en lichamelijke activiteit versus fitheid}

In hoofdstuk 5 hebben we opnieuw onderzocht of sedentair gedrag, lichamelijke activiteit van hogere intensiteit en fitheid gerelateerd zijn aan cardio-metabole gezondheid. Echter deze keer in De Maastricht Studie; dus bij mensen in de leeftijd 40-75 jaar. We vonden dat zowel weinig sedentair gedrag, meer lichamelijke activiteit van hogere intensiteit en een hogere fitheid onafhankelijk van elkaar samenhingen met een lager risico op het metabool syndroom en type 2 diabetes. Van deze relaties bleek fitheid de sterkste samenhang te vertonen. Verder hebben we ook gekeken naar gecombineerde effecten van sedentair gedrag - fitheid en lichamelijke activiteit van hogere intensiteit fitheid. Ook hierin bleek het effect van fitheid het grootste; combinaties met een lage fitheid hingen samen met een verhoogd risico op het metabool syndroom en type 2 diabetes. Ook de combinatie hoge fitheid - veel sedentair gedrag bleek samen te hangen met een hoger risico op deze metabole uitkomsten ten opzichte van de combinatie hoge fitheid - weinig sedentair gedrag. De combinaties lage fitheid - veel zitten en lage fitheid - weinig lichamelijke activiteit van hogere intensiteit hingen het sterkst samen met een hoger risico op het metabool syndroom en type 2 diabetes. Om het risico op cardio-metabole ziektes te minimaliseren lijkt het dus belangrijk om aandacht te besteden aan zowel fitheid, lichamelijke activiteit, als sedentair gedrag.

\section{Hoofdstuk 6: vervangen van sedentair gedrag}

Omdat sedentair samenhangt met een verhoogd risico op hart- en vaatziekten en type 2 diabetes, wilden we in hoofdstuk 6 bestuderen wat er zou gebeuren als men zitten zou vervangen voor staan of lopen. Op basis van statistische modellen konden we berekenen dat 30 minuten zitten vervangen voor 30 minuten lopen al een gunstig effect had op de cardio-metabole gezondheid (bijvoorbeeld lagere vetwaardes in het bloed) en de kans op het hebben van type 2 diabetes. Opvallend was dat het vervangen van zitten door staan ook gepaard ging met een betere cardio-metabole gezondheid en een kleine- 
re kans op het hebben van type 2 diabetes. Zitten vervangen door elke andere activiteit zou dus al kunnen resulteren in een betere cardio-metabole gezondheid.

\section{Conclusies}

De studies in deze thesis lieten duidelijk zien dat lichamelijke activiteit, voornamelijk dat van hogere intensiteit, sterk samenhangt met fysiek functioneren, fysieke fitheid en cardio-metabole gezondheid. Sedentair gedrag (veel zitten) hing ook samen met een slechtere cardio-metabole gezondheid. Deze relatie bleek onafhankelijk van lichamelijke activiteit van hogere intensiteit en fitheid; zelfs fitte mensen lopen gezondheidsrisico's bij veel zitten. Dit laatste zagen we in het bijzonder in De Maastricht Studie. Veel zitten lijkt ook samen te hangen met een lagere fitheid. Hiernaast lieten de resultaten zien dat fysieke fitheid sterk samenhangt met cardio-metabole gezondheid. Om cardiometabole complicaties te voorkomen is het dus belangrijk om naast voldoende lichamelijke activiteit van hogere intensiteit een bepaalde balans te vinden tussen de hoeveelheid sedentair gedrag en overige activiteiten.

Een beperking van de studies in deze thesis is dat deze gebaseerd zijn op crosssectionele analyses. Omdat zowel de voorspellende variabele (bijvoorbeeld sedentair gedrag) en de uitkomst (bijvoorbeeld diabetes) tegelijkertijd zijn gemeten, is het moeilijk om oorzaak-gevolg te onderscheiden. Daarom zouden de resultaten moeten worden bevestigd in studies waarbij gebruik wordt gemaakt van longitudinale data (waarbij de uitkomst in de toekomst wordt bepaald) en in interventiestudies (waarbij bijvoorbeeld één groep mensen verplicht veel moeten zitten en een andere groep juist weinig). Daarnaast zijn er studies nodig die onderzoeken hoeveel sedentair gedrag we moeten vermijden en hoeveel lichamelijke activiteit (en van welke intensiteit) we precies nodig hebben om ziektes zo lang mogelijk uit te stellen. 


\section{Valorisation addendum}

Main responsibilities of Dutch universities include education, conduction of scientific research, and the transition of knowledge to society; valorisation. Knowledge valorisation is defined as the process of creating value from knowledge, by making knowledge suitable or available for social (and economical) use and by making knowledge suitable for translation into products, services, processes and new commercial activities. ${ }^{1}$ This addendum describes the potential benefits of the present thesis on society.

\section{Why are the results from this thesis relevant?}

Currently, our society is faced with an ageing population, in which the prevalence of cardio-metabolic diseases (e.g. cardiovascular disease and type 2 diabetes) and limitations in physical function is increasing. These adverse health conditions not only pose a burden on patients, but also on society due to elevated costs of health care and loss of production. From a public health perspective, delaying the onset or preventing these conditions is desirable.

As described in this thesis, one of the key modifiable determinants of adverse health is physical inactivity. In particular the role of higher intensity physical activity (HPA) has been examined extensively. People who engage more often in HPA have a lower risk of developing cardio-metabolic diseases and functional limitations. In addition, engaging in HPA is related with better cardio-respiratory fitness, which in itself is a strong health predictor. More recently, studies show that also activities of lighter intensity may improve health. In addition to physical activities, the role of sedentary behaviour (any waking behaviour characterized by low energy expenditure) needs to be addressed. An increasing number of studies have associated sedentary behaviour with adverse health conditions. A large amount of daily sedentary behaviour (sitting), independent of the amount of HPA, has been associated with cardio-metabolic diseases and mortality.

However, questions regarding the health effects of sedentary behaviour remain. For instance, is sedentary behaviour associated with lower CRF and lower physical function, independent of HPA? If so, this may provide new strategies to prevent functional limitations. Further, if a person has a high CRF, is sedentary behaviour still a risk for cardio-metabolic health? Possibly, not everyone may benefit from reductions in sedentary behaviour. Furthermore, what is more strongly associated with cardio-metabolic heath: sedentary behaviour, HPA or CRF? Additional insight into sedentary behaviour, HPA, and CRF and their interrelationship as risk factors for cardio-metabolic health may help to expand public health messages and policies aimed at preventing cardiometabolic diseases. 


\section{What can we learn from these results?}

Results in this thesis add to the current literature and show that physical activity, in particular HPA, is an important determinant of cardio-metabolic health (chapters 4-7), CRF (chapter 3) and physical function (chapter 2). Further, results in this thesis showed that sedentary behaviour is associated with cardio-metabolic health and CRF, even when adjusted for HPA (chapters 5 and 6). This implies that reducing the amount of sedentary time could lead to improved cardio-metabolic health.

In addition to daily activities, this thesis focused on CRF as determinant of cardiometabolic health. CRF was even more strongly associated with cardio-metabolic health than HPA (chapter 5). Thus, sub-maximal exercise testing, such as performed in the present thesis, provides important insight to predict future health problems. Therefore, it could be argued that CRF should be incorporated in the evaluation and management of cardio-metabolic disease risk and "low CRF" should be considered as a condition that requires treatment. ${ }^{2}$

\section{For whom may these findings be relevant?}

In a clinical setting, increasing physical activity has been used as an intervention to improve cardio-metabolic health for many years. In addition to physical activity, reducing the amount of daily sedentary time should be regarded as potential intervention to improve health. Results from our studies (chapters 3, 5 and 6) and others have demonstrated that reducing the amount of sedentary time may improve cardio-metabolic health. Thus, physicians should encourage patients to sit less. However, at this time specific recommendations on the amount of sedentary time to decrease are difficult to make. The importance of regular physical activity and sedentary behaviour should also be addressed in many other settings: environmental, school, work.

The population should have easy access to an environment that stimulates physical activity and dissuades from sitting too much. For instance, parks and recreational areas with public sport facilities (football fields, skate parks etc.) may stimulate physical activity. In addition, promoting active transport (e.g. by building bike lanes and foot paths) seems a powerful strategy to stimulate physical activity. ${ }^{3}$ Employers could provide their employees with more options to reduce the amount of sedentary time during working hours. Examples are stand- or cycling work stations and standing or walking meetings. These things have been implemented on a small scale and show promise in reducing the amount of sedentary time during working hours. ${ }^{4}$ Results from chapter 6 indicate that replacing 30 minutes of sedentary time per day with other activities was already associated with improved cardio-metabolic risk markers. In addition to potential health gain, standing and walking during meetings appears to increase efficiency. 
Children and adolescents require more physical activity than adults. Therefore, it is important that they are stimulated to be physically active. For instance, on school days, students should be encouraged to go outside and play during breaks. In addition, more gym classes could be scheduled. It is important to stimulate physical activity during youth/adolescence as physical activity levels at this age may track into adulthood. ${ }^{5}$

Up-to-date, science-based physical activity guidelines, such as recently proposed by the Dutch health council (Gezondheidsraad), are needed to stimulate physical activity in all the settings described above. These

\section{What's next?}

The results from this thesis may stimulate new research projects. As mentioned in the general discussion, results from this thesis are limited by the cross-sectional analyses; sedentary behaviour, physical inactivity and low CRF may (partly) be the product of poor health. Therefore, causality should be determined in longitudinal studies. Longitudinal data on sedentary behaviour and mortality is available for NHANES, but the period of follow-up is relatively short. ${ }^{6}$ In particular, longitudinal results from The Maastricht Study may provide valuable insight into the causality of sedentary behaviour as sedentary behaviour is accurately captured by the posture based activity monitor.

Second, dose-response effects should be studied. This can be done in an observational setting, but also in an experimental setting. These studies should explicate how much sitting is too much and how much physical activity do we need in order to remain in good health? Do we need to engage in physical activity throughout the day, or are a few longer bouts of physical activity per week sufficient? At what intensity should this physical activity be performed? Should we really avoid long bouts of uninterrupted sitting? This type of information is crucial to develop targets for interventions. In chapter 6 we demonstrated that theoretically replacing 30 minutes of sedentary time with standing was already associated with lower risk of having the metabolic syndrome and T2DM. However, will similar effects also be observed in an experimental setting? Further, results from chapter 5 suggest that a shift from low CRF to medium CRF is associated with greatest reduction in the likelihood of having the metabolic syndrome and type 2 diabetes. An increase in CRF may already be achieved by substituting sedentary time for light intensity physical activities (chapter 3), but will this indeed result in markedly improved CRF?

Finally, we must examine the feasibility of changes in daily activities and CRF. What amount of sedentary time can be reduced and how much can physical activity be increased? Importantly, can improvements in the balance of daily activities be sustained 
over a longer period of time? Consumer-based wearable activity monitors (such as the Fitbit and Jawbone) and applications on smartphones, may aid individuals in sustaining an acquired healthy activity pattern.

All this (future) work will contribute to a better understanding of the health consequences of physical activity and sedentary behaviour. Physical activity guidelines, such as implemented by the WHO may require updates when our knowledge in this field progresses.

\section{References}

1. VSNU Vereniging van universiteiten. Een Raamwerk Valorisatie-indicatoren. Van Deventer, 'sGravenzande 2013. Available from:

http://www.vsnu.nl/files/documenten/Domeinen/Onderzoek/Valorisatie/130422\%20\%20VSNU\%20Raamwerk\%20Valorisatie-indicatoren_web.pdf.

2. Després J-P. Physical Activity, Sedentary Behaviours, and Cardiovascular Health: When Will Cardiorespiratory Fitness Become a Vital Sign? Can. J. Cardiol. 2016;32(4):505-513.

3. Goenka S, Andersen LB. Urban design and transport to promote healthy lives. The Lancet 2016;388(10062):2851-2853.

4. Neuhaus M, Eakin EG, Straker L, Owen N, Dunstan DW, Reid N, Healy GN. Reducing occupational sedentary time: a systematic review and meta-analysis of evidence on activitypermissive workstations. Obes. Rev. 2014;15(10):822-838.

5. Telema R, Yang X, Viikari J, Valimaki I, Wanne O, Raitakari O. Physical activity from childhood to adulthood a 21-year tracking study. Am. J. Prev. Med. 2005;28(3):267-73.

6. Koster A, Caserotti P, Patel KV, Matthews CE, Berrigan D, Van Domelen DR, Brychta RJ, Chen KY, Harris TB. Association of sedentary time with mortality independent of moderate to vigorous physical activity. PLoS ONE 2012;7(6):e37696. 


\section{Dankwoord}

Hier ligt het dan, mijn proefschrift. Na vier jaar, toch wel een opluchting. Al staat mijn naam op de voorkant, dit proefschrift zou er nooit geweest zijn zonder de hulp van velen. Ik wil dan ook allen (collega's, coauteurs, vrienden, familie) bedanken die hieraan een bijdrage hebben geleverd. Graag maak ik van deze gelegenheid gebruik om een aantal mensen in het bijzonder te bedanken.

Mijn promotie team: Nicolaas, Hans en Annemarie. Bedankt voor jullie vertrouwen en geduld. Ik heb me vanaf het begin erg prettig gevoeld met jullie als begeleiders. Ik kijk terug op een erg fijne samenwerking, waarbij ik altijd het gevoel heb gehad dat ik bij jullie terecht kon wanneer ik dat nodig achtte. Ik waardeer het zeer dat jullie mij de mogelijkheid boden om verschillende epidemiologische cursussen te volgen en congressen te bezoeken.

Nicolaas, bedankt voor al je kennis en het klinische perspectief van waaruit jij onze discussies benaderde. Veel dank ook voor de tijd je hebt genomen om vele versies van papers en abstracts door te nemen en te voorzien van commentaar.

Hans, ook jij bedankt voor alle bewegingswetenschappelijke kennis en ervaring die je met me hebt gedeeld. Ik waardeer je getailleerde feedback op alle papers, abstracts en presentaties.

Annemarie, wat fijn dat jij mijn dagelijkse begeleider was! Bedankt dat ik altijd bij je binnen mocht lopen. Ik heb veel van je geleerd op epidemiologisch gebied en ik waardeer al je hulp en tijdige commentaar op elk stuk wat ik je ooit heb gestuurd.

De leden van mijn beoordelingscommissie, prof. dr. ir. M.P. Weijenberg (voorzitter), dr. J.B.J. Bussmann (Erasmus universitair medisch centrum, Rotterdam), prof. dr. M.K.C. Hesselink, prof. dr. J.P. Kooman en prof. dr. ir. M. Visser (VU medisch centrum, Amsterdam). Bedankt voor de tijd en aandacht die jullie hebben besteed aan de beoordeling van mijn proefschrift.

Collega's van De Maastricht Studie. Gedurende alle jaren die ik bij DMS heb doorgebracht heb ik veel mensen zien komen en gaan. Bijna te veel om op te noemen, dus ik doe het zo: allen, bedankt voor de fijne samenwerking bij V1, V2 en zelfs een tijdje bij V3! Bedankt ook voor de gezellige momenten buiten DMS, bijvoorbeeld bij Thembi, de donderdagmiddagborrels, het ambtenaren carnaval, spelavonden en het dms-aioweekendje. Van deze club wil ik één iemand nog wel noemen...

Juul, de eerste "activPAL aio". Al zijn wij op papier heel verschillend, in de praktijk bleek het goed te werken. Bedankt voor al je hulp. Ook bedankt voor je gezelligheid, zowel in Maastricht als tijdens congressen en cursussen, voor alle nuttige (en zeker ook 
de minder nuttige discussies) die we hebben gevoerd en de wedstrijdjes die we hebben gehouden.

Zonder ontspanning geen inspanning. Alle vrienden en vriendinnen van Fuego, Ormètikos/Otrias, Neuf, de wooden spoons en skrups en co. Bedankt voor avonden en weekenden vol gekkigheid en gezelligheid. Ik hoop dat er nog vele zullen volgen!

Lieve pap, mam, Mart, broertjes, zusjes. Dank voor jullie liefde die jullie mij al jaren geven. In Breda of Sassay, ik vind het altijd fijn om 'thuis' te komen. Ik hou van jullie! Ook alle Leerinks, veel dank voor jullie warmte en gezelschap.

Lieve Wendy. Wat vind ik het fijn om met jou samen te zijn. Bedankt voor al je liefde, vrolijkheid en steun. Ik hou van je! 


\section{Curriculum Vitae}

Jeroen van der Velde was born on January 8th, 1986 in Breda, the Netherlands. After graduating from secondary school (Stedelijk Gymnasium, Breda) in 2004 he moved to Maastricht and started the Bachelor of Health Sciences at Maastricht University. After graduation, he finished the Master of Physical Activity and Health, also at Maastricht University. As part of his master program, Jeroen completed a research internship at the University of Wollongong, Australia. In 2011, he became involved in The Maastricht Study as a research assistant. In 2013, he started the PhD-project of which the results are described in the present thesis. This project, embedded within The Maastricht Study, was supervised by prof. Nicolaas Schaper, prof. Hans Savelberg and dr. Annemarie Koster. Currently, Jeroen is appointed as postdoctoral researcher at Maastricht University. 
